\title{
IMAGE MATCHING AND IMAGE SUPER-RESOLUTION VIA DEEP LEARNING
}

\author{
A Dissertation presented to \\ the Faculty of the Graduate School \\ at the University of Missouri
}

In Partial Fulfillment

of the Requirements for the Degree

Doctor of Philosophy

by

GIANG D. BUI

Dr. YE DUAN, Desertation Supervisor

MAY 2018 
The undersigned, appointed by the Dean of the Graduate School, have examined the dissertation entitled:

\section{IMAGE MATCHING AND IMAGE SUPER-RESOLUTION VIA DEEP LEARNING}

presented by Giang Bui, a candidate for the degree of Doctor of Philosophy and hereby certify that, in their opinion, it is worthy of acceptance.

Dr. YE DUAN

Dr. Committee Member

Dr. Committee Member

Dr. Committee Member

Dr. Committee Member 


\section{ACKNOWLEDGMENTS}

First of all, I am most grateful to my supervisor, Prof. Ye Duan, not only for his guidance, but especially for his outstanding support - both with difficult academic problems and in daily life. He has walked me through all the stages of the writing of this thesis. Without his consistent and illuminating instruction, the completion of this thesis would not have been possible. I am very grateful for the humane environment the Computer Vision and Image Understand lab has provided during the years of my study in Mizzou.

I would also like to acknowledge and thank the faculty members and other students who contributioned to this work and advised me during graduate school including Dr. Prasad Calyam, Jeffrey Uhlmann, Jialin Cheng, Zhihai He, Brittany Morago, Truc Le

Lastly, I would like to thank all my friends and family for their support, enocuragement, and laughs as I completed my Ph.D. including Van Nguyen, Bao Bui, Lam Bui, Tho Bui, Linh Bui, Hue Nguyen, Tien Nguyen, Thuy Nguyen, Anh Chu. 
TABLE OF CONTENTS

ACKNOWLEDGMENTS ................ ii

LIST OF TABLES $\ldots \ldots \ldots \ldots \ldots \ldots$ vii

LIST OF FIGURES $\ldots \ldots \ldots \ldots \ldots \ldots \ldots \ldots$ ix

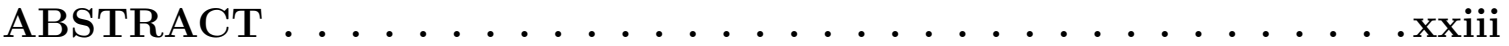

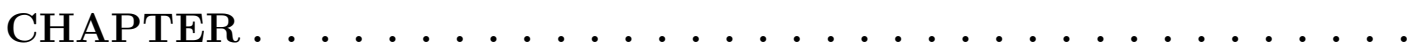

1 Introduction ......................... 1

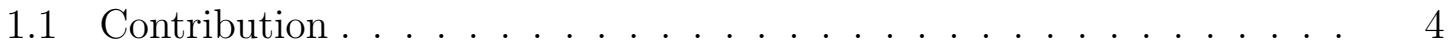

1.2 Organization $\ldots \ldots \ldots \ldots \ldots \ldots \ldots \ldots \ldots \ldots \ldots \ldots$

2 An Ensemble Approach to Image Matching Using Contextual Features ...................... 8

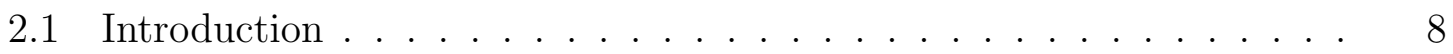

2.1 .1 Background . . . . . . . . . . . . . . . . . . 10

2.1.2 Overview and Contributions . . . . . . . . . . . . . . . 14

2.1 .3 Notation . . . . . . . . . . . . . . . . . . . . . . . . 17

2.2 Contextual Feature Matching . . . . . . . . . . . . . . 17

2.2 .1 Adaptive MSER Neighborhoods . . . . . . . . . . . . . . 18

2.2 .2 Contextual Line Segments . . . . . . . . . . . . . . . . 20

2.2 .3 Contextual HOG . . . . . . . . . . . . . . . . . . . . . 21

2.2 .4 Ensemble SIFT . . . . . . . . . . . . . . . . . . . . . . 23

2.2.5 Contextual Harris-Laplacian . . . . . . . . . . . . . . 25

2.2 .6 Identifying Pseudo Corners . . . . . . . . . . . . . . 26

2.3 Homography Estimation, Refinement, and Verification _ . . . . 27 
2.4 Results and Discussion . . . . . . . . . . . . . . . . . 30

3 2D Matching Using Repetitive and Salient Features in Architectural Images . . . . . . . . . . . . . . . . . . 41

3.1 Literature Review . . . . . . . . . . . . . . . . . . . . . . . . . 41

3.2 Contributions . . . . . . . . . . . . . . . . . . . 43

3.3 Two-Step Approach . . . . . . . . . . . . . . . . . . . . . . . . . . . . 44

3.3 .1 Vertical Alignment . . . . . . . . . . . . . . . . . 45

3.3.2 Horizontal Alignment . . . . . . . . . . . . . . . . . 46

3.4 Evaluation and Discussion . . . . . . . . . . . . . . . 51

3.4 .1 Experimental Setup . . . . . . . . . . . . . . . . . . 51

3.4 .2 Results . . . . . . . . . . . . . . . . . . . . . 52

4 Image Super-Resolution with Deeply Supervised Nets and Incremental Learning . . . . . . . . . . . . . 66

4.1 Introduction . . . . . . . . . . . . . . . 66

4.2 Related Works . . . . . . . . . . . . . . . . . . . . 67

4.2 .1 Non-deep learning methods . . . . . . . . . . . . . . 67

4.2.2 Deep-learning methods . . . . . . . . . . . . . . . . . . . . . 69

4.2 .3 Deep-learning methods . . . . . . . . . . . . . . . . . . 70

4.3 Deeply Supervised Nets with Incremental Learning for Image SuperResolution . . . . . . . . . . . . . . . . . . . . . . . 71

4.3.1 Mathematical formulation . . . . . . . . . . . . . . . 71

4.3 .2 Network architecture . . . . . . . . . . . . . . . . . . . 73

4.3 .3 Incremental Learning with DSN . . . . . . . . . . . . . . 74

4.4 Experiments . . . . . . . . . . . . . . . . . 75

4.4 .1 Datasets . . . . . . . . . . . . . . . 76

4.4 .2 Implementation details . . . . . . . . . . . . . . . 76 
4.4.3 Investigation of Different Settings . . . . . . . . . . . 77

4.4.4 Single Model for Multiple Scales . . . . . . . . . . . . . 78

4.4.5 Comparisons with State-of-the-Art Methods . . . . . . . . 81

4.5 Conclusion . . . . . . . . . . . . . . . . . . . . . 81

4.6 Limitation and Future Work . . . . . . . . . . . . . . . . . . 81

5 Point Based Rendering Enhancement via Deep Learning _ . . . 86

5.1 Overview . . . . . . . . . . . . . . . . . 86

5.2 Related Works. . . . . . . . . . . . . . . . . . . 87

5.3 Proposed Method . . . . . . . . . . . . . . . . . 90

$5.3 .1 \quad$ Input data . . . . . . . . . . . . . . . . . . 91

$5.3 .2 \quad$ Splat rendering . . . . . . . . . . . . . . . . . . . 91

5.3.3 Deep-Learning based Super-Resolution with Generative Adversarial Network . . . . . . . . . . . . . . . 93

5.4 Experiments . . . . . . . . . . . . . . . . . . . . . 97

5.4 .1 Training Dataset . . . . . . . . . . . . . . 97

5.4 .2 Implementation details . . . . . . . . . . . . . . . . 98

5.4.3 Comparison with state-of-the-art rendering techniques $\ldots .99$

5.4.4 Interactive 3D Scene Navigation . . . . . . . . . . . . 101

5.4 .5 Image matching . . . . . . . . . . . . . . . . . . 102

6 Summary and concluding remarks . . . . . . . . . . . 104

6.1 Future work . . . . . . . . . . . . . . . . . . . . . 104

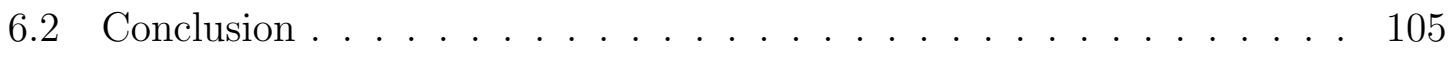

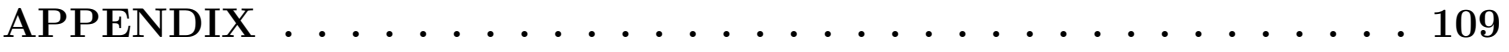

A Supplementary Material for An Ensemble Approach to Image Matching Using Contextual Features . . . . . . . . . . . . . 109 
A.1 Comparison with Other Methods . . . . . . . . . . . . . . . . 110

A.2 True Positive/False Positive Rates . . . . . . . . . . . . . . . . . . . . 111

A.3 Complexity . . . . . . . . . . . . . . . . 116

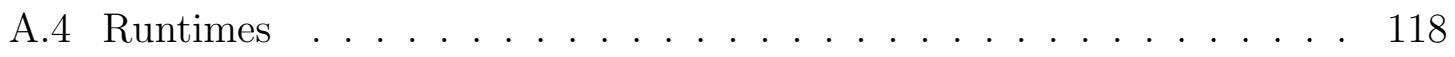

A.5 Supplemental Quantitative Registration Results . . . . . . . . . . . . 121

B Supplementary Material for 2D Matching Using Repetitive and Salient Features in Architectural Images . . . . . . . . . . . 124

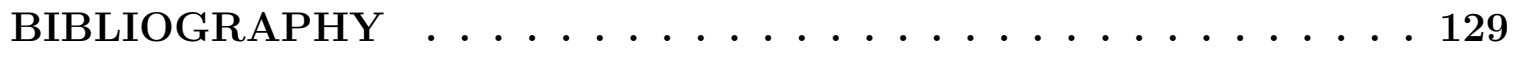

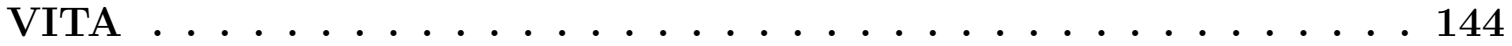




\section{LIST OF TABLES}

Table $\quad$ Page

2.1 Average Precision on Symmetry Dataset for Varying Graph Matching Parameters - dissimilarity score and threshold on required \% of matching lines . . . . . . . . . . . . . . . . . . 21

3.1 Repeatability Comparison on ZuBuD Dataset . . . . . . . . . . 56

3.2 Average Runtimes in Seconds and Number of Features for Stages of Our Pipeline on ZuBuD Dataset . . . . . . . . . . . . . . . 57

4.1 Incremental Learning with $M=4 \ldots \ldots$. . . . . . . . 76

4.2 Benchmark results. Average PSNR/SSIMs for scale factor $\times 2, \times 3$ and $\times 4$ on datasets Set5, Set14, B100 and Urban100. Red color indicates the best performance and blue color refers the second best . . . . . . 77

4.3 PSNR on Set14(×4) with different network configurations . . . . . . 80

4.4 PSNR on Set14(×4) with scale augmentations. Red color indicates the best performance and blue color refers the second best. . . . . . . . . 80

5.1 Quantitative comparison of the approaches on the testing sets. . . . . 100

5.2 Running time of the proposed method with different image sizes . . . 101

5.3 Rendering time . . . . . . . . . . . . . . . . . . 102

5.4 Feature extraction evaluation . . . . . . . . . . . . . . 103 
A.1 True Positive/True Negative Rates of Ensemble Methods on Stanford Mobile Visual Search Dataset . . . . . . . . . . . . . . . . . 112

A.2 True Positive/False Positive Rates of Ensemble Methods on Landmark Dataset . . . . . . . . . . . . . . . . . . . . 114

A.3 Complexity of Different Stages of Our Pipeline . . . . . . . . . . 117

A.4 Average Run-times in Seconds and Number of Features for Different Stages of Our Pipeline on Symmetry Dataset . . . . . . . . . . . 119

A.5 Average Runtimes in Seconds and Number of Features for Other Methods on Symmetry Dataset . . . . . . . . . . . . . . . . . . 119 


\section{LIST OF FIGURES}

Figure

2.1 Outline of our registration pipeline. Keypoint features are first extracted. SIFT keypoints, Harris-Laplacian corners, or our proposed Pseudo-Corners can be used. If SIFT is used, keypoints are matched using the SIFT descriptor. We then search for matches using contextual features. $I_{1}$ is segmented into MSER-based neighborhoods which are used to determine neighborhood sizes for studying line segments and HOG's. (HOG information in top right image is colored according to the dominant gradient direction for each cell.) Linear and HOG information is used to verify new matches. An aligning homography is calculated and refined using ICP and gradient-based methods. . . . .

2.2 Registration results after applying our pipeline to several challenging image pairs from our dataset as well as from the datasets provided in [1] and [2]. Original images are shown above registered images. . . 
2.3 Mapping bounding box of MSER neighborhood in $I_{1}$ to $I_{2}$. Top: MSER segments and MSER defined neighborhood in $I_{1}$. MSER segment being used is shown in white. Bottom: Original MSER neighborhood in $I_{2}$ and $I_{2}$ 's patch after it has been transformed to the same coordinate system as $I_{1}$ 's patch via a scaling and rotation. The patch in $I_{2}$ is extracted at multiple scales and each patch is rotated such that its $\mathrm{x}$-axis points along the dominant gradient direction in the patch. .

2.4 MSER's extracted at different levels of a Gaussian pyramid. Segments are combined from different levels to create adaptive MSER segments shown in Figure 2.1. . . . . . . . . . . . . . . . . .

2.5 Matching lines in the neighborhood of a feature point. Left: Line segments are identified within neighborhoods centering a potential match. The keypoint is shown in black in both neighborhoods and the relative scales are represented by black circles. Middle: Line segments are converted to polar coordinates based on their distance from the keypoint and their orientation in relation to neighborhood's dominant gradient direction. Right: Hungarian graph matching is applied to the set of polar coordinates to figure out the optimal set of matching lines. . . .

2.6 Identifying matching neighborhoods for contextual matching. This example uses a SIFT match as an anchor for exploring contextual neighborhoods. Left: Two images are matched and share a corresponding point, shown in yellow. This keypoint pair has a distinctiveness ratio between 0.7 and 0.9 and is not verified by SIFT. Top Right: Matching line segments within neighborhood. Bottom Right: HOG descriptors for cells in the neighborhood. Cells are colored according to dominant gradient direction. The line orientation in each cell shows the cell's dominant gradient direction. . . . . . . . . . . . . . . . . 
2.7 Outline of how we use the distinctiveness ratio test to collect matches and to determine when to use contextual matching in conjunction with the SIFT matching scheme. . . . . . . . . . . . . .

2.8 Image alignment improvement using our ensemble SIFT method. Left: Image pair to be aligned. Center: Image alignment using only SIFT features. Right: Image alignment using our ensemble features. . . . . 25

2.9 Using Pseudo Corners to align a difficult image pair, called Vatican. Top: The majority of the SIFT (green) and linearly-verified (red) keypoint matches for this pair are incorrect and cannot be used to align the images. No SIFT+HOG matches were identified in this example. The relative SIFT scales are represented by the size of the circles and the features' orientations are denoted by the black lines. 2nd Row: Line segments extracted from images (shown in yellow) that are used to find Pseudo Corners. 3rd Row: Pseudo Corners identified after intersecting line segments. Bottom: Image pair is aligned using the homography calculated from the matched Pseudo Corners. . . . . . . . . . . .

2.10 Matches identified using multiple homographies. Each color corresponds to a different homography. We can see how each homography tends to find matching points in different regions and planes of the image. 36

2.11 Using gradient information to refine homography. Top: Two original images and their edge images and gradient field images. We aim to align the edge and gradient field images as well as possible. Bottom Left: Stitching result before our refinement method is applied. Bottom right: Result after our refinement is applied. Bottom middle: Zoomed in views of the area where the alignment is corrected through refinement. 36 
2.12 Precision-recall curves for four different image pairs from [2]. Curves are provided for using SIFT matches, our ensemble feature, and just the contextually verified SIFT features identified using our method. Data points correspond to increasing distinctiveness ratios moving from left

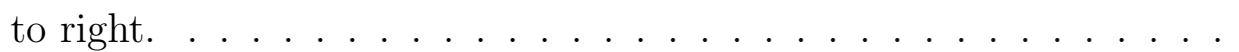

2.13 Quantitative results for stitching image pairs. Top: Percentage of pixels in image plane that are correctly aligned after using solved homography. Center: Percentage of matches that are identified using different methods that are correct. Bottom: Number of matches identified using several different methods. . . . . . . . . . . . . . .

2.14 Repeatability measurements for SIFT, Harris-Laplacian, and Pseudo Corners on Oxford Affine Covariant Region Detectors Dataset [3]. The sets of images test the following changes in image properties: bark scale and image rotation changes on textured scene, bikes - increased blur on a structured scene, boat - scale changes, graf - viewpoint changes, leuven - illumination changes, trees - increased blur on a structured scene, ubc - changes in JPEG compression, wall - viewpoint angle changes on textured scene. . . . . . . . . . . . . . . 
2.15 Comparing our contextual methods. Using the symmetry dataset [2], we show the alignment accuracy using our ensemble SIFT features before and after refinement, contextual Harris-Laplacian before and after refinement, and contextual pseudo corners before and after refinement. As mentioned in Section 2.1.3, ensemble features refer to the version of our method that uses an existing keypoint descriptor, such as SIFT, in addition to our contextual linear and contextual HOG information for matching. Contextual features use only our contextual descriptor for matching. The accuracy is measured in terms of the percentage of pixels in $I_{1}$ that are transformed to the correct location in $I_{2}$ using the homography estimated from the match set. . . . . . . . . . . . .

3.1 Pipeline of our proposed registration method. Our method has two main stages: vertical alignment and horizontal alignment. For vertical alignment, we perform dimension reduction, identify representative patches for each image, and use the matching representatives to compute a 1-D transformation that transforms the images to the same scale and aligns the pair along the vertical axis. For horizontal alignment, we compute the intra-image saliency and search for strong, salient matches along corresponding rows between images to vote for the correct displacement between the pair. $\ldots \ldots \ldots \ldots \ldots \ldots$ 
3.2 Identifying groups of repetitive elements and matching groups. (a.) Similar patches are identified along a row of an image. Teal boxes surrounding a larger dot show the matching anchor keypoints. Yellow boxes surrounding a smaller dot show the dense features that were matched to the anchor keypoint for the row. For this example, we use Pseudo Corners as anchor keypoints as is discussed in Section 3.4.2. (b.) The chosen representative patch of the group. This patch is most similar to the average of all the elements contained in the group. Note that sometimes in architectural designs, the repetitive elements may not occur at regular intervals as shown in this example. Our method is designed to search for repetition regardless of the regularity or irregularity of the interval. (c.) Subset of the corresponding groups after computing a 1-D transformation are highlighted in matching colors. Every element of the groups are displayed. . . . . . . . . . . . .

3.3 Motivation for searching for salient matches for horizontally aligning images. The highlighted image patches in the above image pair shows a set of matching repetitive elements. If we choose the horizontal alignment that maximizes the number of overlapping elements, we will have the wrong image pair transformation in situations like this where the images do not fully overlap. The highlighted patches are colored according to which patches will match if we try to maximize the number of overlapping patches. This is why we use our reduced search space (after vertical alignment) to identify distinct areas and salient matches to choose potential horizontal alignments. . . . . . . . . . . . 
3.4 Visualization of match saliency equation. (a.) Original images being matched. (b.) Intra-image saliency map of each image. More salient areas appear in red and more repetitive areas appear in blue. (c.) Color fields of match saliency scores shown from the perspective of both images. (d.) Result after applying Otsu thresholding. All keypoint matches that are contained within the remaining colored regions are used to vote for a hortizontal alignment. . . . . . . . . . . . .

3.5 Accuracy measures for aligning images vertically, which is the first stage of our two-step pipeline. We show the results on our three datasets of using the traditional SIFT method, only the y-coordinates of the SIFT matches (1-D SIFT), and using our proposed method for vertical alignment (1-D Grouping). . . . . . . . . . . . .

3.6 Accuracy measures for aligning images both vertically and horizontally. We show the results on our three datasets of using the traditional SIFT method, using SIFT matches to align the images first vertically, then horizontally (SIFT Two-Step), our proposed method for vertical then horizontal alignment (Grouping Two-Step), and our full pipeline. . .

3.7 Accuracy measures for fully aligning images using our complete pipeline compared to state-of-the-art methods. We show the results on our three datasets of using our proposed method (Repetition), our proposed method with refinement (Refined), the traditional SIFT method, the contextual method described in [4], the two vartions of the symmetrybased features method described in $[2] \ldots$. . . . . . . . . . . . . 62

3.8 Precision measurements of matches found in the keypoint matching methods tested and shown in Figure 3.8. We show the results on our three datasets of using our proposed method (Repetition), the traditional SIFT method, and the contextual method described in [4]. 
3.9 The number of matches found with the keypoint matching methods tested and shown in Figures 3.8 and 3.7. . . . . . . . . . . . .

3.10 Visual alignment results. The original image pair is shown above each of the checkerboard views of the aligned result. These image pairs poses a variety of challenges to matching in addition to being repetitive such as different rendering styles, time changes, partial facade overlap, scale changes, and lighting changes. Image pairs shown are from datasets provided by $[2],[5]$, and $[6] \ldots \ldots \ldots \ldots$

3.11 Alignment of images containing multiple planes. (a.) Original images, before and after rectification, that are being matched. (b.) Subset of the corresponding repetitive groups after computing a 1-D transformation (Section 3.3.1) are highlighted in matching colors. (c.) Images shown after they have been vertically aligned. (d.) Intra-image saliency map of each image. The four color fields for each image show the innerimage saliency computed at different scales. More salient areas appear in red and more repetitive areas appear in blue. (e.) Color fields of match saliency scores shown from the perspective of both images. (f.) Result after applying Otsu thresholding to match saliency score images. (g.) Displacement maps. Each match visualized in (f.) votes for an alignment. (h.) Result of aligning the image pair using the two different horizontal displacements chosen in (e.) The left result aligns the salient tree that is in front of (and on a different plane than) the building of interest. The right result uses the salient matches on the building facade to correctly align the region of interest. . . . . . . 
4.1 Our proposed network architecture: (Top) Multiple network blocks with deep supervision, every intermediate result is penalized by a loss function $l$. (Bottom) The layout of each network block with a corresponding number of feature maps followed by kernel size indicated for each convolutional (e.g. 64@3x3 stands for 64 3x3 kernels). Our final model consists of four blocks $(M=4)$ and 20 layers $(d=20)$ within

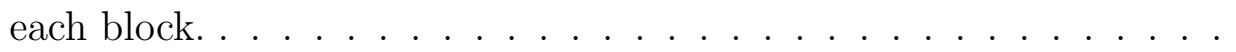

4.2 A simple stacked model with four network blocks. . . . . . . . . . . . 72

4.3 Evaluations performed on Set14 dataset. (a): Number of blocks versus accuracy for the scale factor $\times 4$. More blocks mean deeper network which leads to better accuracy. (b): Number of block's layers versus accuracy for the scale factor $\times 4$. More layers within a block yield deeper network which leads to better accuracy. (c): Learning curve of various SRDSN at different configurations. We fix the number of the convolutional layer for each block and change the number of blocks. More blocks yield better accuracy. (d) Learning curve of SRDSN, SRDSN w/o incremental learning, and stacked model. Both SRDSN w/o incremental learning and stacked model flat out very quickly while SRDSN improves significantly after stage training transitions. . . .

4.4 Comparisons with other methods $(\times 3)$. Each image row is followed by a zoom-in image row marked with red rectangles. (1) Super-resolution results of "img059" (Urban100) with scale factor x3. SRDSN recovers sharp lines. (2) The second example shows image "ppt3" (Set14 with scale factor $\mathrm{x} 3$ ). Texts in SRDSN are sharp, while others are blurry. (3) The third row shows image "baboon" (Set15 with scale factor x3). SRDSN recovers high frequency regions, while others all give blurry results. . . . . . . . . . . . . . . . . 
4.5 Comparisons with other methods $(\times 2)$. Each image row is followed by a zoom-in image row marked with red rectangles: (1) Super-resolution results of "img015" (Urban100) with scale factor x2. SRDSN recovers sharp lines. (2) The second example shows image "img083" (Urban100 with scale factor $\mathrm{x} 2$ ). SRDSN recovers the pattern. . . . . . . .

4.6 Comparisons with other methods $(\times 4)$. Each image row is followed by a zoom-in image row marked with red rectangles: (1) Super-resolution results of "ppt3" (Set14) with scale factor x4. SRDSN recovers sharper text. (2) The second example shows image "comic" (Set14 with scale factor $\mathrm{x} 4$ ) SRDSN recovers high-frequency regions, while others all give blurry results. . . . . . . . . . . . . . . . . .

5.1 Different rendering techniques: (1) Photograph. (2) Splat rendering. (3) Intensity completion. (4) Gradient completion. (5) Texture splat rendering. (6) (Ours) Deep-Learning based rendering with splat rendering of $(2)$ as input. . . . . . . . . . . . . . . . . . . 90

5.2 Proposed method pipeline. . . . . . . . . . . . . . . . . 91

5.3 (Top) Generator Network Structure. The network consists of multiple blocks with deep supervision, every intermediate result is penalized by a Euclidean loss function $l$. The layout of each network block has a corresponding number of feature maps followed by kernel size indicated for each convolutional (e.g. 64@3x3 stands for 64 3x3 kernels). Our final network consists of four blocks $(M=4)$ and 20 layers $(d=20)$ within each block. (Bottom) Discriminator Network Structure with corresponding number of feature maps (n) and stride (s). The network takes a set of natural images to measure the similarity of the generator output image and ground truth image. . . . . . . . . . . . . 
5.4 Comparisons with other methods on synthetic testing set (Set1) for the three examples. From left to right and top to bottom: OpenGL Point Rendering, Texture Mesh, Intensity Completion, Gradient Completion, Splat Rendering, Texture Splat, SRDSN, and SRDSN-GAN. . . . . . 100

5.5 Comparisons with other methods on real testing set (Set2) for the three examples. From left to right and top to bottom: OpenGL Point Rendering, Photograph, Intensity Completion, Gradient Completion, Splat Rendering, Texture Splat, SRDSN, and SRDSN-GAN. . . . . . 101

A.1 Registration results after applying our pipeline to several public dataset image pairs presenting challenges including small image overlap, camera rotation, lighting changes, and .JPEG compression. Images provided courtesy of [3], [7], [1] and [2] . . . . . . . . . . . . . . . . . . 110

A.2 Keypoint matches for images from Stanford Mobile Visual Search dataset [8]

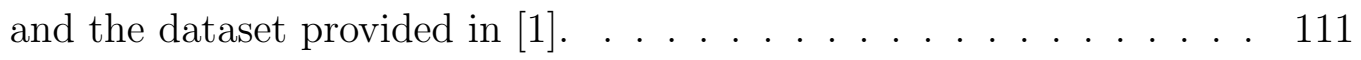

A.3 Registration results after applying our pipeline to several symmetry database pairs. Images provided courtesy of [2]. Top: worldbuilding, Bottom: paintedladies18. From left to right: The original image pair, SIFT Flow results, stitched images using the SIFT+Symmetry method described in [2], and stitched images using our pipeline. For the SIFT Flow results, we show the colored pixel displacement map on the left and the $I_{2}$ warped to $I_{1}$ 's image plane on the right. . . . . . . . . . . 112 
A.4 Comparing our ensemble methods to other matching techniques. We show in this chart accuracy measures for aligned image pairs using Ensemble SIFT-based features, Contextual Harris Laplacian features, Contextual Pseudo Corners, MSER with SIFT descriptors, SIFT Flow [9], and Local Symmetry Features [2]. For the Local Symmetry Features method, we tested using just the symmetry descriptor and the symmetry descriptor concatenated with each feature point's SIFT descriptor as described in $[2] . \ldots \ldots \ldots \ldots \ldots$

A.5 Image pairs from Landmark dataset that are used for True Positive/False Positive tests in Table A.2. . . . . . . . . . . . . . . 115

A.6 Image pair alignment accuracy and number of matches found using Contextual Harris-Laplacian and Contextual Pseudo Corners on various datasets. The results for Figure 13 correspond to the images shown in Figure 13 in the submitted paper from left to right and come from the dataset provided by [1]. The results for Figure A.2 correspond to the images in Figure A.2 in this document from left to right and from top to bottom. These images come from the datasets provided by [1]

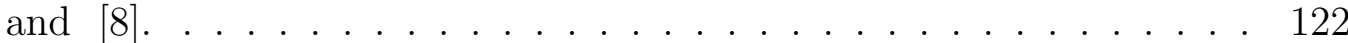

A.7 Image pair alignment accuracy and number of matches found using Contextual Harris-Laplacian and Contextual Pseudo Corners on images from the png-ZuBuD dataset [6]. Images object0001-view1:2 through object0029-view1:5 are images of buildings in Zurich from various view points. Each building is assigned an object number and there are five views of each building. We matched view 1 to view 2 , view 1 to view 3, and so for each building set for objects 1-10 and 21-29 in this dataset. 123 
B.1 Test of our proposed dimension reduction on the $\mathrm{ZuBuD}$ dataset. Here we compare the 1-dimensional accuracy (along the y-axis) of different methods. The first is to using traditional SIFT matches with the 8point algorithm to compute a full 8 degree of freedom homography. The second approach is to use traditional SIFT matches, but compute a 1-D, 2 degree of freedom transformation using just the y-coordinates of the SIFT matches. The third approach is the grouping method described in this paper to compute a 1-D vertical transformation . . . 125

B.2 Test of our proposed dimension reduction. Here we compare the 1dimensional accuracy (along the y-axis) of different methods. The first is to using traditional SIFT matches with the 8-point algorithm to compute a full 8 degree of freedom homography. The second approach is to use traditional SIFT matches, but compute a 1-D, 2 degree of freedom transformation (as described in the main paper) using just the y-coordinates of the SIFT matches. The third approach is the grouping method described in this paper to compute a 1-D vertical transformation. . . . . . . . . . . . . . . . 125

B.3 Test of our proposed dimension reduction. Here we compare the 1dimensional accuracy (along the y-axis) of different methods. The first is to using traditional SIFT matches with the 8-point algorithm to compute a full 8 degree of freedom homography. The second approach is to use traditional SIFT matches, but compute a 1-D, 2 degree of freedom transformation (as described in the main paper) using just the y-coordinates of the SIFT matches. The third approach is the grouping method described in this paper to compute a 1-D vertical transformation. ......................... 126 
B.4 Test of our proposed two-step on the $\mathrm{ZuBuD}$ dataset. As a baseline, we show the accuracy of using traditional SIFT matching to compute a standard 8 DOF homography (Traditional SIFT). We then show tests for using 1) SIFT features (SIFT Two-Step) and 2) grouping features (Grouping Two-Step) to compute a 1-D vertical transformation. For both of these tests, we using matched SIFT features along corresponding rows to vote for a horizontal 1-D transformation. To show the strength of our full pipeline, we have also included in this chart the results of using the two-step approach with the intra-image saliency and pairwise match saliency computations (Full Pipeline). . . . . . . 127

B.5 Test of our proposed two-step on the SymUrban dataset. As a baseline, we show the accuracy of using traditional SIFT matching to compute a standard 8 DOF homography (Traditional SIFT). We then show tests for using 1) SIFT features (SIFT Two-Step) and 2) grouping features (Grouping Two-Step) to compute a 1-D vertical transformation. For both of these tests, we using matched SIFT features along corresponding rows to vote for a horizontal 1-D transformation. To show the strength of our full pipeline, we have also included in this chart the results of using the two-step approach with the intra-image saliency and pair-wise match saliency computations (Full Pipeline). . . . . . . 128 


\begin{abstract}
The advancement of 3D depth sensors, such as LIDAR (Light Detection And Ranging) scanners, has provided an effective alternative to traditional CAD-based and image-based approaches for 3D modeling. The output of the $3 \mathrm{D}$ depth sensors is generally 3D color point cloud and LIDAR images which is collected at the time of the LIDAR survey. However, in order to model 3D scenes under different conditions requires registering natural images with the registered LIDAR images for $2 \mathrm{D}$ image domain approaches. That is not trivial due to the repetition and ambiguity that often occur in man-made scenes as well as the variety of properties different renderings of the same subject can possess such as lighting conditions, content changes, camera sensor types, focal lengths, and exposure values. Morever, creating very high quality 3D models takes very long time to acquire and often also requires high end expensive scanners. This desertation addresses both those obstacles. For registration problem, we propose a 2D-2D matching pipeline that builds upon traditional keypoint matching techniques and uses contextual information and mid-level information to handle these challenging scenarios. For visualization problem, we proposes a novel deep learning based approach that can generate high resolution photo realistic point renderings from low resolution point clouds. The proposed method can generate high quality point rendering images very efficiently and can be used for interactive navigation of large scale 3D scenes as well as image-based localization.
\end{abstract}




\section{Chapter 1}

\section{Introduction}

Image matching is one of the fundamental problems of computer vision and image processing, it has been widely applied in many fields such as pattern recognition, 3D reconstruction and motion analysis. While matching a pair of images using corner features and RANSAC-based homography estimation generally performs well on images taken same scenes with the same conditions such as: illumination, sensor types, etc., matching multi-modality image data is a very challenging task. Image sets collected over a period of time during which the lighting conditions and scene content may have changed, different artistic renderings, varying sensor types, focal lengths, and exposure values can all contribute to visual variations in data sets. To overcome the challenging, we have developed an approach that builds on traditional methods to consider contextual information over a larger and more stable region. Registering architectural imagery is another challenging problem. Many architectural designs include repetitive elements such as rows of windows, doors, or balconies that all have similar appearances. If either local or regional descriptors are extracted for different elements of a repeating pattern, they may be the same values. This leads to ambiguity when performing descriptor comparison. We address this obstacle by taking a two-step approach, aligning pairs one dimension at a time and by abstracting images 
that originally contain many repetitive elements into a set of distinct, representative patches.

We propose a contextual framework for 2D image matching and registration using an ensemble feature. Our system is beneficial for registering image pairs that have captured the same scene but have large visual discrepancies between them. It is common to encounter challenging visual variations in image sets with artistic rendering differences or in those collected over a period of time during which the lighting conditions and scene content may have changed. Differences between images may also be caused using a variety of cameras with different sensors, focal lengths, and exposure values. Local feature matching techniques cannot always handle these difficulties, so we have developed an approach that builds on traditional methods to consider linear and histogram of gradient information over a larger, more stable region. We also present a technique for using linear features to estimate corner keypoints, or pseudo corners, that can be used for matching. Our pipeline follows this unique matching stage with homography refinement methods using edge and gradient information. Our goal is to increase the size of accurate keypoint match sets and align photographs containing a combination of man-made and natural imagery. We show that incorporating contextual information can provide complimentary information for scale invariant feature transform and boost local keypoint matching performance, as well as be used to describe corner feature points.

Matching and aligning architectural imagery is an important step for many applications but can be a difficult task due to repetitive elements often present in buildings. Many keypoint descriptor and matching methods will fail to produce distinctive descriptors for each region of man-made structures which causes ambiguity when attempting to match areas between images. In this thesis, we outline a technique for reducing the search space for matching by taking a two-step approach, aligning pairs one dimension at a time and by abstracting images that originally contain many 
repetitive elements into a set of distinct, representative patches. We also present a simple, but very effective method for computing the intra-image saliency for a single image that allows us to directly identify unique areas in an image without machine learning. We use this information to find distinctive keypoint matches across image pairs. We show that our pipeline is able to overcome many of the pitfalls encountered when using traditional keypoint and regional matching techniques on commonly encountered images of urban scenes.

Image super-resolution is also a fundamental problem in computer vision. It has a lot of applications in digital high definition television, medical imaging, satellite imaging and airborne surveillance. It is a well-known ill-posed problem due to the lack of additional information provided. In other word, it is an underdetermined inverse problem, of which solution is not unique. In this thesis, we propose a novel CNN (Convolutional Neural Network) that learn end-to-end mapping between lowresolution image and high-resolution image. We also address the problem of point based rendering via image super-resolution. This task can be considered as image enhancement.

Convolutional neural network (CNN) has been successfully applied in many areas of computer vision, including single image super-resolution (SISR). We present a new CNN-based SISR method that outperforms existing state-of-the-arts CNNbased SISR methods. We propose to apply the deeply supervised nets with incremental learning into single image super-resolution (SRDSN). The SRDSN, consisting of multiple similar residual blocks, leverages fully convolutional neural networks and deeply-supervised nets. Each residual block, containing multiple convolutional layers followed by PRELU, takes an intermediate high-resolution (HR) image and output a refined intermediate HR image which serves as the input for the next block. Finally, all the intermediate HR images are fused together to obtain a final HR image. We show that the proposed algorithm outperform state-of-the-art methods with notice- 
able visual improvements on some popular benchmark datasets.

Current state-of-the-art point rendering techniques such as splat rendering generally require very high-resolution point clouds in order to create high-quality photo realistic renderings. These can be very time consuming to acquire and oftentimes also requires high-end expensive scanners. This thesis proposes a novel deep learning based approach that can generate high resolution photo realistic point renderings from low resolution point clouds. More specifically we propose to use co-registered high quality photographs as the ground truth data to train the deep neural network for point based rendering. The proposed method can generate high quality point rendering images very efficiently and can be used for interactive navigation of large scale 3D scenes as well as image-based localization. Extensive quantitative evaluations on both synthetic and real datasets show that the proposed method outperforms state-of-the-art methods.

\subsection{Contribution}

In this dissertation, we present the following contributions to the area of image matching and image super-resolution.

1. We present a contextual matching framework for $2 \mathrm{D}$ image matching which deals with the fact that images captured by different sensors and under different conditions may actually have very different image intensity and gradient patterns surrounding corresponding points. We propose an ensemble feature that combines the contextual information with local keypoint matching methods. We match both hard and soft edge features (lines and HOGs) in these regions to increase the matching feature set size between images with visual discrepancies. Line segments represent the salient features and structures found in an image and are relatively invariant to perspective and lighting changes. However, using 
line segments creates a very minimalist representation of an image. It does not provide as much distinct information as HOG, which picks up curved structures and textures. HOG, on the other hand, is sensitive to clutter in the image so we aim to strike a balance by using both measurements. For line segment extraction, we use the Line Segment Detector method. This algorithm has been shown to accurately identify existing lines in an image without also including many false detections. This method achieves low false positive rates by incorporating line-support regions that require pixels on or near a line segment share very similar gradient orientations. Including our contextual matching method in our pipeline helps us to match stable regions across lighting and time changes in images.

2. We describe a method for matching line segments. One of the main issues encountered in line-based matching is the lack of a robust distinctive line-based feature descriptor. Lines in general have no area and the sizes of their neighborhoods are not known or are hard to determine. The length of a line segment is fragile and therefore is not a stable attribute for comparison. However, the relative orientations and locations of line segments can be computed if a proper anchor point is available. In our work, we combine SIFT and corner points with line segments to conduct line matching. By exploring multiple scales of regions and using dominant gradient information in a local neighborhood, we are able to develop a coordinate system which can be used to encode the relative locations and orientations of the line segments in local neighborhoods. We avoid including any information dependent on the unstable end points of the line segments and focus on matching groupings of segments as opposed to individual lines.

3. When the matching images contain repetitive structures that potentially lead to 
ambiguity such as urban building, we propose a method for identifying repetitive patterns and salient regions and use them for guiding the matching process. That is to say, we align rectified images one dimension at a time, reducing the search space and increasing the image matching robustness, computing intraimage saliency maps in an unsupervised manner with no training set, and directly accounting for image region saliency when quantifying keypoint match quality

4. For the single image super-resolution. We propose a novel network incorporating the concept of deeply-supervised nets for single image super-resolution. The network, consisting of multiple similar residual blocks, leverages fully convolutional neural networks and deeply-supervised nets. Each residual block, containing multiple convolutional layers followed by PRELU, takes an intermediate high-resolution (HR) image and output a refined intermediate HR image which serves as the input for the next block. Finally, all the intermediate HR images are fused together to obtain a final HR image. Extensive quantitative evaluations on popular benchmark datasets for SISR show that the proposed algorithm outperform state-of-the-art methods with noticeable visual improvements.

5. We are the first group that applies deep learning in point-based rendering. We use co-registered high quality photographs as the ground truth data to train the deep neural network for point-based rendering. We also demonstrate that our proposed method can generate high quality point rendering images very efficiently and can be used for interactive navigation of large scale 3D scenes as well as image-based localization with significantly reduced overhead 


\subsection{Organization}

This thesis is organized as follows: Chapter 2 details our method for registering 2D images with 2D images. Chapter 3 expands upon our contextual 2D- 2D matching approach to handle scenarios with highly repetitive data. Chapter 4 proposes deeplearning method for single image super-resolution. Chapter 5 presents a deep-learning based method for 3D point rendering. Chapter 6 summarizes this thesis. 


\section{Chapter 2}

\section{An Ensemble Approach to Image Matching Using Contextual Features}

\section{$2.1 \quad$ Introduction}

Matching photographs and finding image correspondences is necessary for a variety of applications in computer vision from creating structure from motion point clouds to image classification. While several methods already exist for detecting and matching sets of pixels using image intensity patterns, many will encounter difficulties when images are captured under highly varying conditions. Changes in lighting, camera exposure values, scale, scene content, and artistic styles can greatly affect local gradient information surrounding keypoints. Popular keypoint detection and matching methods such as Harris Laplacian [10] and SIFT [11] that rely on such information may not perform well under these circumstances. In difficult situations, they may provide too few matches or so many incorrect matches that a correct image alignment may not be identified after applying robust methods like Random Sample Consensus (RANSAC) [12]. Standard detector techniques may not even find a sufficient amount 


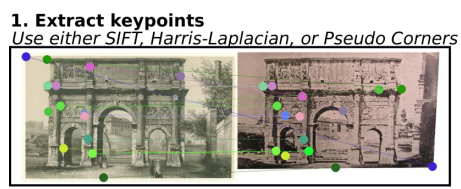

2. Identify MSER-based segments

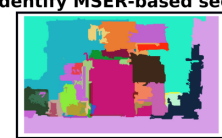

3. Find matches using contextual information
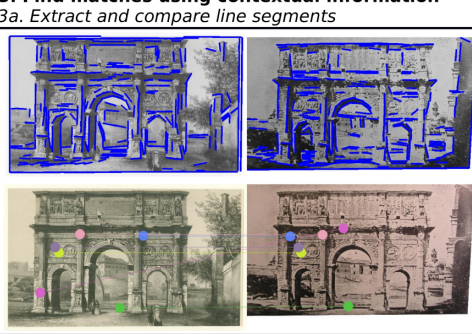

3b. Compute and compare HOG's

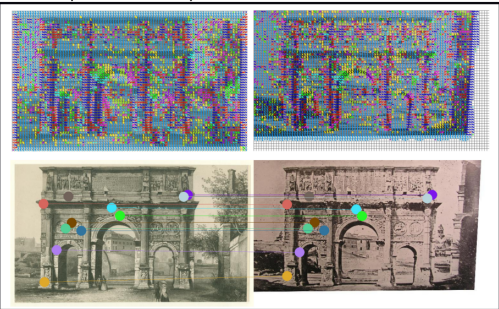

4. Align images and refine with ICP

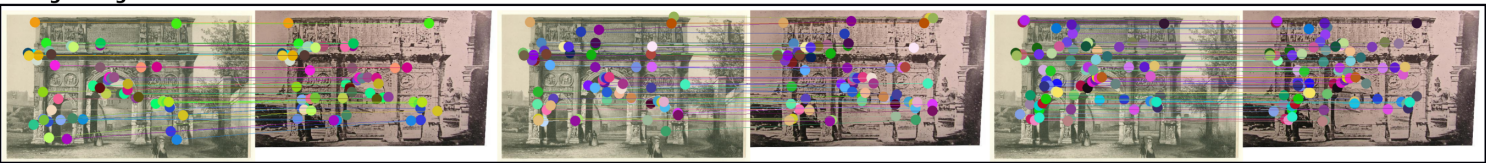

5. Global refinement aligning line segments of one image with strong gradient magnitude responses of the other
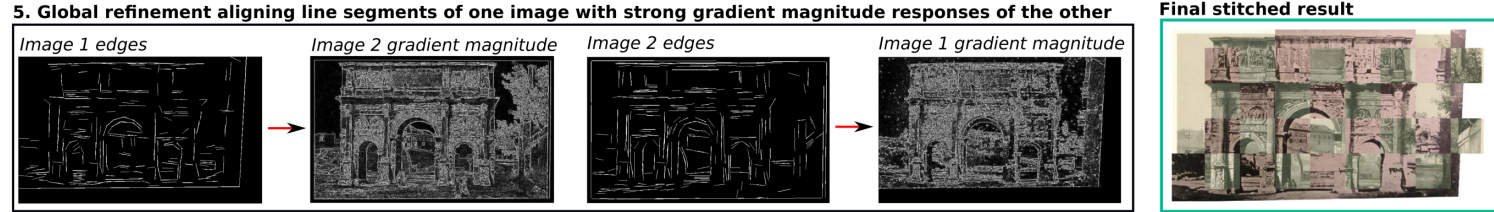

Figure 2.1: Outline of our registration pipeline. Keypoint features are first extracted. SIFT keypoints, Harris-Laplacian corners, or our proposed Pseudo-Corners can be used. If SIFT is used, keypoints are matched using the SIFT descriptor. We then search for matches using contextual features. $I_{1}$ is segmented into MSER-based neighborhoods which are used to determine neighborhood sizes for studying line segments and HOG's. (HOG information in top right image is colored according to the dominant gradient direction for each cell.) Linear and HOG information is used to verify new matches. An aligning homography is calculated and refined using ICP and gradient-based methods.

of repeatable features to match keypoints successfully. We propose a pipeline that builds upon traditional keypoint matching techniques and uses contextual information to handle these challenging scenarios. We hypothesize that using this extra regional information can help clear up a great deal of the ambiguity presented by small scale gradient information to find a larger set of matches for registration purposes. We also present a method for estimating corner keypoint locations for situations where other repeatable features may be hard to detect and use this same contextual information to match them.

All features have their own strengths and weaknesses and each may be more wellsuited for specific tasks than others [10]. On one hand, defining features on a very local scale can create a relatively large set of keypoints that are highly informative 
for matching and invariant to certain amounts of perspective and content changes. However, limiting the amount of visual information accounted for in a descriptor may obscure the differences in these local keypoint descriptors [13]. This is especially true when highly different viewing conditions, clutter, and occlusions are present, causing local visual properties to change, making it difficult to match locally-defined keypoints correctly. Using a larger scale can help define more discriminative features. By combining local and regional information into one ensemble feature, we feel we can achieve the best of both worlds by taking advantage of both precise local information and distinct regional data.

\subsubsection{Background}

Images can be aligned using information from locally defined feature descriptors, regional descriptors, direct methods that rely on global pixel-pixel correspondences, or structural features such as line segments. Locally defined keypoints use data, such as gradient direction and magnitude, from neighboring pixels within a relatively small window size. Regional descriptors use information over a larger portion of the image to describe an area and potentially match it to corresponding regions in other images. Global matching techniques, such as optical flow and Fourier-based alignment, can provide a very accurate image alignment, but generally require a good initialization to be successful [3].

We try to take advantage of the strengths of several of these methods by combining them together into one ensemble feature matching and alignment refinement approach that uses local, regional, global, and structural information. 


\section{Local Matching Methods}

The Harris detector and use of the Hessian matrix are two classical methods for finding local interest points in images [3] [14] [10]. Both detectors use the second moment matrix to study the gradients of pixels surrounding an interest point. The scales for points' feature descriptors are often chosen using the Laplacian [15]. These methods work to identify local areas with significant visual changes, such as corners, and are fairly invariant to lighting changes. However, these early methods were shown to be sensitive to large scale changes and had limited affine invariance, which led to the development of more sophisticated detectors and descriptors. For example, the Scale Invariant Feature Transforms method (SIFT) [11] is a commonly used and robust local feature matching technique that assigns a scale and orientation to each interest point. A descriptor is constructed based on local image gradients that is generally unique to a single area in the image. The neighborhood size used to construct the descriptor is usually around $16 \times 16$ pixels at the appropriate image scale. Several groups have expanded on the SIFT descriptor to develop feature matching methods that are faster and more robust to various transformations [16] [17] [18].

The relatively small window sizes used by these methods to create keypoint descriptors can be beneficial in many cases. They will be able to identify locally distinct areas accurately. However, cases where image pairs have large scale, lighting, and/or content changes may require a larger window size to correctly pinpoint very distinct keypoint matches since the images will have so many visual differences.

\section{Regional Matching Methods}

Several methods for matching and aligning photographs have expanded on these locally defined feature descriptors to use visual information covering a larger region within images. Yang et al. [7] designed a pipeline using bootstrapping and region growing to search for a dense set of corner and edge features. They use these fea- 

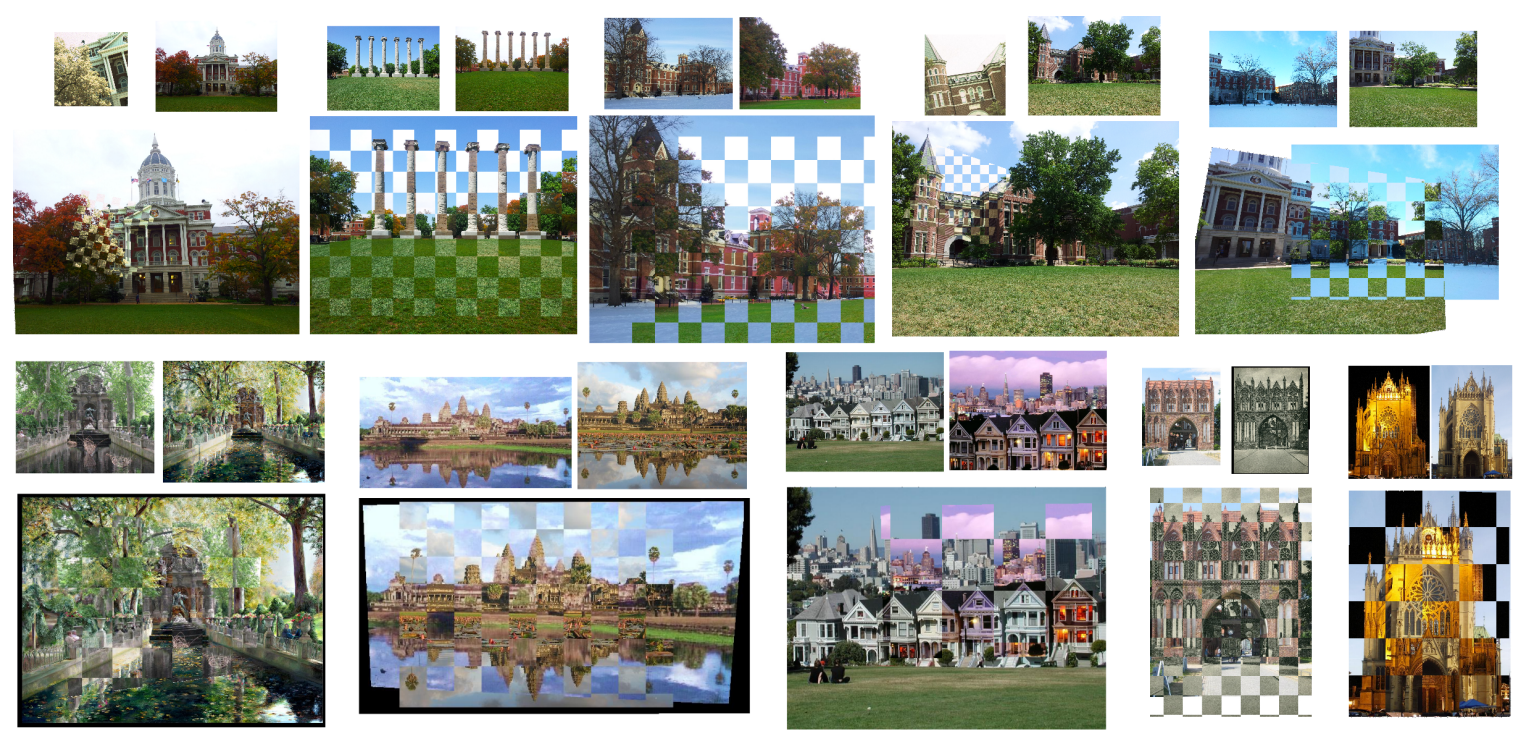

Figure 2.2: Registration results after applying our pipeline to several challenging image pairs from our dataset as well as from the datasets provided in [1] and [2]. Original images are shown above registered images.

tures to compute a global homography relating two images. Since this method uses a single homography to overlay an image pair, it is best-suited for planar-approximate scenes. Hacohen et at. [19] also identifies dense matches across image pairs. They align image patches by searching across a set of transformations such as rotations and translations that overlay pairs of patches as well as possible and use groupings of matches to calculate the coherence of a region.

Other groups have developed region detectors and descriptors that rely on lessstructured features. MSER's are used to identify image neighborhoods that are visually similar and contrast greatly with adjacent regions. The SIFT descriptor can be used to describe and match corresponding MSER's in image pairs [20]. MSER is affine invariant but has been shown to be biased to "round" shapes [21]. If an image has repetitive patterns, such as a row of windows, it is possible that the MSER feature will be ambiguous if its size is not large enough. Shape contexts [22] describe feature points that are uniformly sampled along contours based on nearby keypoint's relative locations. These groupings of points are used to find optimal image corre- 
spondences and are useful for object recognition. Contours throughout the image must be correctly extracted, which is not always an easy task.

Measuring the similarity of all overlapping pixels is another approach for region matching. Normalized cross correlation, the sum of squared differences, and the sum of absolute differences all perform a pixel-by-pixel comparison and can be used to find the best alignment for an image pair [23]. Histograms of gradients (HOG) have also been used as unstructured region descriptors for image alignment verification and object recognition [24]. While these methods can be very informative, scale and orientation information should be known in order to use them efficiently. To maximize their robustness, and to avoid exhaustively searching for overlapping regions, they can be combined with other information or descriptors to provide this information.

Another class of mid-level, regional matching approaches is to identify symmetrical elements and self-similarities in images. This is especially useful when working with architectural data that often times contains repetitive structures [25]. Hauagge and Snavely search for horizontal, vertical, and rotational symmetries about various axises and scales across images [2]. Self-similarities can even be identified in patterns of colors, edges, and repeated visual elements in non-architectural imagery by measuring how similar a small region is to parts of the larger surrounding region [26].

Machine learning techniques can also be used to identify unique regions in photographs, paintings, and 3D models to match imagery with highly varying properties and to perform object recognition $[1,27,28]$. These mid-level discriminative features ideally occur often enough in the data to be learned, but are different enough from the rest of the imagery to be clearly located in photographs and models with visual dissimilarities. Alignment estimates can even be made between multi-modal data such as paintings and photo-realistic 3D models by using the gist scene descriptor and edge information of different regions [29]. 


\section{Linear Features}

Extracting long linear features is another approach used for matching images. This can be a very difficult task as line features are relatively fragile and their lengths may vary with changing viewpoints and lighting conditions. Wang et al. [30] match clusters of line segments, or line signatures, in image pairs using the assumption that local neighborhoods of strong structural features will maintain the same relative arrangements throughout baseline and lighting changes. Fan et al. [31] also match lines by using spatially proximate features. They consider two lines to be a matching pair if they have similar distance ratios to neighboring keypoints that are known matches. Since each potential linear match requires nearby correctly matched keypoints, this method is dependent on having a relatively large number of correct feature point correspondences. Color histograms of the color profiles surrounding a line segment have also been used for line matching under the observation that the changes in color between two intersecting planes is relatively invariant to camera motion [32].

\subsubsection{Overview and Contributions}

In this paper we present a framework for using contextual information for image matching with the goal of increasing the number of keypoint matches found between challenging image pairs in order to find an aligning transformation relating the images. We mainly work with piece-wise planar man-made scenes that need not be time adjacent, examples of which are shown in Figure 2.2. For planar-approximate image patches, we believe that using relatively large regions for keypoint comparison helps features remain more distinct throughout such condition changes. Our contextual framework for registering images can take three forms. The first is to build upon and complement existing sparse local feature matching techniques such as SIFT. We describe the surrounding regions of keypoints in terms of salient structural features 
via line segment extraction and their rich texture information using histograms of gradients (HOG). These neighborhood sizes are determined adaptively using maximally stable extremal regions (MSER) [33]. To eliminate the requirement that we incorporate an existing matching algorithm, we can also use our regional contextual information to describe and match Harris-Laplacian corners. The third option is to estimate corner locations using extracted line segments, which we call Pseudo Corners, freeing this algorithm from any dependencies on existing detectors or descriptors. After combining several local keypoint and regional template matching techniques to make an educated estimate about the image alignment, we follow up with iterative and global refinement stages using corner, edge, and gradient information across the entire image planes. Our pipeline is outlined in Figure 2.1.

The contributions of our registration method lie in the combination of methods used including the structure of our contextual matching framework and the way we incorporate this framework with local keypoint matching to create an ensemble feature. We propose using robust SIFT feature points or plentiful corner keypoints as anchor points for exploring potentially matching regions by comparing both HOG's and line segments. The MSER's that encompass each SIFT or corner keypoint, and that are often used to identify homogeneous regions, give us a general idea of the dimensions of local planar patches and what the neighborhood size should be for stable matching. We match both hard and soft edge features (lines and HOG's) in these regions to increase the matching feature set size between images with visual discrepancies. Line segments represent the salient features and structures found in an image and are relatively invariant to perspective and lighting changes. However, using line segments creates a very minimalist representation of an image. It does not provide as much distinct information as HOG, which picks up curved structures and textures. HOG, on the other hand, is sensitive to clutter in the image. For line segment extraction, we use the Line Segment Detector method [34]. This algorithm 
has been shown to accurately identify existing lines in an image without also including many false detections due to its incorporation of line-support regions that require pixels on or near a line segment share very similar gradient orientations. Including this method in our work helps us to match stable regions across lighting and time changes in the images.

One of the main issues encountered in line-based matching is the lack of a robust distinctive line-based feature descriptor. Lines in general have no area and the sizes of their neighborhoods are not known or are hard to determine. The length of a line segment is fragile and therefore is not a stable attribute for comparison. However, the relative orientations and locations of line segments can be computed if a proper anchor point is available. In this paper, we combine SIFT and corner points with line segments to conduct line matching. By exploring multiple scales of regions and using dominant gradient information in a local neighborhood, we are able to develop a coordinate system which can be used to encode the relative locations and orientations of the line segments in local neighborhoods. We avoid including any information dependent on the unstable end points of the line segments and focus on matching groupings of segments as opposed to individual lines. Our experimental results show that making use of line segments in this fashion can provide accurate information for matching on a large variety of imagery.

By using a variety of methods to find image pair correspondences and making no assumptions about a scene's structure, we have developed a robust pipeline that is capable of registering images with varying subjects including highly regularized architecture with repeating patterns and more natural, unorganized subjects combining buildings with curved features and non-standard designs and added foliage. 


\subsubsection{Notation}

For simplicity during our discussion, we will denote an image pair as $I_{1}$ and $I_{2}$. A feature point in $I_{1}$ is represented as $A$ and its two closest matches in $I_{2}$ are $B$ and $C$. When $I_{2}$ is transformed to $I_{1}$ 's coordinate system using a homography, $H$, we will represent it as $I_{2}^{\prime}$.

Throughout our discussion, we use the term "contextual" to refer to the set of matches verified using both linear and HOG contextual information. "Ensemble" will refer to the set of features combining both SIFT matches and contextually-verified matches.

\subsection{Contextual Feature Matching}

The goal of our method is to extract highly distinct interest points and obtain a large number of correspondences that can be used to correctly register image pairs [13]. Our pipeline begins by using regional contextual information to match keypoints. We explore using three different types of keypoints as anchors for matching contextual descriptors. The first approach we study is matching existing locally defined keypoint descriptors, such as SIFT, and expanding upon them with our contextual information to create an ensemble feature. The second keypoint we test is Harris-Laplacian using only our contextual work for matching. The third, Pseudo Corners, is a new keypoint type we propose which is identified using line segments. We then identify inliers in the match set from any of these methods using geometric verification, globally estimate and refine the image alignment, and increase our set of matches. 

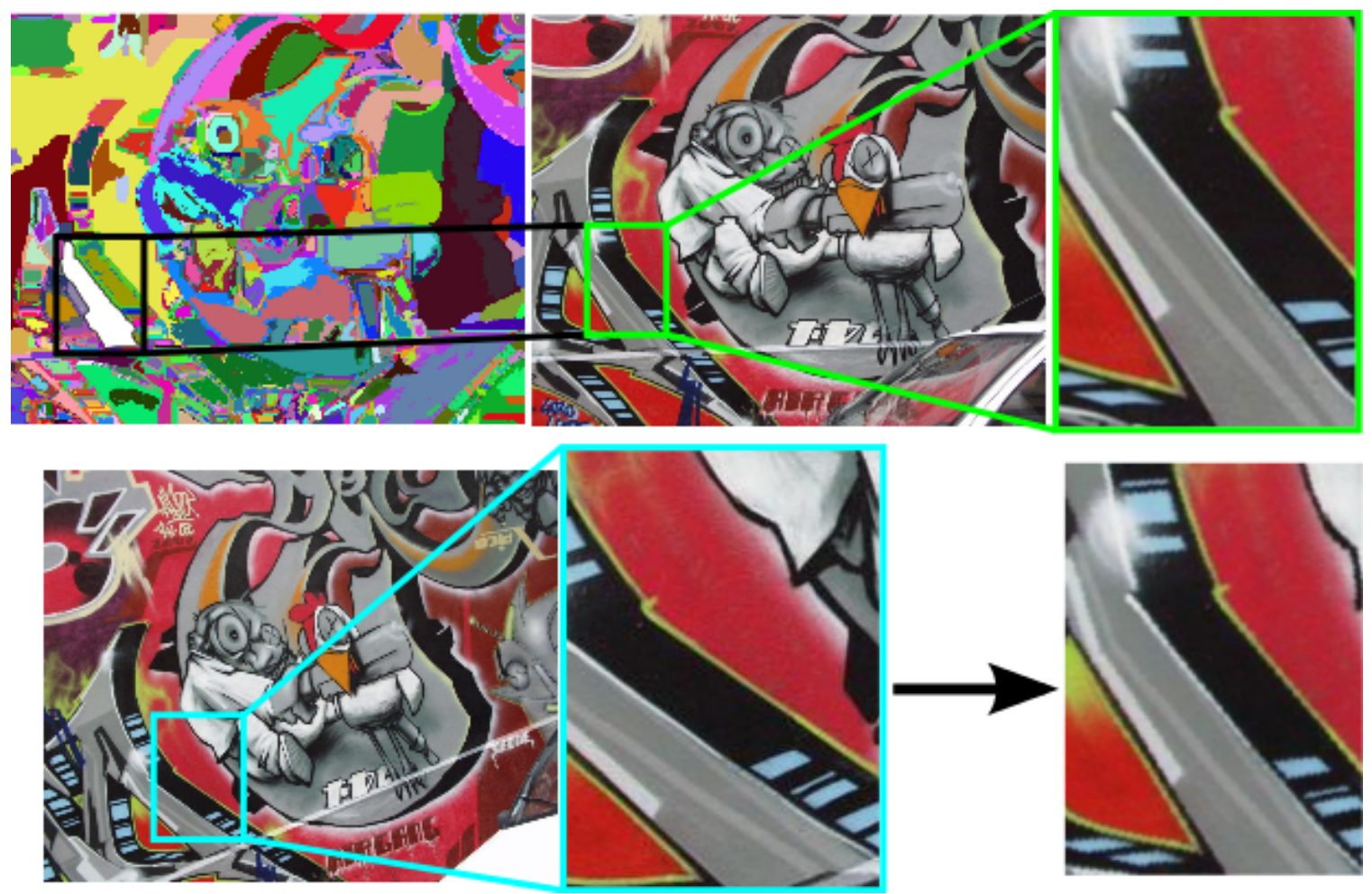

Figure 2.3: Mapping bounding box of MSER neighborhood in $I_{1}$ to $I_{2}$. Top: MSER segments and MSER defined neighborhood in $I_{1}$. MSER segment being used is shown in white. Bottom: Original MSER neighborhood in $I_{2}$ and $I_{2}$ 's patch after it has been transformed to the same coordinate system as $I_{1}$ 's patch via a scaling and rotation. The patch in $I_{2}$ is extracted at multiple scales and each patch is rotated such that its $\mathrm{x}$-axis points along the dominant gradient direction in the patch.

\subsubsection{Adaptive MSER Neighborhoods}

We use MSER's to determine the neighborhood that is considered during contextual matching. MSER's are often used to find homogeneous areas in images that contain distinctive information that is useful for matching $[35,36]$. An ideal neighborhood needs to contain enough regional information to clear up any ambiguity at the local keypoint level but not include so much information that no regions actually match. We also want every pixel in an image to belong to an MSER so that we have a "stable" neighborhood ready to select for every potential keypoint match. To find potential MSER's that satisfy these criteria, we create a Gaussian pyramid for an image and extract MSER's at each level, as shown in Figure 2.4. At the original 

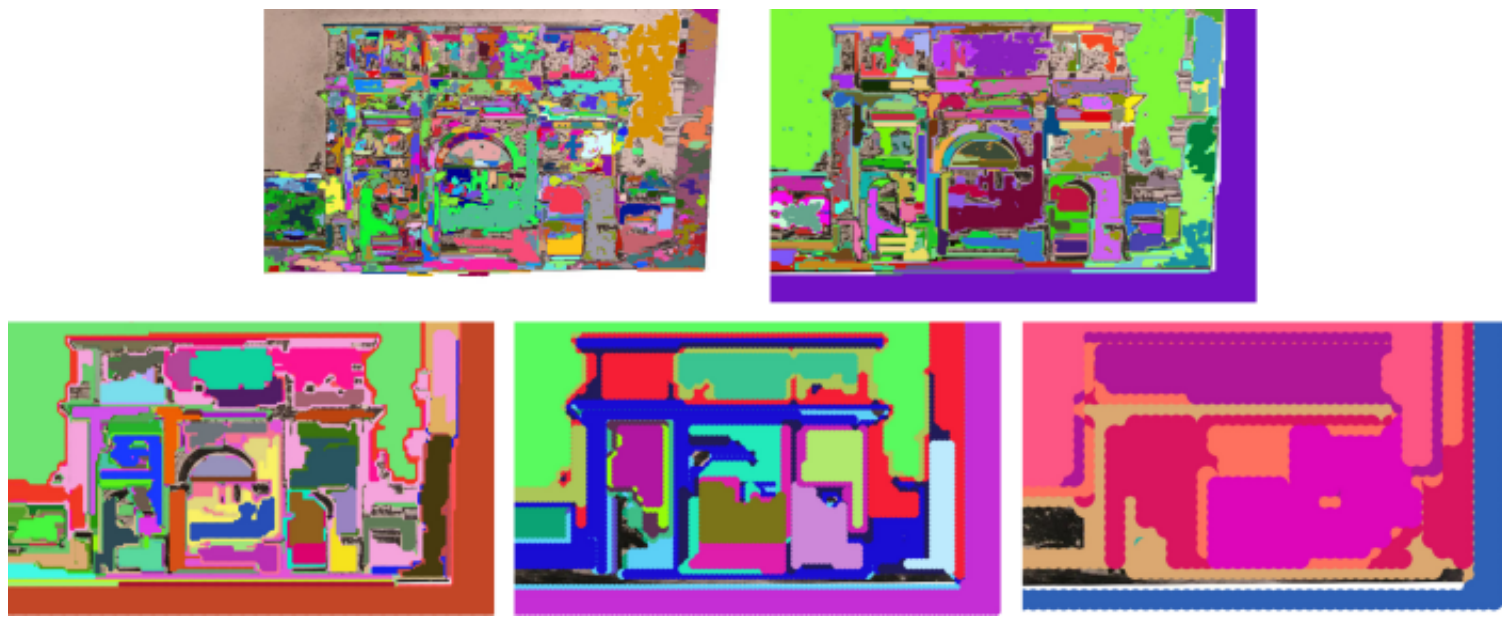

Figure 2.4: MSER's extracted at different levels of a Gaussian pyramid. Segments are combined from different levels to create adaptive MSER segments shown in Figure 2.1.

image resolution, we get many MSER's that tend to be relatively small. Also, initially, not all pixels are assigned to an MSER. As we move down the pyramid and extract MSER's on increasingly blurred images, we end up with fewer, larger regions and more pixels assigned to a region. Just to ensure that every pixel is indeed assigned to a region, we have one post-processing step after MSER's are extracted at each level. Connected components of pixels that have no region label are identified. Each component is merged with the region that touches the highest number of pixels along the component's perimeter. In general, segmentation is a very difficult task, but this adaptive MSER labeling gives us a reasonable approximation of the regions we need.

To help achieve our goal of assigning every pixel to a mid-sized MSER, we allow different pixels in the image to be assigned to MSER's from different Gaussian pyramid levels. Initially, every pixel is assigned its corresponding label at the bottom level (original image resolution) of the pyramid. Then we begin moving up the pyramid. If a pixel's corresponding region in the next level of the pyramid has an area that is no more than $10 \%$ of the image area (a threshold determined experimentally for our work), the pixel takes on the higher level region area.

The keypoint's neighborhood in $I_{1}$ used during contextual matching is an orthog- 
onal bounding box that encompasses an entire chosen MSER. Using this bounding box essentially grows the region slightly and allows us to take into account boundary information surrounding the distinctive area. During the matching stage, the MSER bounding box in $I_{1}$ is mapped to $I_{2}$ at multiple relative scales to take into account scale changes between the images. The bounding boxes are oriented such that each one's x-axis is aligned along the dominant gradient direction within the image patch. An example of using this information to find a corresponding region between $I_{1}$ and $I_{2}$ is shown in Figure 2.3.

\subsubsection{Contextual Line Segments}

We use linear contextual matching to describe potential matches by searching for and matching line segments near keypoints. Stable line segments in the MSER-based neighborhood of potentially matching keypoints are identified using the Line Segment Detector method [34]. Line segments are extracted from $I_{1}$ and $I_{2}$ at three different levels of a Gaussian pyramid. The lines found at each Gaussian level are all combined into one set of lines. Extracting lines from multiple image scales helps to address the fragile nature of line segments and gives us more information for matching. Each line segment must have a length of at least $0.03 * \max \left(\right.$ image $_{\text {width }}$, image $\left._{\text {height }}\right)$ and its midpoint must be located within the selected MSER bounding box to be taken into account. All the stable line segments in this neighborhood are transformed to a polar coordinate system determined by the neighborhood's dominant gradient orientation and normalized by the neighborhood's dimensions and are represented as $(\rho, \theta)$. We then match groupings of line segments in corresponding neighborhoods using Hungarian graph matching [37]. Figure 2.5 outlines this process. Two line segments $\left(l_{A}, l_{B}\right)$ are considered to be similar if they have a low dissimilarity score 
according to Equation 2.1.

$$
\operatorname{dissim}\left(l_{A}, l_{B}\right)=\left(\rho_{A}-\rho_{B}\right)^{2}+\omega\left(\theta_{A}-\theta_{B}\right)^{2}
$$

$\omega$ is a weight parameter that normalizes the range of $\theta(\theta \in[0,2 p i])$ to that of $\rho$ which is based on the contextual neighborhood dimensions. This dissimilarity measure is only calculated if at least three lines are identified in the contextual neighborhood of the keypoint. If a required percentage of the line segments have low dissimilarity scores, the keypoint match is saved. Table I shows how the precision of linear contextually verified matches changes as the required percentage of matching lines in a region and the dissimilarity score thresholds change. These values were obtained by running our method on the symmetry dataset in [2]. Figure 2.6 shows a set of matching lines.

Table 2.1: Average Precision on Symmetry Dataset for Varying Graph Matching Parameters - dissimilarity score and threshold on required \% of matching lines

\begin{tabular}{c|c|c|c}
- & dissim $=1.0$ & dissim $=2.0$ & dissim $=3.0$ \\
\hline $10 \%$ & 0.552 & 0.617 & 0.412 \\
$30 \%$ & 0.371 & 0.426 & 0.365 \\
$60 \%$ & 0.000 & 0.081 & 0.081
\end{tabular}

\subsubsection{Contextual HOG}

Our second approach for contextual matching takes advantage of every pixel in MSERbased neighborhoods surrounding potential keypoint matches. The potentially matching neighborhood in the second image is calculated at multiple scales and its orientation is determined using the region's dominant gradient direction, giving us a region that contains the same content and is based in the same coordinate system.

To measure how well the two neighborhoods match, we begin by computing histograms of gradients for each image patch, Just as was discussed in Section 2.2.2, a 
Line Segments

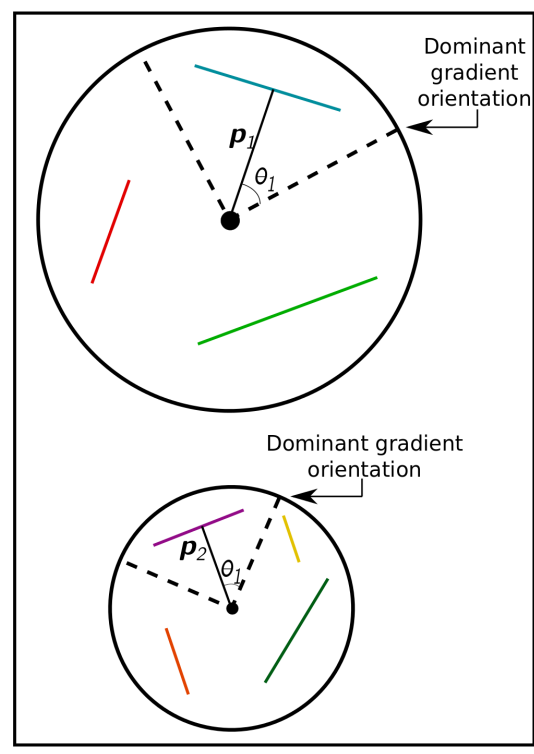

Polar Coordinates

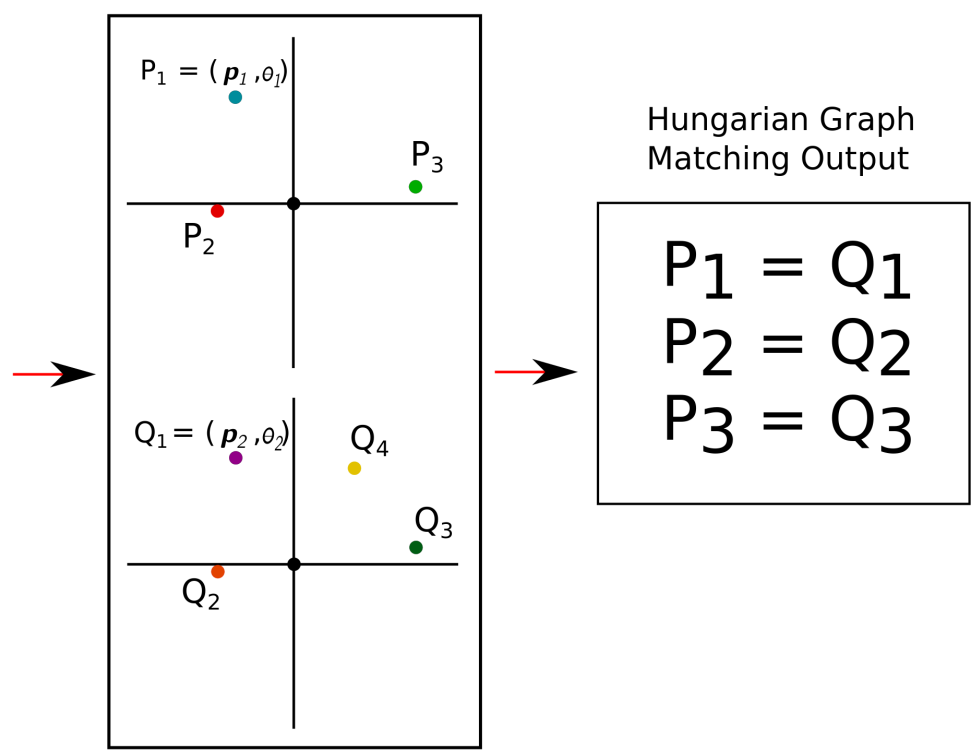

Figure 2.5: Matching lines in the neighborhood of a feature point. Left: Line segments are identified within neighborhoods centering a potential match. The keypoint is shown in black in both neighborhoods and the relative scales are represented by black circles. Middle: Line segments are converted to polar coordinates based on their distance from the keypoint and their orientation in relation to neighborhood's dominant gradient direction. Right: Hungarian graph matching is applied to the set of polar coordinates to figure out the optimal set of matching lines.

three-level Gaussian pyramid is built for the corresponding neighborhoods. A vector is constructed for each HOG cell by concatenating its nine bin values and normalizing using the vector's magnitude. We calculate the L2 distance between the vectors for corresponding cells in the matching neighborhoods across all levels of the pyramid. If the average of all these distances is under a defined threshold (threshold $=0.5$ for our experiments) we say that the neighborhoods surrounding the match have a strong correspondence and save the keypoint match. By requiring a region to have matching HOG's along multiple scales, we are placing a stricter requirement on the matching criteria thereby removing noise that may be present when only one scale is used. Figure 2.6 shows matching HOG cells for two corresponding neighborhoods.

This method makes no assumption about the structure of a region. It can be used to find features in both natural, highly varied regions such as areas of images 

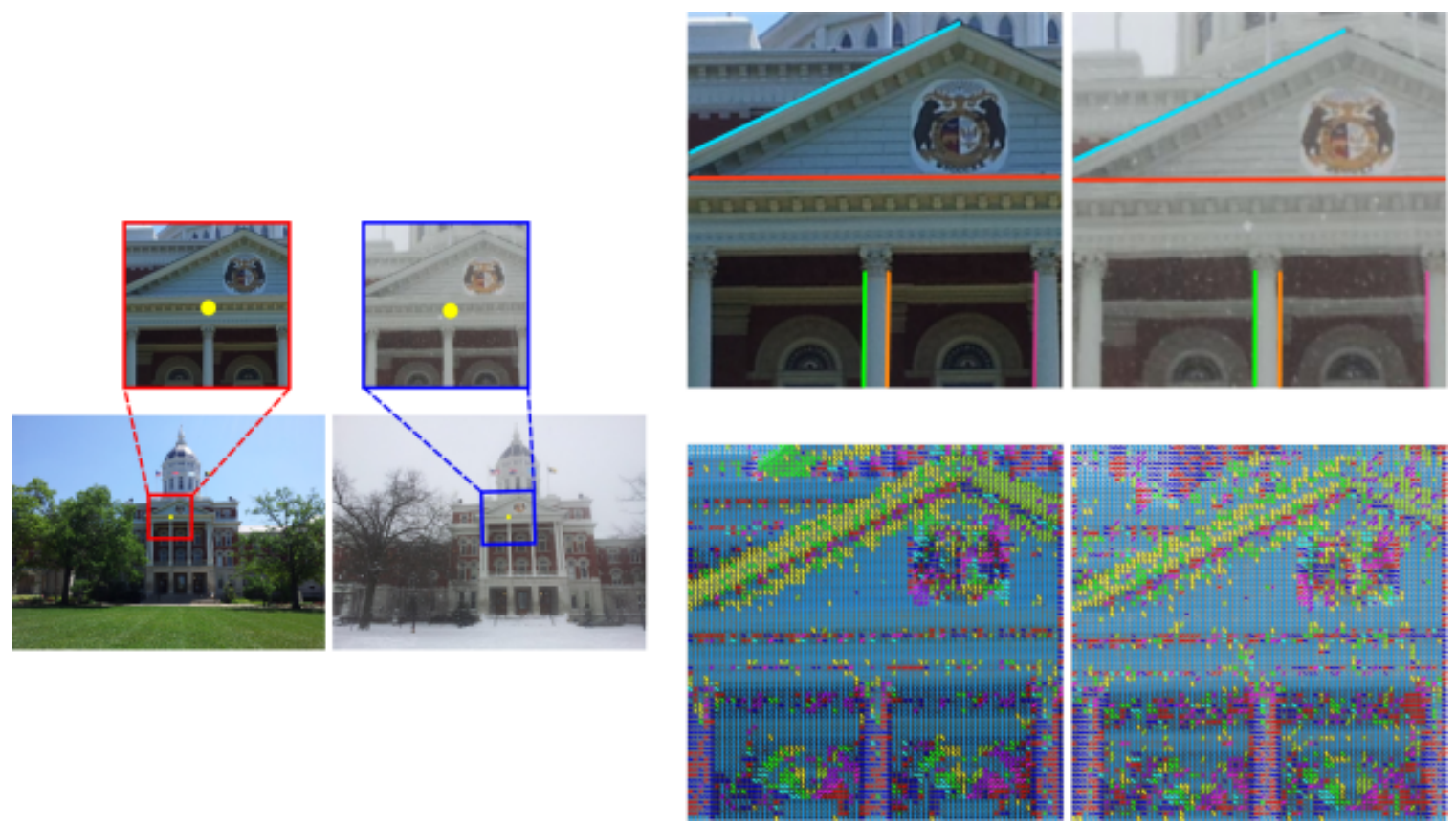

Figure 2.6: Identifying matching neighborhoods for contextual matching. This example uses a SIFT match as an anchor for exploring contextual neighborhoods. Left: Two images are matched and share a corresponding point, shown in yellow. This keypoint pair has a distinctiveness ratio between 0.7 and 0.9 and is not verified by SIFT. Top Right: Matching line segments within neighborhood. Bottom Right: HOG descriptors for cells in the neighborhood. Cells are colored according to dominant gradient direction. The line orientation in each cell shows the cell's dominant gradient direction.

containing trees as well as in areas containing uniform architecture.

\subsubsection{Ensemble SIFT}

The first way we incorporate contextual information in our pipeline begins with obtaining an initial set of matches for an image pair using SIFT, a very robust and scale invariant blob detector that describes the rich and varied gradient information surrounding an interest point. In the traditional SIFT pipeline, $A$ and $B$ are identified as a true match if the distance between their descriptors passes a distinctiveness ratio

test (i.e. $\left.\frac{\left\|A_{d e s}-B_{d e s}\right\|}{\left\|A_{d e s}-C_{d e s}\right\|}<0.7\right)$. When matching photographs with highly varying image properties, we may not obtain enough keypoint matches, or enough correct matches, 
that are very distinct to unearth a transformation mapping the images to each other. In order to identify correct point matches that may not pass the ratio test (and increase our pool of matches), we also take into account contextual information. This entails measuring the similarities of the larger neighborhoods surrounding a potential match. Sample matching neighborhoods are shown in Figure 2.6. We can use our two different types of contextual descriptors to describe these larger neighborhoods, taking advantage of hard linear features and soft histograms of gradients (HOG). Our SIFT + line segment method is robust to lighting and content changes and helps by identifying salient linear features. Our SIFT + HOG method is useful for matching regions that lack dominant lines but are rich in texture. These methods are both used to clear up some of the ambiguity between possible keypoint matches. If the neighborhoods of the matches with relatively higher distinctiveness ratios $(0.7<$ ratio $<0.9)$ match well using one of our measures, we include them in our list of putative correspondences that are used for future registration. Figure 2.7 outlines how we use the ratio test for collecting matches. An example of how using our contextual features in addition to SIFT can help improve alignment is shown in Figure 2.8.

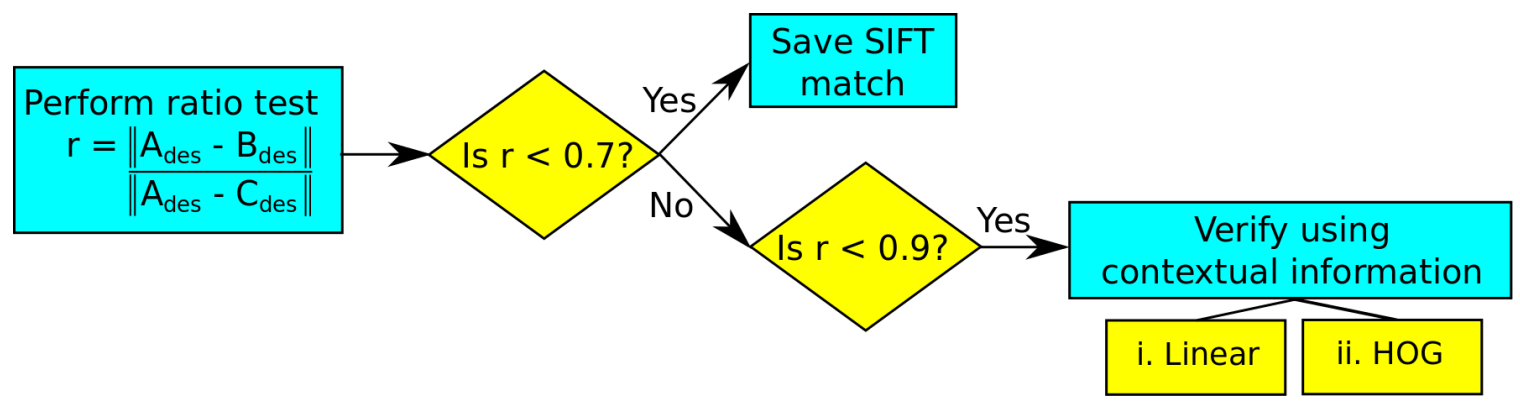

Figure 2.7: Outline of how we use the distinctiveness ratio test to collect matches and to determine when to use contextual matching in conjunction with the SIFT matching scheme. 

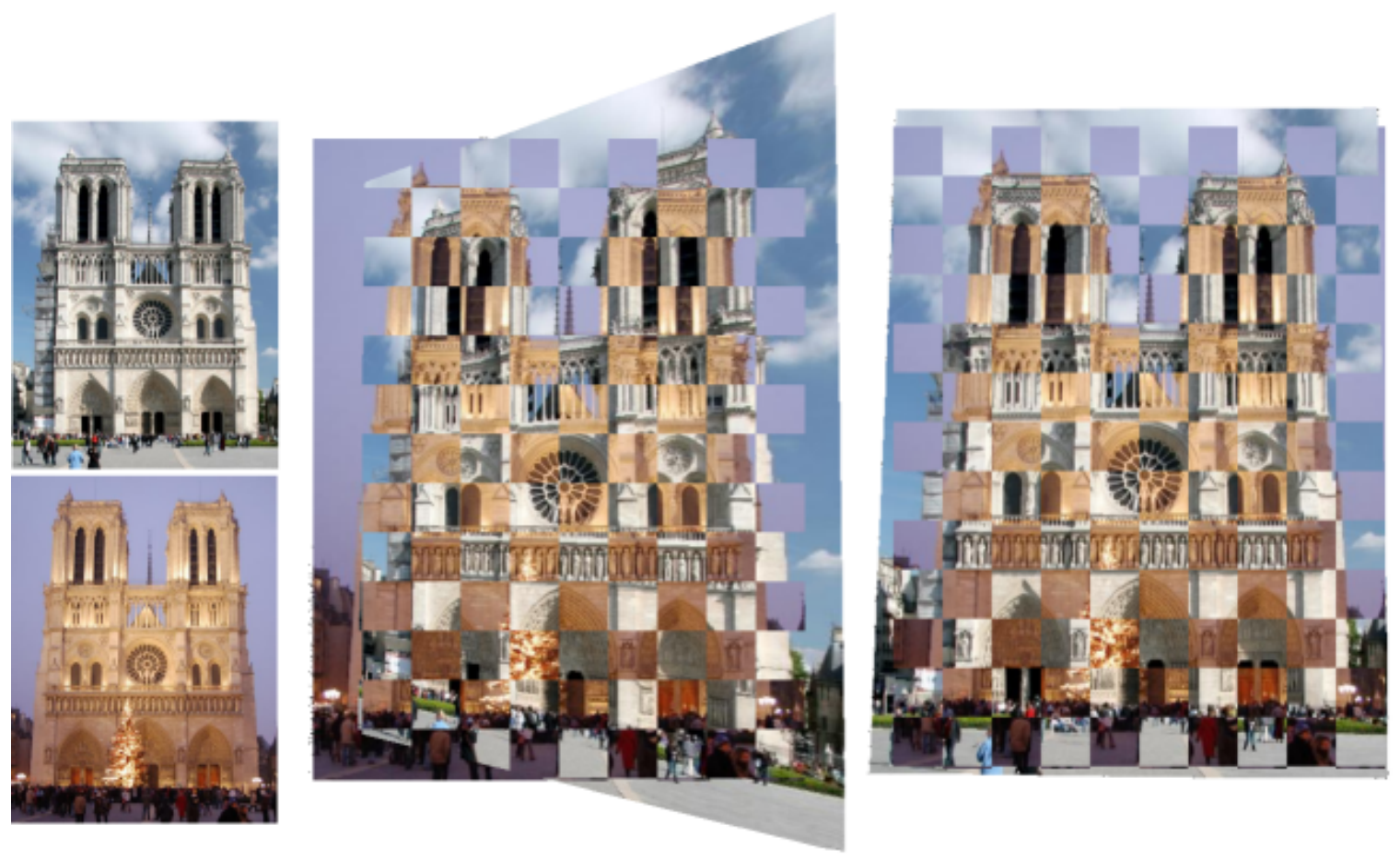

Figure 2.8: Image alignment improvement using our ensemble SIFT method. Left: Image pair to be aligned. Center: Image alignment using only SIFT features. Right: Image alignment using our ensemble features.

\subsubsection{Contextual Harris-Laplacian}

To eliminate our dependency on SIFT so that we can handle cases where an insufficient number of potential SIFT matches exists, we can also apply our contextual matching scheme to corner matching. In this scenario, we extract Harris-Laplacian corners from a pair of images and the Line Segment and HOG neighborhoods surrounding each detected keypoint. For every Harris-Laplacian corner in $I_{1}$, we compare its Line Segment neighborhood and its HOG neighborhood to the respective contextual descriptors of every corner in $I_{2}$. Neighborhoods are extracted at multiple scales as discussed in Section 2.2.2. Pairs of corners with a sufficiently low Euclidean distance between either their Line Segment or HOG-based descriptors are saved as matches. 


\subsubsection{Identifying Pseudo Corners}

We may also encounter cases where the scale, lighting, content, or rendering style changes between an image pair are so great that it is difficult to extract repeatable SIFT keypoints or Harris-Laplacian corners in both images, leaving us with insufficient keypoint information to work with for matching and registration. For these scenarios, we propose using line segments extracted from the images to estimate corner locations, which we refer to as Pseudo Corner keypoints. Line segments are extracted at multiple scales and merged together. Two segments are connected into one larger line segment if they are near collinear and are in close proximity. Similarly, two line segments will be merged into a single line segment if they are near parallel and are in close proximity. We can also incorporate a saliency requirement on the line segments used by thresholding out lines with average gradient magnitude values below a certain value as done in [31]. Our new corner keypoints are the intersection points between nearby segments. We avoid using the inaccurate intersection points of near parallel segments by restricting that any Pseudo Corner must be within a certain distance of each of the line segments used to find it. This process is especially tuned to planar, architectural scenes where we can have a high degree of confidence that the intersection point of segments laying on the same plane will correspond to a real corner. We match our Pseudo Corners using the same approach described in Section 2.2.5 by comparing the contextual neighborhoods of keypoints in the image pair. An example of our results using this approach to align a difficult image pair entitled Vatican is shown in Figure 2.9 Bottom. We can see from Figure 2.15 that this image pair is not registered well using the SIFT-based ensemble method or the contextual Harris-Laplacian method that are discussed earlier in this paper. Figure 2.9 Top shows the matches identified using our ensemble SIFT method. The middle rows show the line segments extracted from the images and the Pseudo Corners identified. The bottom image shows the image pair aligned using matched Pseudo Corners to 
estimate the pair's homography.

\subsection{Homography Estimation, Refinement, and Ver- ification}

All 2D matches identified using any form of our ensemble approach are used together to calculate a homography relating the image pair. The initial homography matrix is computed using RANSAC and Direct Linear Transform (DLT) [38]. All 2D matches found so far are geometrically verified by calculating the homography relating the image pair and identifying inlying matches. Multiple homographies are calculated for each image pair to take into account various 3D planes that may be represented in the images [39]. Each homography matrix $H_{i}$ is computed using RANSAC and the chosen solution is the transformation matrix supported by the largest consensus set among the 2D matches. Matches that support a particular homography are removed and are not considered in future homography calculations.

Our iterative approach tries to find a balance between single, global homography calculations that are limited to planar-approximate scenes and multiple-homography methods that explicitly require a planar segmentation of photographs $[40,41]$ or more complicated image deformation models [42-46]. Generally, our iterative approach chooses matches belonging to different planes on different iterations as shown in Figure 2.10, but we do not complicate our method by enforcing such constraints. We conduct iterative homography fitting for the remaining features (i.e. features that are considered outliers based on the homographies estimated in the previous loops).

We take a two step approach to refine our image alignment that deviates from the popular approach of reestimating each $H_{i}$ based on the reprojection error of the match set. We begin by searching for a dense set of matches on a relatively local 
scale. This first stage gives sets of keypoints the flexibility to search for correct matches along a variety of directions and distances. This is followed up by computing an optimal global alignment that is more rigid, but represents a general consensus for $H_{i}$ throughout the image planes.

For refinement with local information, we apply the iterative closest point (ICP) algorithm [47] on the edge and corner pixels of $I_{1}$ and find their best matches in $I_{2}$. Edge points are identified by extracting line segments and uniformly sampling them. Ideally, by finding edge points using the Line Segment Detector algorithm, we are working with "strong" edges. Edges and corners in $I_{1}$ are transformed to the matching image plane using $H_{i}$ and are compared to all of $I_{2}$ 's keypoints within a specified search radius. Pairs of keypoints with SIFT descriptor distances passing the traditional distinctiveness ratio test are saved. $H_{i}$ is re-estimated using RANSAC and DLT. The search radius for matching keypoints plays an important role in our optimization algorithm. If the current homography estimation is far away from the optimum, we would like to have a large initial radius. On the other hand, if the current estimation is close to the optimum, a smaller radius is all that is necessary. However, this information is not known a priori, so we start a relatively large radius $\left(10 \%\right.$ of $\max \left(\right.$ image $_{\text {width }}$, image $\left.\left._{\text {height }}\right)\right)$ and divide it in half for each iteration of our refinement.

After reestimating $H_{i}$, we check the new alignment by comparing HOG's across the entire overlapping region of the two images within the bounds set by the matches used to compute $H_{i}$. If the relative HOG distance after performing ICP is higher than that of the alignment before running ICP, the new alignment is rejected. This is essentially a quality control step to ensure that the refinement stage does not lead to an alignment that diverges from the best solution we can find.

We next use gradient information from the image pair to perform one last global alignment. The gradient magnitude field for $I_{1}$ and the binary edge image for $I_{2}$ 
are computed. We wish to find the homography matrix that aligns the edge points of each image with the strongest gradient responses in the matching image as much as possible. The varying strengths of $I_{1}$ 's gradient field are used to guide $I_{2}$ 's edges to their ideal alignment and vice versa. To accomplish this, $H_{i}$ is perturbed using Levenberg-Marquardt Optimization to maximize Equation 2.2. Only edge points that are within the bounded region of the image that $H_{i}$ represents are considered in Equation 2.2.

$$
\begin{aligned}
E=\underset{H_{i}}{\arg \max } & \left(\sum_{j=0}^{\# I_{2} \text { edges }}\left\|\nabla I_{1}\left(H_{i}^{\prime} p t_{j}\right)\right\|\right. \\
& \left.+\sum_{k=0}^{\# I_{1} \text { edges }}\left\|\nabla I_{2}\left(H_{i} p t_{k}\right)\right\|\right)
\end{aligned}
$$

Figure 2.11 displays gradient information that can guide our alignment and the result of using this approach. This second stage of refinement is applied at the end of our pipeline after we have already established a relatively strong estimate of the image pair alignment. Line segments in one image need to be transformed closely to a linearly shaped area of relatively strong gradient responses in the matching image for these pixels to contribute to the refining of the image alignment. Equation 2.2 tries to find a general agreement amongst the transformation of all the line segments in the overlapping regions of the images. The combination of using the Line Segment Detector method, which is robust to noise, and searching for a general consensus amongst all the line segments in the images helps our optimization avoid being overly influenced by noise and clutter in the images. Figures 2.1, 2.9, and 2.11 show examples of lines extracted using the Line Segment Detector method. We can see in these cases that the lines picked up mainly correspond to structures that exist in both images despite very different qualities in the photograph pairs. We pick up very few lines along the ground, trees, people, or other clutter or noise in the images. 
The benefit of using these types of refinement techniques instead of trying to simply minimize a match set's reprojection error is apparent when the initial point correspondences are very sparse or confined to one section of the image pair. In this situation, we are very limited in how much we can actually refine our transformation. On the other hand, our refinement approach that considers edges, corners, lines, and gradient information takes into account visual properties spanning the majority of the image planes regardless of the distribution of our point correspondences.

We have several different criteria for verifying each $H_{i}$. We first make sure that the points used to calculate the homography matrix are not collinear. Once the matrix is computed, we also take into account the layout of the transformed images and the numerical properties of the homography matrix. To check against our layout criteria, we project the four corners of $I_{2}$ to $I_{1}$ 's image plane using $H_{i}$. The top left and bottom left corners must remain to the left of the top and bottom right corners, and the top left and right corners must remain above the bottom left and right corners after the transformation. If this condition is not met, $H_{i}$ is discarded. We also calculate the determinant of our normalized $H_{i}$. Experiments have shown that this value should not be close to zero [39].

\subsection{Results and Discussion}

We ran our pipeline on many of the image pairs provided in the architectural symmetry dataset [2] which contains images with a variety of visual discrepancies. This dataset includes paintings, drawings, and historical photographs matched to modern day images and several image pairs taken at very different times. Some image pairs are obtained under different lighting conditions, including changes in season and time of day, and have scale and perspective changes. The dataset includes ground truth homographies for aligning image pairs. We ran several tests on this dataset to deter- 
mine the effectiveness of the first version of our pipeline which builds upon a local feature matching technique such as SIFT. The first of these tests is shown at the top of Figure 2.13. The goal of these tests and Figure 2.13 is to show how we can increase the accuracy and robustness of a widely used feature matching technique with our contextual framework. This test consisted of stitching the images using the homography calculated from four different matching techniques. We show results for using traditional SIFT matches with a 0.7 distinctiveness ratio, SIFT matches with a 0.9 distinctiveness ratio, our ensemble features, and our ensemble features with our refinement pipeline. The contextual features identified in conjunction with our ensemble features are based on SIFT keypoints that have distinctiveness ratios between 0.7 and 0.9 . We use the first homography calculated with our pipeline since only one ground truth transformation is provided for each image pair. We have included both standard SIFT results to show that just increasing our search range to potential matches with ratios up to 0.9 does not account for the increased match pool and accuracy produced by our pipeline. The overall accuracy of using all SIFT matches with a distinctiveness ratio below 0.9 is very low.

For each of these four matching technique tests, we translate every pixel in $I_{1}$ to $I_{2}$ using both the ground truth homography and the first homography calculated using the method being tested. We compute the percentage of pixels that are transformed to the same coordinate. To allow for rounding errors and small inaccuracies in the ground truth data, we set a threshold on what the 2D distance between projected pixels can be to label a pixel as having been transformed correctly. This threshold is $0.6 \%$ of $\max \left(\right.$ image $_{\text {width }}$, image $\left._{\text {height }}\right)$ which is the same threshold used on 2D symmetrical transfer errors for identifying inliers in [48].

Our proposed ensemble feature compares favorably to SIFT across this dataset. From our tests, we also have determined that blindly raising the distinctiveness ratio allows too many inaccurate matches for RANSAC to find a correct homography 
between the image pairs and yields very unstable results. However, our approach for looking through these more ambiguous matches and considering contextual information appears to be a very valuable source of information. We increase the size of our match pool, making it easier to find a correct alignment, without letting in so much noise that RANSAC is overwhelmed by the outliers.

We also performed a test to observe what percentage of the keypoint matches identified in the different techniques are indeed correct. These results are shown in Figure 2.13 Center. From this chart we can see that, in general, adding in contextually verified matches increases the percentage of accurate correspondences in the pool of matches. In theory this should increase the odds of a correct aligning transformation being calculated using RANSAC. In conjunction with this, we also charted the number of each type of match that was used in Figure 2.13 Bottom.

In Figure 2.12, we show the precision-recall curves for four different image pairs in the symmetry dataset. These curves were obtained by increasing the distinctiveness ratio $(r)$ for accepting matches. $r$ increases from left to right. We show the curves for using just SIFT features, using our ensemble features, and using only our contextually-verified features. The ensemble feature contains SIFT features with ratios under $r$ and contextually-verified features found within a distinctiveness ratio range of $[r, r+0.2)$. The contextual curve shows only SIFT features that passed either the linear or HOG requirements for all matches with ratios under $r$. The contextual and ensemble features perform quite well under this metric. Combining the precision-recall measurements with those shown in Figure 2.13 demonstrates that the contextual information we propose using can complement local keypoint matching techniques very well.

In Figure 2.15, we also provide the image alignment accuracy results for each of the three versions of our pipeline. These consist of building on SIFT and creating an ensemble feature of SIFT and contextual descriptors, matching Harris-Laplacian 
corners with contextual descriptors, and matching our proposed Pseudo Corners with contextual descriptors. This chart shows the alignment accuracy using the initial set of matches from each of these methods and the accuracy after refinement is applied. We can see here that each of the images tested can be aligned very well using one of our techniques. Our contextual descriptor framework can be used successfully in combination with a variety of keypoint detectors.

Further tests of our contextual corner methods are shown in the supplementary material in which we used both our Harris-Laplacian-based method and Pseudo Corner method to align images in the png-ZuBuD dataset [6], the Stanford Mobile Visual Search dataset, and the dataset provided in [1]. Visual results for several of these image pairs are shown in Figure 2.2 and in the supplementary material. For these tests, we compute a homography using the matches calculated from both tested techniques and report the number of matches found and the average symmetrical transfer error [38] for all of the matches using the estimated homography. These tests show that we can obtain sufficient numbers of matches between image pairs with a wide variety of visual properties to find an aligning transformation. They also show, via their low average symmetrical transfer error, that the majority of the matches found agree with each other about the value of the solved homography matrix. This indicates that the matches found are structurally consistent.

To test our proposed feature detector, Pseudo Corners, we computed its repeatability scores on the Oxford Affine Covariant Region Detectors Dataset [3] which provides image sets demonstrating changes in perspective, lighting, scale, and JPEG compression. We compared the repeatability of our Pseudo Corners to that of SIFT and Harris-Laplacian. These values are shown in Figure 2.14. Our Pseudo Corners method tends to out perform SIFT and Harris-Laplacian on all of the image sets except "bark" and "wall". Given the nature of Pseudo Corners, which looks for the intersections of line segments, it is reasonable that it does not perform as well on 
these natural images.

We have also included several visual examples of image registration using our pipeline (Figure 2.2) for photographs from our own dataset, the symmetry dataset, and several other public datasets (see supplementary material). These image pairs present challenges including changes in season and content, lighting, scale, camera orientation, blur, and rendering styles. The runtimes to register many of these image pairs using our pipeline are also provided in the supplementary material. Both quantitative and qualitative comparisons of our method to other feature extraction and matching techniques including MSER, SIFT Flow [9], and local symmetry features [2] are presented in the supplementary material as well. 

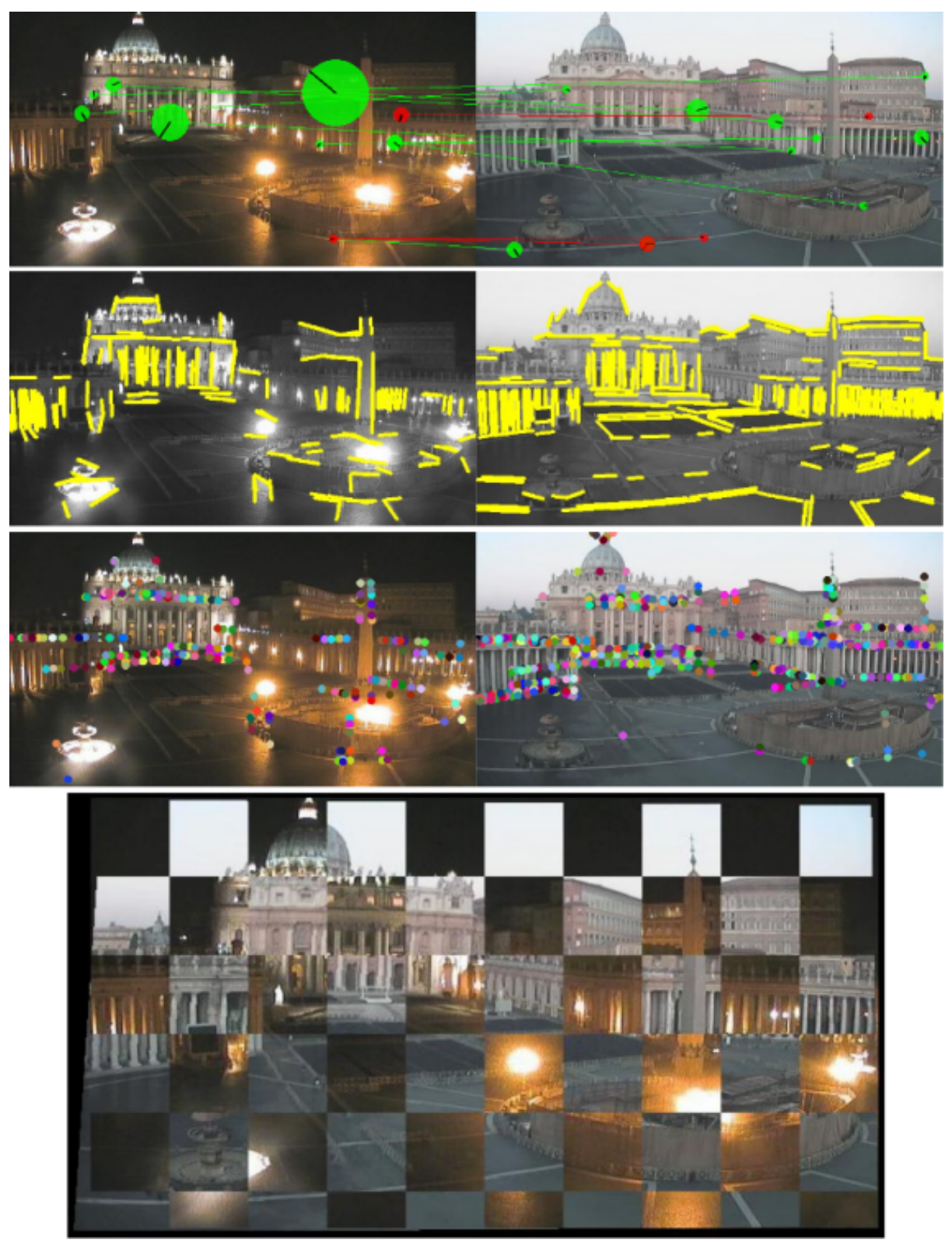

Figure 2.9: Using Pseudo Corners to align a difficult image pair, called Vatican. Top: The majority of the SIFT (green) and linearly-verified (red) keypoint matches for this pair are incorrect and cannot be used to align the images. No SIFT+HOG matches were identified in this example. The relative SIFT scales are represented by the size of the circles and the features' orientations are denoted by the black lines. 2nd Row: Line segments extracted from images (shown in yellow) that are used to find Pseudo Corners. 3rd Row: Pseudo Corners identified after intersecting line segments. Bottom: Image pair is aligned using the homography calculated from the matched Pseudo Corners. 


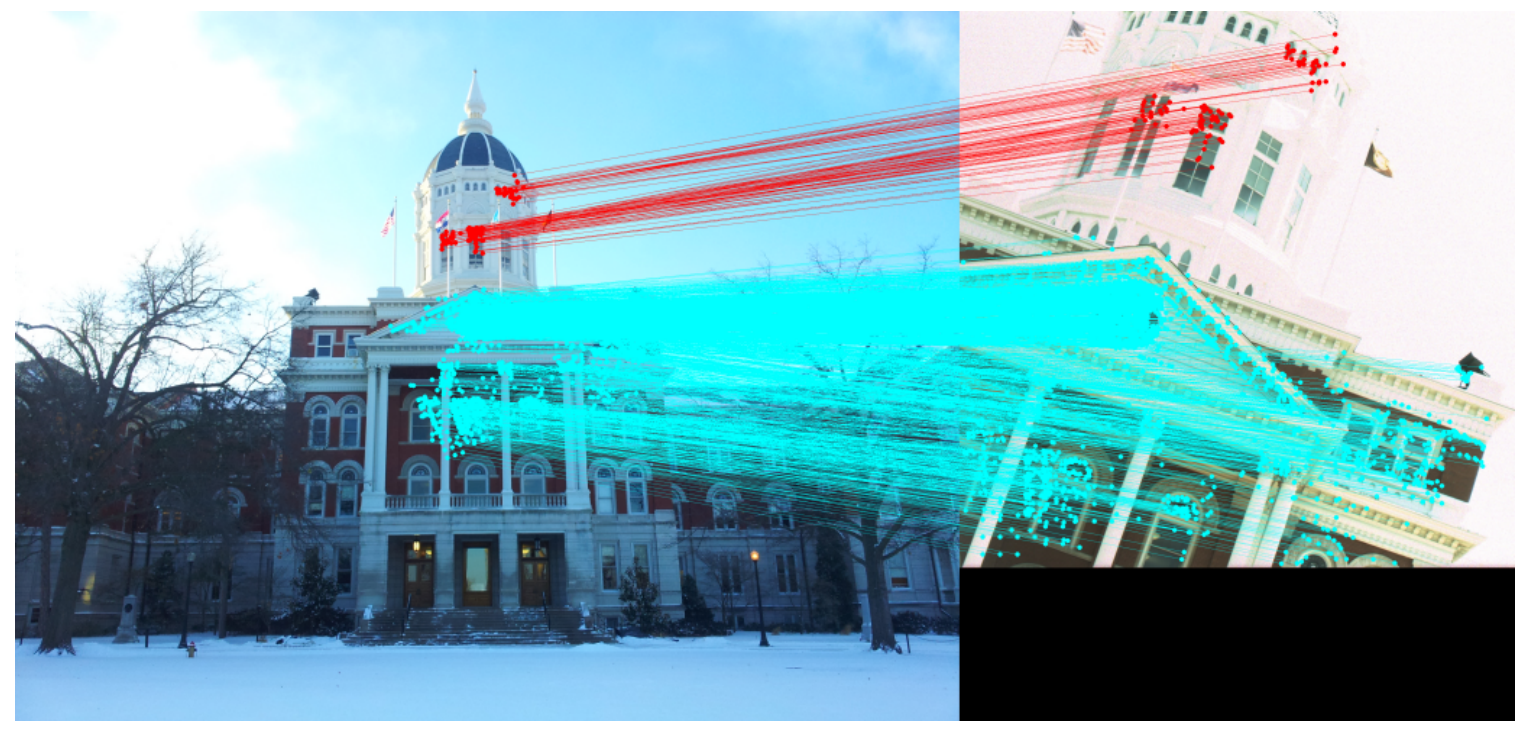

Figure 2.10: Matches identified using multiple homographies. Each color corresponds to a different homography. We can see how each homography tends to find matching points in different regions and planes of the image.
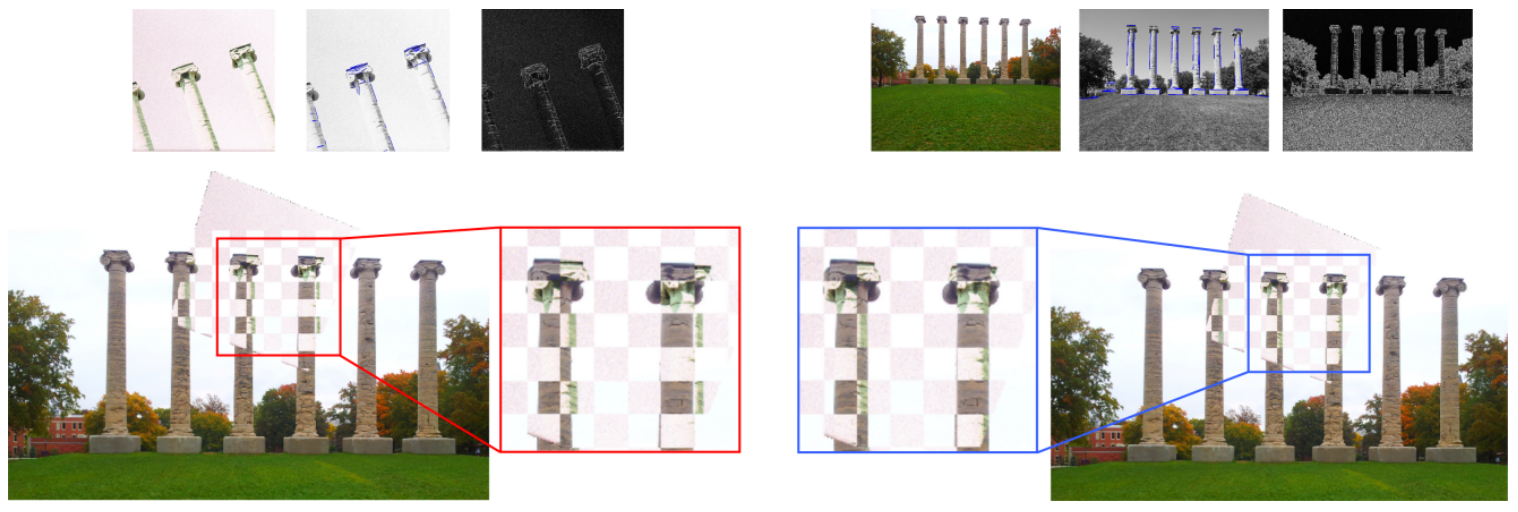

Figure 2.11: Using gradient information to refine homography. Top: Two original images and their edge images and gradient field images. We aim to align the edge and gradient field images as well as possible. Bottom Left: Stitching result before our refinement method is applied. Bottom right: Result after our refinement is applied. Bottom middle: Zoomed in views of the area where the alignment is corrected through refinement. 
Arch

Precision-Recall Curves for Image Pairs
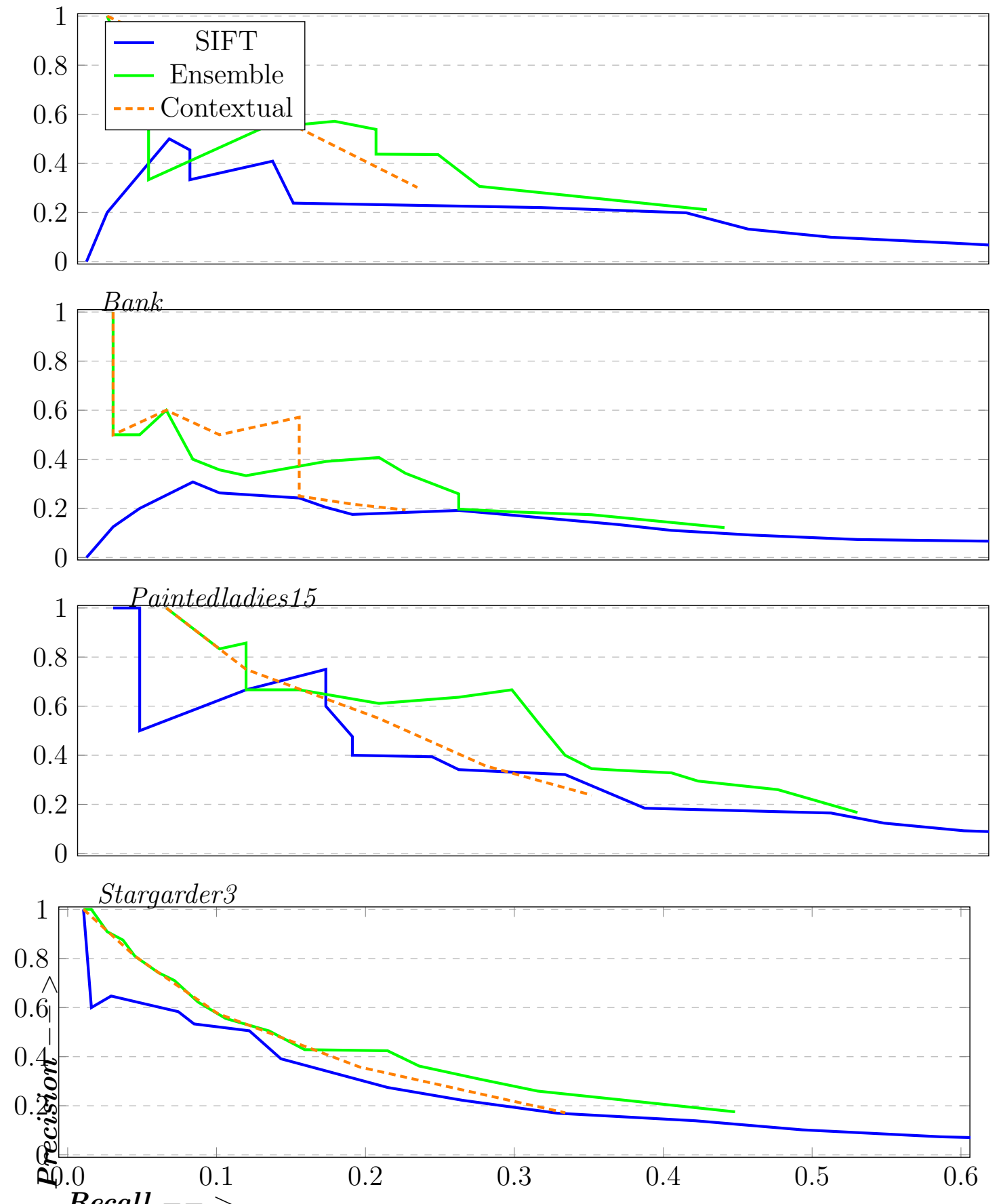

Recall - - >

Figure 2.12: Precision-recall curves for four different image pairs from [2]. Curves are provided for using SIFT matches, our ensemble feature, and just the contextually verified SIFT features identified using our method. Data points correspond to increasing distinctiveness ratios moving from left to right. 
Image Stitching and Keypoint Matching Measurements
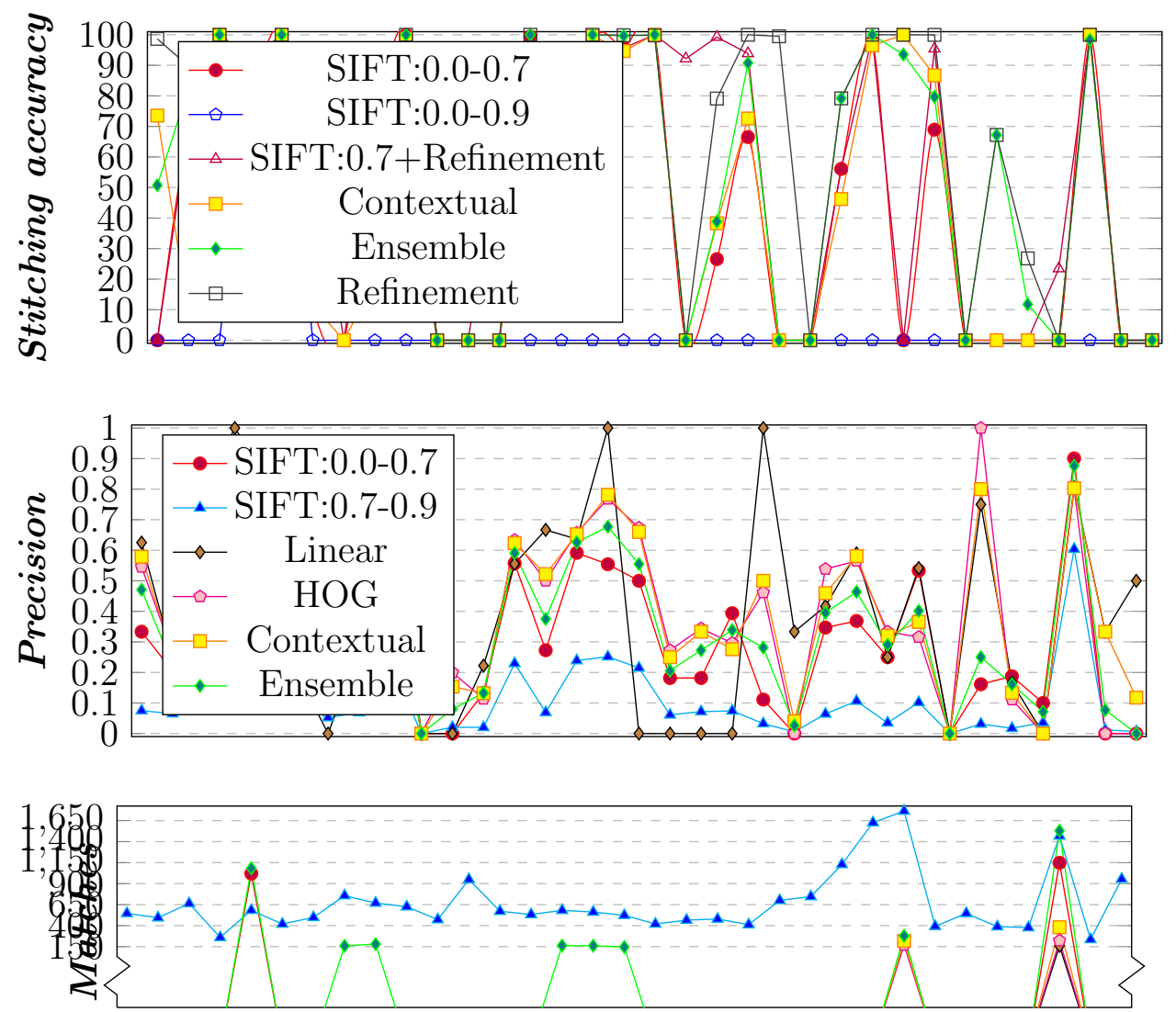

\$

Figure 2.13: Quantitative results for stitching image pairs. Top: Percentage of pixels in image plane that are correctly aligned after using solved homography. Center: Percentage of matches that are identified using different methods that are correct. Bottom: Number of matches identified using several different methods. 


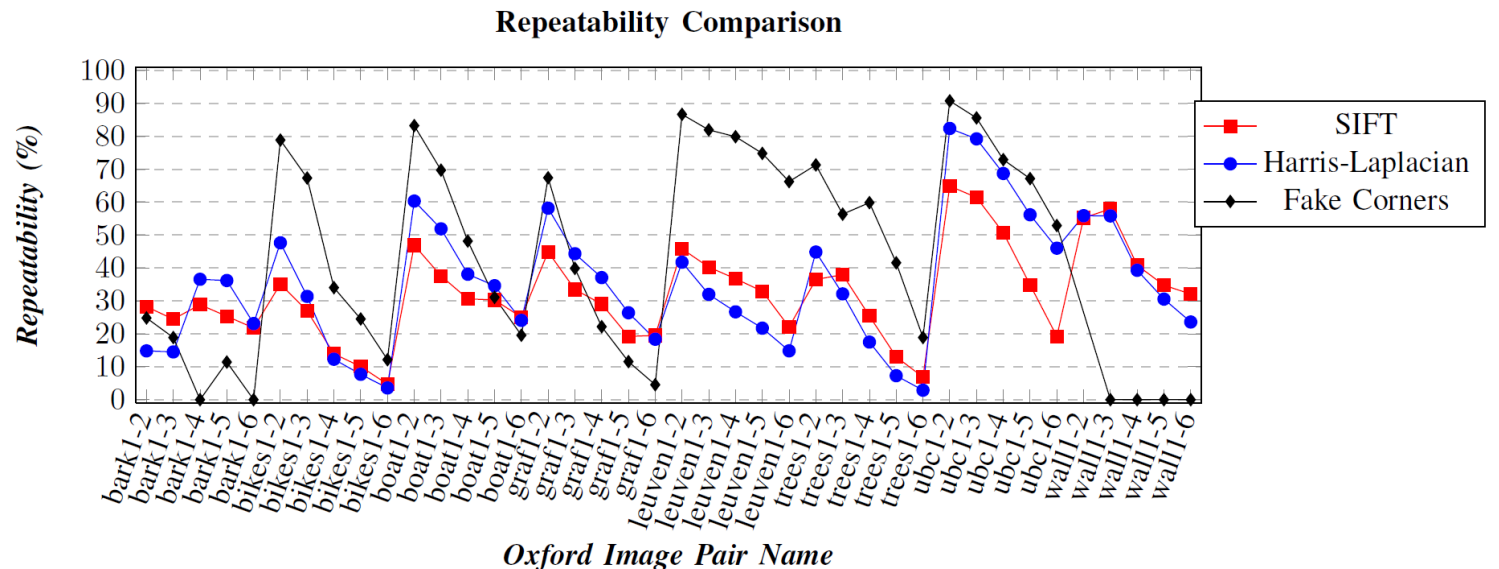

Figure 2.14: Repeatability measurements for SIFT, Harris-Laplacian, and Pseudo Corners on Oxford Affine Covariant Region Detectors Dataset [3]. The sets of images test the following changes in image properties: bark - scale and image rotation changes on textured scene, bikes - increased blur on a structured scene, boat - scale changes, graf - viewpoint changes, leuven - illumination changes, trees - increased blur on a structured scene, ubc - changes in JPEG compression, wall - viewpoint angle changes on textured scene. 


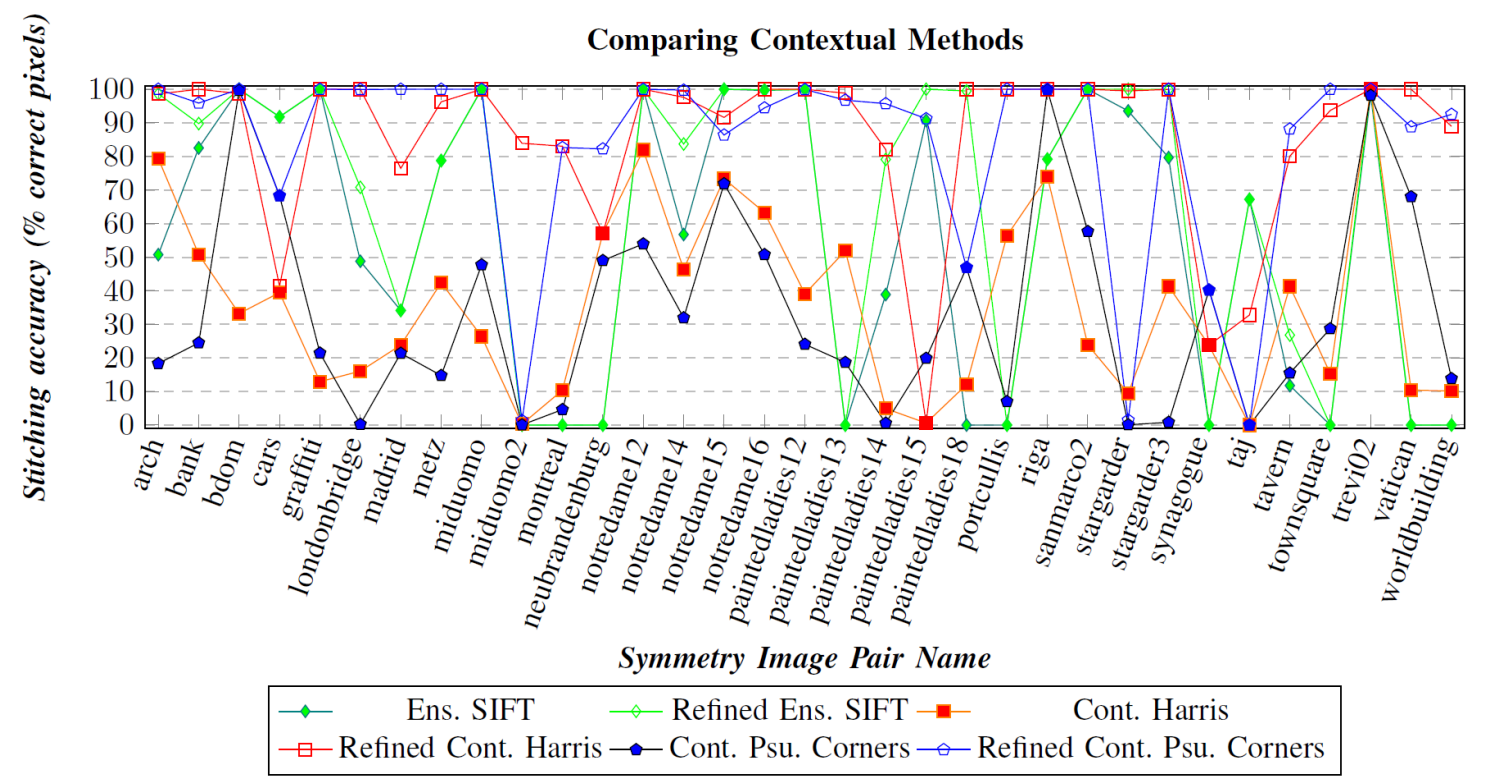

Figure 2.15: Comparing our contextual methods. Using the symmetry dataset [2], we show the alignment accuracy using our ensemble SIFT features before and after refinement, contextual Harris-Laplacian before and after refinement, and contextual pseudo corners before and after refinement. As mentioned in Section 2.1.3, ensemble features refer to the version of our method that uses an existing keypoint descriptor, such as SIFT, in addition to our contextual linear and contextual HOG information for matching. Contextual features use only our contextual descriptor for matching. The accuracy is measured in terms of the percentage of pixels in $I_{1}$ that are transformed to the correct location in $I_{2}$ using the homography estimated from the match set. 


\section{Chapter 3}

\section{D Matching Using Repetitive and Salient Features in Architectural Images}

\subsection{Literature Review}

Several groups have developed techniques to use different types of structural and highlevel information to guide image matching. The goals of these methods are generally to overcome pitfalls of locally or even regionally defined descriptors $[3,4,10,11,16$, 33] that are ambiguous in the presence of repetitive features. While locally defined descriptors can provide very accurate information that is invariant to scale changes and scene clutter, in certain cases they do not take into account large enough image regions to be truly distinct [13].

To make matching images with repetitive architectural patterns a more tangible task, researchers have explored using larger-scale properties such as feature symmetry $[25,49]$ and repetition to identify corresponding regions [5]. Hauagge et al. [2] search for horizontal, vertical, and rotational symmetries about various axes and scales across images. Self-similarities can be identified in patterns of colors, edges, 
and repeated visual elements by measuring how similar a small region is to parts of the larger surrounding region [26]. Wu et al. [50] match SIFT features within an image to identify repeating and symmetrical elements that occur at regular intervals and the boundaries between repetitions. Kushnir et al. [51] also match SIFT features across images to find repeating elements and use agglomerative clustering to group similar regions. This group presents two versions of their matching algorithm. Similar to [50], one version focuses on images where grids of periodic elements can be identified. The other handles cases where repetition exists in a less predictable fashion. Matches between non-repetitive features are identified and used to solve a transformation between an image pair. Chung et al. [52] use graph matching to globally align images of buildings that capture the same portion of a structure's facades. This work identifies repetitive MSER patches and constructs a semantic sketch of a building to match between highly varying viewpoints. Bansal et al. [53] also work to align images of buildings taken from highly different viewpoints such as aerial to ground level. Aligning images of high-rise buildings that have many repetitive elements and appearance differences due to view changes poses difficulties. They construct an embedding of a facade that captures how similar or dissimilar image regions are to their neighbors. This group uses the periodicity of a repeating element as an estimate of the scale of interest. This group's contribution of a "scale-selective self-similarity" can be extracted from any pixel in the image bypassing the need for a repeatable detector. PatchMatch $[54,55]$ provides a framework for matching patches across images by randomly assigning and searching for correspondences. This method uses a combination of searching through different scales, random sampling, and distance propagation to guide the matching process using a cooperative hill climbing strategy to find a patch's set of nearest neighbors. Ceylan et al. [5] construct grids of repetitive elements within images and limit an aligning transformation to be one of a discrete set of possible solutions that overlay different images' grids. 


\begin{tabular}{|c|c|c|c|c|c|}
\hline \multicolumn{3}{|c|}{ Step 1: Vertical Alignment } & \multicolumn{3}{|c|}{ Step 2 - Horizontal Alignment } \\
\hline $\begin{array}{l}\text { Identify } \\
\text { Representative } \\
\text { Patches }\end{array}$ & $\begin{array}{c}\text { Match } \\
\Rightarrow \text { Representatives } \\
\text { Between Images }\end{array}$ & $\begin{array}{c}\text { Compute } \\
\text { 1-D Vertical } \\
\text { Homography }\end{array}$ & $\begin{array}{l}\text { Compute } \\
\text { Intra-Image } \\
\text { Saliency }\end{array}$ & $\begin{array}{c}\text { Match Salient } \\
\text { Regions } \\
\text { Between Images }\end{array}$ & $\begin{array}{l}\text { Vote for } \\
\text { 1-D Horizontal } \\
\text { Homography }\end{array}$ \\
\hline
\end{tabular}

Figure 3.1: Pipeline of our proposed registration method. Our method has two main stages: vertical alignment and horizontal alignment. For vertical alignment, we perform dimension reduction, identify representative patches for each image, and use the matching representatives to compute a 1-D transformation that transforms the images to the same scale and aligns the pair along the vertical axis. For horizontal alignment, we compute the intra-image saliency and search for strong, salient matches along corresponding rows between images to vote for the correct displacement between the pair.

A different perspective for matching data that has repetitive elements is to find the salient regions in images and use these areas to guide registration. Machine learning techniques have been used to identify these types of unique regions in photographs, paintings, and 3D models $[1,27,28,56]$. These mid-level discriminative features ideally occur often enough across a dataset to be learned, but are different enough from the rest of the imagery to be clearly located in photographs and models with visual dissimilarities. Training a system to learn these features tends to be time consuming and computationally intensive. In this paper, we share a similar spirit of finding salient regions. However, unlike existing work that requires supervised training, our proposed salient region detection method is applied directly to and only on the images being matched and is unsupervised.

\subsection{Contributions}

Our main contributions to the area of architectural imagery registration include methods for:

1. aligning rectified images one dimension at a time, reducing the search space and increasing the image matching robustness,

2. computing intra-image saliency maps in an unsupervised manner with no train- 
ing set, and

3. directly accounting for image region saliency when quantifying keypoint match quality.

Our pipeline is designed to overcome several of the limitations and assumptions made in the work described in Section 3.1. We use a technique for identifying repetitive patterns in images regardless of the spatial distribution of similar elements and we are able to match images of repetitive facades that only partially overlap. We also remove the ambiguity in the feature space that is common in architectural imagery by collapsing repetitive elements into single representative patches. Finally, instead of only using either repetitive or salient features for matching, we take advantage of all the information available in images, and incorporate both types of features in our registration pipeline.

\subsection{Two-Step Approach}

The main steps of our registration methodology are identifying both repetitive and salient elements within images, using this information to first align image pairs vertically, and finally computing a horizontal alignment using a dramatically reduced and unambiguous search space. The first step of this process is solving for a 1-D affine transformation that encodes the relative scale change and vertical shift between images and matches rows to rows as shown in Figure 3.11c. The second step is determining a second 1-D transformation that represents the horizontal translation overlaying the image pair. Equation 3.1 shows the forms of these vertical and horizontal transformations $\left(T_{v}\right.$ and $\left.T_{h}\right)$ where $s$ is the scale change, $d_{y}$ is the displacement along the y-axis, and $d_{x}$ is the displacement along the x-axis. 


$$
T_{v}=\left[\begin{array}{cc}
s & d_{y} \\
0 & 1
\end{array}\right] \quad T_{h}=\left[\begin{array}{cc}
s & d_{x} \\
0 & 1
\end{array}\right]
$$

\subsubsection{Vertical Alignment}

We begin by performing dimension reduction and selecting one representative patch of each type of region in an image so that all the chosen representative patches are distinctive. This entails identifying portions of an image that are duplicates of each other and represent a repeating pattern as well as image portions that are truly unique. We use a combination of local and regional descriptors to accomplish this. Doing so helps overcome some of the inherit ambiguity of very-locally defined descriptors $[4,13]$ and increases our confidence as we categorize regions. Incorporating regional descriptors at varying scales also allows us to explore how different image regions may switch between repetitive and salient as the considered area changes.

To initially identify repetitive elements, we extract Psuedo Corners [4] which are sparse, repeatable local keypoints that are found at the intersection of line segments. Our design choice of using Pseudo Corners as anchor points for finding repetition and matching images is discussed further in Section 3.4.2. We extract multi-scale patches centered at each anchor keypoint and use local SIFT and regional HOG descriptors [4] to group repetitive elements that are centered on the same image row. For each group, we use the element that is most similar to the group's mean patch as the representative patch. Figure 3.2 provides a visual example of how we identify repetitive elements in an image and choose a group's representative patch. We use the $\mathrm{y}$-coordinate of these representative patches to solve for $T_{v}$.

When we match just the representative patches of groups (that may have either one or multiple elements), we can focus on matching unique areas without worrying 
about horizontal ambiguity. To actually match representative patches, we again use the SIFT descriptor at the center point of each patch and the HOG descriptor of the entire representative region. For each representative patch in $I_{1}$, we compare its SIFT descriptor to all of the representative patches in $I_{2}$. If the patch in $I_{2}$ with the most similar SIFT descriptor to the patch in $I_{1}$ also has Euclidean distances between the center points' SIFT descriptors and the patches' HOG descriptors below a threshold (we use 0.5), we label this as a group match. We consider all of the representative patches in $I_{1}$ and $I_{2}$ that were constructed at different scales in one round of matching. Figures 3.2 and $3.11 \mathrm{~b}$ show examples of matching groups between an image pair.

Using RANSAC [12], we randomly choose sets of two matching representative patches between the image pair to compute a series of potential $T_{v}$ 's. For each $T_{v}$, we check how many other representative patch matches have a symmetric transfer error [38] lower than $0.5 * w_{i n}$ Size $_{1}$ and choose the $T_{v}$ with the largest support. We then re-estimate $T_{v}$ using this set of inliers.

\subsubsection{Horizontal Alignment}

Since we are working with data containing repetition, especially images with patterns along the horizontal direction, we need to identify matching areas where we can be sure the correspondences are non-ambiguous to compute $d_{x}$. This is especially important because we do not require that our image pairs contain completely overlapping facades, so we can not rely on the relative arrangement of repetitive elements to determine the horizontal alignment. Figure 3.3 shows an example of the problem of relying on the relative arrangements of repetitive elements to align images. Our goal is to find matching pixels $\left(p_{1}\right.$ in $I_{1}$ and $p_{2}$ in $I_{2}$ ) that satisfy the following criteria:

1. $p_{1}$ and $p_{2}$ have very similar HOG descriptors

2. $p_{1}$ 's closest match within $I_{1}\left(q_{1}\right)$ is very dissimilar to $p_{1}$ 
3. $p_{2}$ 's closest match within $I_{2}\left(q_{2}\right)$ is very dissimilar to $p_{2}$

These conditions help us ensure that we find matching pairs of pixels that are both very similar to each other, but are distinct within their respective images, making the match distinct. We have two steps for identifying salient matches. The first is to compute an intra-image saliency map to identify distinct areas in a single image. The second step uses this information to compute the pairwise saliency of each match.

\section{Intra-Image Saliency Maps}

The goal of our intra-image saliency maps is to display how salient each pixel and its surrounding region in an image are compared to other regions in the image. Since at this point in our pipeline, for a given pixel in $I_{1}$, we are only looking for its match on a single row in $I_{2}$, we are only concerned with how similar each region is to all other regions centered on the same row. This reduces our intra-saliency computation by requiring that we only compare each region only to the other non-adjacent regions on the same image row. We consider regions whose HOG windows do not overlap to be non-adjacent. Equation 3.2 shows how we compute each pixel's saliency. We use $s_{p_{i}}$ to denote the saliency of a pixel, $p_{i}$, and its surrounding patch. $q_{i}$ is the pixel on the same row as $p_{i}$ whose surrounding HOG patch has the lowest $L^{2}$ distance to the HOG patch surrounding $p_{i}$ and is not adjacent to $p_{i}$. $i$ denotes the image index.

$$
s_{p_{i}}=\arg \min _{q_{i}}\left\|H O G\left(p_{i}\right)-H O G\left(q_{i}\right)\right\|
$$

Using this equation, we gauge the saliency of each pixel and its surrounding region by the distance between its HOG descriptor and its closest match on the same row. Figures $3.4 \mathrm{~b}$ and 3.11d show visualizations of the range of intra-image saliency values found in each image. We compute the saliency at multiple scales or HOG window sizes as shown in Figure 3.11d to consider the saliency of different image region sizes. 
The ranges shown in these figures are colored with a standard jet colormap where the minimum values in the image are assigned a blue color, the maximum values are assigned a deep red, and the values in between interpolate through blue, green, yellow, and red. The specific colors chosen for visualizing the range of values are arbitrary. We use them as a tool to easily study the numeric values of how salient each region in the image is. We can see in these examples how the more distinctive features and objects that are only seen once in an image stand out from the repetitive elements.

\section{Pairwise Match Saliency}

Once we know how salient each pixel is within its own image, we can use this information to gauge how confident we are in the saliency of keypoint matches found between the image pair. We define this value, $m_{s}$, in Equation 3.3. $H O G\left(p_{1}\right)$ is the HOG descriptor of a pixel, $p_{1}$, in $I_{1}$. We compare $p_{1}$ to the HOG descriptors of all pixels lying on its corresponding horizontal line in $I_{2}$. HOG $\left(p_{2}\right)$ is the HOG descriptor surrounding a pixel on this line that is most similar to $H O G\left(p_{1}\right)$. We enforce a bi-directional constraint on the matches here, meaning that $p_{2}$ is the best match for $p_{1}$ in $I_{2}$ and $p_{1}$ is the best match for $p_{2}$ in $I_{1} . \epsilon$ is a very small value to ensure both that $m_{s}$ does not go to infinity and that a perfect match that is also repetitive does not have a high saliency score.

$$
m_{s}=1-\frac{\left\|\operatorname{HOG}\left(p_{1}\right)-\operatorname{HOG}\left(p_{2}\right)\right\|+\epsilon}{\left\|s_{p_{1}}+s_{p_{2}}\right\|+\epsilon}
$$

If either $p_{1}$ or $p_{2}$ are elements of a repetitive pattern within $I_{1}$ or $I_{2}$, we cannot be confident that $p_{1}$ and $p_{2}$ are a unique and correct match. For this reason, we use the intra-image saliency scores $s_{p_{1}}$ and $s_{p_{2}}$ of both $p_{1}$ and $p_{2}$ respectively. We are confident in keypoint matches with high $m_{s}$ values since this indicates that the matching pixels have similar descriptors and that they are both distinct within their 
respective images, leaving little room for ambiguity in the pair match.

To distinguish the pixel matches that we are confident in from the ones that are either poor matches or ambiguous, we create an image visualizing the range of $m_{s}$ values in the same manner we used to visualize the intra-image saliency values. The color map of $m_{s}$ values for different image pairs are shown in as shown in Figures 3.4c and 3.11e. We apply Otsu thresholding [57] to identify the "foreground" pixels in this image, or those whose saliency values stand out from the rest of the image. Figures 3.4d and 3.11f show the saliency match fields we are left with after applying this thresholding. We choose to use Otsu thresholding since it is an automatic method that is adaptable to different types of images and does not require that we set one threshold value for all datasets.

We use the pixel matches remaining in the pairwise saliency maps after thresholding to vote for $T_{h}$, with each match voting for a potential $d_{x}$ value. Each match's vote is weighted by it's $m_{s}$ score so that the most salient matches have a stronger voice in choosing the horizontal alignment.

We can encounter a potential obstacle during the horizontal shift voting stage if the scene in the image actually contains multiple planes that cannot be represented by a single transformation. For instance, as shown in Figure 3.11h., if an object, such as a tree, is in front of the main building being photographed, one transformation might align the two views of the tree captured between images, while a second transformation will align the building of interest. Since, in general, foreground items like this also tend to be salient, we may get a large number of pixel matches voting for the shift to align the salient foreground as well as a strong support for aligning the salient features on the more repetitive building facades.

To handle this scenario, we consider several different potential $d_{x}$ values. We have observed that the horizontal shift that aligns the salient foreground object does not also align the repetitive elements on the facade of interest, except by coincidence. 
Using this observation, if multiple $d_{x}$ values have strong support, we choose the one that best aligns the repetitive facades in the images. We have already identified the

image regions showing building facades when we extracted and matched groups of repetitive elements in Section 3.3.1.

To decide how many $d_{x}$ values have strong support, we consider all shifts that have at least $50 \%$ of the number of votes as the shift with the most votes. We use each of these shifts to project all the members from the repetitive groups extracted at the beginning of our pipeline in $I_{1}$ to $I_{2}$ and vice versa. For all of the members that are projected within the matching image's plane, we maintain a counter. The counter is incremented every time a repetitive element is projected onto the location of a member of its matching group. If there is no matching group member at an element's projected location, the counter is decremented. After projecting all the group members between images, we divide the counter value by the number of group members that were projected within their matching image's boundaries. We choose the horizontal shift which has the largest counter score. An example of using this criteria to choose the horizontal shift that best aligns a building facade with salient objects in front of it is shown in Figure 3.11.

\section{Full 8 Degree of Freedom Transformation and Refinement}

At this point we have two 1-D transformations that can be merged into one $3 \times 3$ homography that encodes the scale change between the image pair and the translations along the $\mathrm{x}$ and $\mathrm{y}$ axes. If both images have been perfectly rectified, this is all the information we need to align them. However, since we are working with realworld data and applying an automatic rectification algorithm to large sets of images, there is naturally still some warping and distortion in the rectified results. To handle these effects, we can switch to a traditional 8 degree of freedom (DOF) homography at the end of our pipeline, using the original two 1-D transformations as an initial 
alignment estimate. We refine our transformation [4] using the iterative closest point algorithm [47], switching to a full homography in the process.

\subsection{Evaluation and Discussion}

\subsubsection{Experimental Setup}

We have evaluated our pipeline on the $\mathrm{ZuBuD}$ building dataset [6] and two datasets containing symmetrical elements in urban scenes $[2,5]$ which we will refer to as the SymFeat dataset and the SymUrban dataset respectively. All of these datasets consist of a number of photographs of buildings under changing photographic conditions. In the $\mathrm{ZuBuD}$ dataset, there are five images of each building with each image varying in some aspect such as perspective, scale, or rotation. We used the software provided by [58] to rectify the images in the $\mathrm{ZuBuD}$ and SymUrban datasets. The majority of the images in the SymFeat dataset are already rectified and front facing. For the $\mathrm{ZuBuD}$ dataset, using the images that were automatically rectified correctly, we have 716 pairs of images for testing. The SymUrban dataset consists of 9 image sets, each focused on a single building. There are around 30 photos of each view. From the subset of images that were correctly rectified, we chose about 50 image pairs that represented all of the view changes presented by the dataset. We use selected images with the image pairs in the SymFeat dataset that had reliable ground truth information to test our method to see if we can overcome some of the common challenges of aligning urban images. We ran SIFT, the contextual regional matching method described in [4] on all these datasets. We chose these methods for comparison because both have been shown to be robust under changes in photographic conditions, but due to their relatively local nature (compared to using some sort of grouping and image abstraction), can be confused by repetition ambiguity in images. We also com- 
pare our method to the symmetrical feature-based methods described in [2] to look at other approaches specifically designed for urban imagery. A list of the names and thumbnails of the SymFeat and SymUrban images we used as well as the especially challenging images from the $\mathrm{ZuBuD}$ dataset are provided in the Supplementary Material. To obtain ground truth for the $\mathrm{ZuBuD}$ and SymUrban images, we manually selected a set of four matching points between image pairs to compute an aligning homography. We use these ground truth homographies to quantitatively evaluate our results in Figures 3.5-3.9 and Table3.1.

\subsubsection{Results}

We ran a variety of tests to evaluate both different design choices in our pipeline and how they build on each other as well as the overall effectiveness of our proposed methodology. Our first set of tests looks at the contribution of breaking down the transformation estimation into two steps, focusing on aligning one dimension at a time. We next look at how matching accuracy increases as we progress through the steps of our pipeline, adding in the intra-image saliency and pairwise match saliency computations to the two-step framework. Included in our results is also an investigation of how the type of anchor point used for vertical matching impacts our results.

For all of our alignment accuracy tests, we compute a transformation matrix for each tested method. We translate every pixel in $I_{1}$ to $I_{2}$ using both the ground truth homography and the test transformation. We compute the percentage of pixels that are transformed to the same coordinate. To allow for rounding errors and small inaccuracies in the ground truth data, we set a threshold on what the 2D distance between projected pixels can be to label a pixel as having been transformed correctly. This threshold is $0.6 \%$ of $\max \left(\right.$ image $_{\text {width }}$, image $\left._{\text {height }}\right)$ which is the same threshold used on 2D symmetrical transfer errors for identifying inliers in [59]. 
After running these tests on the full datasets, we take the average values and report them in Figures 3.5-3.9 to provide an overview of the trends in the results. The Supplementary Material contains more detailed results and shows the evaluation for individual image pairs. In the Supplementary Material, since it would be unintuitive to show quantitative results for the over 700 image pairs from the $\mathrm{ZuBuD}$ dataset, we focus on about 50 image pairs that were identified as especially challenging because of the amount of architectural repetition present in these specific scenes. These images pose a number of problems for state-of-the-art techniques and we show how our pipeline can overcome these challenges. In the following tables, however, since we are showing average scores for each method on each dataset, we have included the results for the full $\mathrm{ZuBuD}$ dataset. The additional $650+$ images included from the $\mathrm{ZuBuD}$ results in the main paper do not necessarily contain repetition or any specific challenges for the state-of-the-art methods. Many of the buildings in these additional images have different types of features such as curved walls and domes that prevent the full building facades from being correctly rectified. Many of these images also do not require or fit the repetition identification and two-step alignment methods described in this and other repetition identification papers. Therefore, we encourage the reader to also study the results in the Supplementary Material that focus on the types of images this work is intended to benefit.

\section{Two-Step and Saliency Computation Evaluation}

We begin by performing tests to study the usefulness of our proposed two-step method for image alignment. We show that by aligning a single dimension first, instead of registering the full coordinate systems of an image pair, we can achieve a higher accuracy along that dimension. First, we aligned the test data using traditional SIFT matches with the 8-point algorithm [38] and RANSAC [12] to compute a full 8 degree of freedom homography. We then check the 1-D error of the matches along the y-axis. 
Then we use the y-coordinates of the SIFT matches to compute a 1-D alignment (as shown in Equation 3.1) and again check the error along the y-axis. Our third test is to check the accuracy along the y-axis of the 1-D transformation computed using the grouping method described in this paper. Table 3.5 shows the results of these tests on our three datasets. Both of the 1-D alignments generally yielded better results than the traditional 8 DOF computation across the test data. The benefit of our grouping method over both of the SIFT-based tests is seen especially on image pairs that have large scale, rendering, or lighting changes such as those encountered in the $\mathrm{ZuBuD}$ and SymFeat datasets.

Next, we look at what happens when we get to the second stage of the two-step approach. Table 3.6 shows results after using the SIFT-based and grouping-based techniques described above in combination with different methods for determining the horizontal alignment. We first align the images vertically (using SIFT features or grouping features) and then match SIFT features only along corresponding rows. In both tests, distinctive SIFT matches are used to vote for a horizontal alignment. We can see that this minimal version of the two-step approach tends to outperform the traditional technique of solving a full 8 DOF matrix on challenging, repetitive images. We also show the results of using our full pipeline, consisting of the two-step approach in combination with the intra-image saliency and pairwise match saliency computations. The general trend is that the basic two-step approach outperforms traditional SIFT matching and our full pipeline outperforms the basic two-step approach, showing the strength of our full pipeline. These results are shown in Table 3.6.

\section{Overall Pipeline Evaluation}

To further test the overall effectiveness of our full pipeline, we compute transformations for aligning the test image pairs using our pipeline, SIFT keypoint matches, contextual keypoint matches using the method discussed in [4], and symmetry-based 
features as described in [2]. Following the symmetry-based features paper [2], we tried matching the images using only symmetry descriptors and symmetry and SIFT descriptors concatenated. The alignment accuracies for these methods are shown in Figure 3.7 Top. We also look at the accuracy of the keypoint matches obtained with each of the first three test methods, the number of matches obtained with each method, and the percentage of these keypoint matches that are correct. Figure 3.9 shows the number of matches we obtain for each method and Figure 3.8 shows the precision of these matches. We can see from all of these results that our proposed pipeline tends to outperform the comparison methods. We do have several cases where the refined result has a significantly higher accuracy than the pre-refined result. Often times in these cases, the rectified images still have a small amount of warping which is not captured in our 1-D homographies. It takes the full 8 DOF transformation to model these distortions. Given the fact that our horizontal alignment stage takes into account dense matches in salient regions of the image pairs, as we saw in Figures 3.4 and 3.11, our pipeline also tends to have a dramatically higher number of keypoint matches in the end than our comparison methods. Despite the high number of matches we consider, they are comparably more precise to matches from other methods.

We show several visual examples of the result of our pipeline for qualitative evaluation in Figure 3.10. The images shown here are a sampling of the different types of data we handle. These image pairs have varying ratios of salient and repetitive regions. Several of the pairs also have changes in rendering style, time captured, scale, and lighting, all image characteristics that are known to cause challenges for many traditional keypoint matching methods [10]. Our method is able to handle many of these problems on top of the issues presented by repetitive data. 


\section{Table 3.1: Repeatability Comparison on ZuBuD Dataset}

\begin{tabular}{c|c|c} 
Pseudo Corners & SIFT & Harris-Laplacian \\
\hline 28.645 & 13.803 & 10.885
\end{tabular}

\section{Anchor Keypoint Evaluation}

To identify repetitive and salient regions for initial matching, as was discussed in Section 3.3.1, we need anchor keypoints to use for searching for and comparing regions throughout an image. There are a number of feature extraction methods that can be used for this purpose such as SIFT or Harris-Laplacian corners. Since most architectural images have abundant linear features that capture a great deal of a building's structure, we take advantage of this information to find anchor keypoints. We intersect line segments extracted from these images to estimate corner locations, which we refer to as Pseudo Corner keypoints [4].

To test the usefulness of Pseudo Corners in our pipeline, we computed its repeatability scores on the images we used for evaluation from the $\mathrm{ZuBuD}$ dataset. We compared the repeatability of our Pseudo Corners to that of SIFT and HarrisLaplacian. These values are provided in Table 3.1 which shows that our Pseudo Corner detector tends to out perform SIFT and Harris-Laplacian on our test images. Given that Pseudo Corners are based on line segments, it is very well-suited for architectural imagery. Since it tends to be more repeatable on this type of data, we chose to incorporate it in our work to help increase the chances of extracting groups for matching that will exist in both images. However, depending on the type of data being used and the designers' preferences, other feature detector may be used in its place with the rest of our pipeline being run exactly the same. To demonstrate this, we also tested out our pipeline on the $\mathrm{ZuBuD}$ dataset using SIFT features as the anchor keypoints for grouping. The result of doing so is shown in Figure 7 with the labels "Repetition SIFT" and "Refined SIFT" in the Supplementary Material. 
Table 3.2: Average Runtimes in Seconds and Number of Features for Stages of Our Pipeline on ZuBuD Dataset

\begin{tabular}{c|c} 
Pipeline Steps & - \\
\hline Average Image Dimensions & $628 \times 457$ \\
\#ine Segment Extraction (Section 3.4.2) & 0.5115 \\
\#seudo Corner Extraction (Section 3.4.2) & 1313 \\
\# Pseudo Corners & $\mathbf{1 . 2 8 3 8}$ \\
SIFT Descriptor Extraction (Section 3.3.1) & 566 \\
HOG Extraction (Section 3.3.1) & $\mathbf{8 . 2 6 2 5}$ \\
\# HOG Patches & 3.9168 \\
Repetition Identification (Section 3.3.1) & 141493 \\
\# of Groups & $\mathbf{1 1 . 9 1 5 5}$ \\
Group Matching (Section 3.3.1) & 259 \\
Vertical Alignment (Section 3.3.1) & $\mathbf{8 . 1 4 4 9}$ \\
Intra-Image Saliency Computation (Section 3.3.2) & $\mathbf{1 7 . 5 6 8 4}$ \\
Saliency Match Computation (Section 3.3.2) & $\mathbf{1 1 . 9 8 3 1}$ \\
Horizontal Alignment/Voting (Section 3.3.2) & $\mathbf{0 . 0 1 6 5}$ \\
Refinement (Section 3.3.2) & $\mathbf{5 . 9 6 2 8}$
\end{tabular}

\section{Runtime}

Table A.4 shows the average runtime of our pipeline on the tested $\mathrm{ZuBuD}$ images.

These images were matched on an Acer Laptop with $6 \mathrm{~GB}$ of memory and a $2.2 \mathrm{GHz}$

Intel Core i7 Processor running Ubuntu 12.04. 


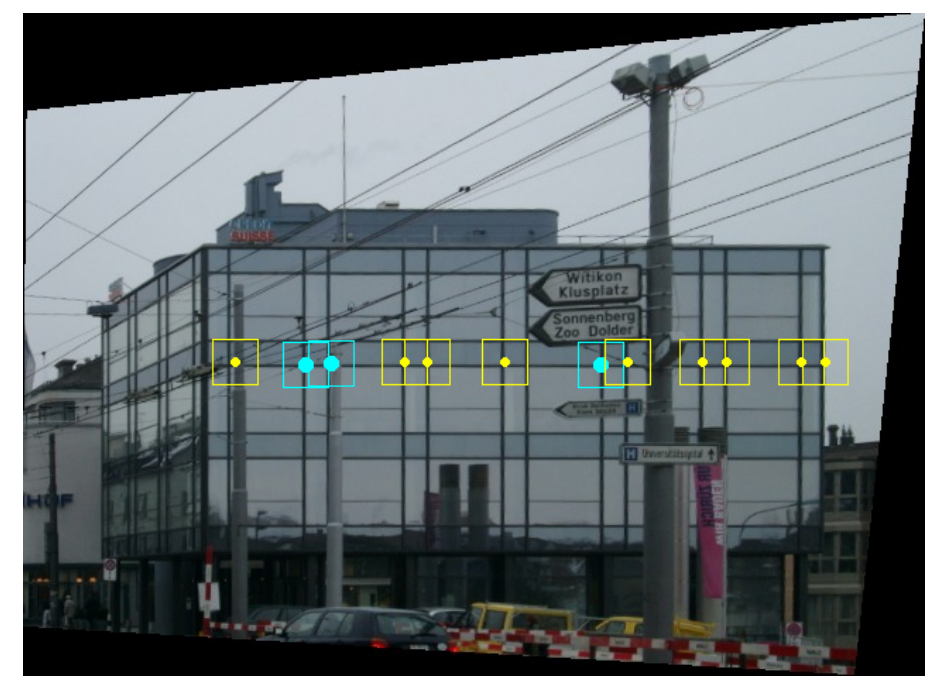

a.

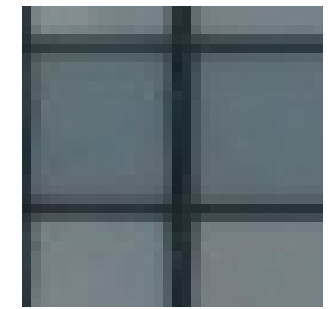

b.

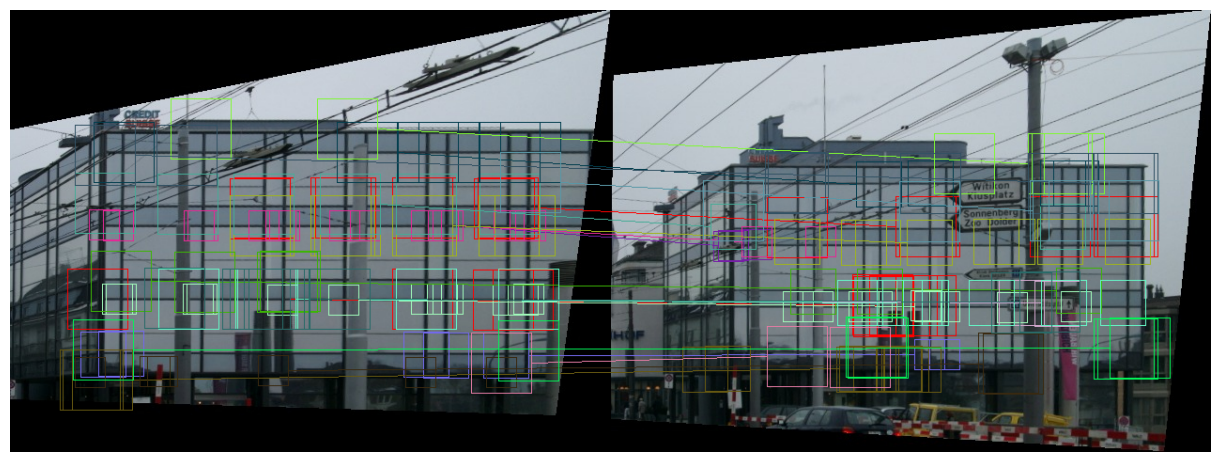

c.

Figure 3.2: Identifying groups of repetitive elements and matching groups. (a.) Similar patches are identified along a row of an image. Teal boxes surrounding a larger dot show the matching anchor keypoints. Yellow boxes surrounding a smaller dot show the dense features that were matched to the anchor keypoint for the row. For this example, we use Pseudo Corners as anchor keypoints as is discussed in Section 3.4.2. (b.) The chosen representative patch of the group. This patch is most similar to the average of all the elements contained in the group. Note that sometimes in architectural designs, the repetitive elements may not occur at regular intervals as shown in this example. Our method is designed to search for repetition regardless of the regularity or irregularity of the interval. (c.) Subset of the corresponding groups after computing a 1-D transformation are highlighted in matching colors. Every element of the groups are displayed. 


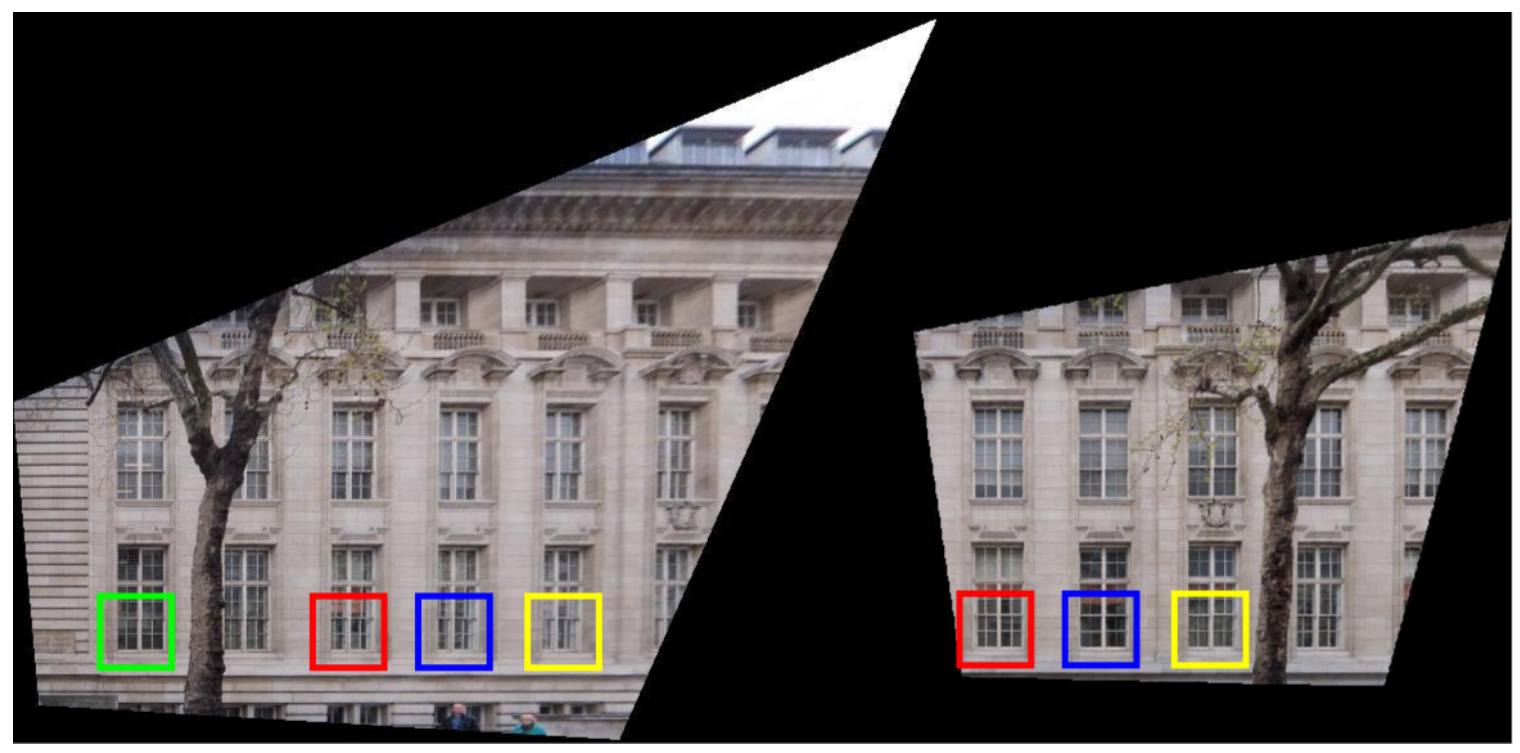

Figure 3.3: Motivation for searching for salient matches for horizontally aligning images. The highlighted image patches in the above image pair shows a set of matching repetitive elements. If we choose the horizontal alignment that maximizes the number of overlapping elements, we will have the wrong image pair transformation in situations like this where the images do not fully overlap. The highlighted patches are colored according to which patches will match if we try to maximize the number of overlapping patches. This is why we use our reduced search space (after vertical alignment) to identify distinct areas and salient matches to choose potential horizontal alignments. 

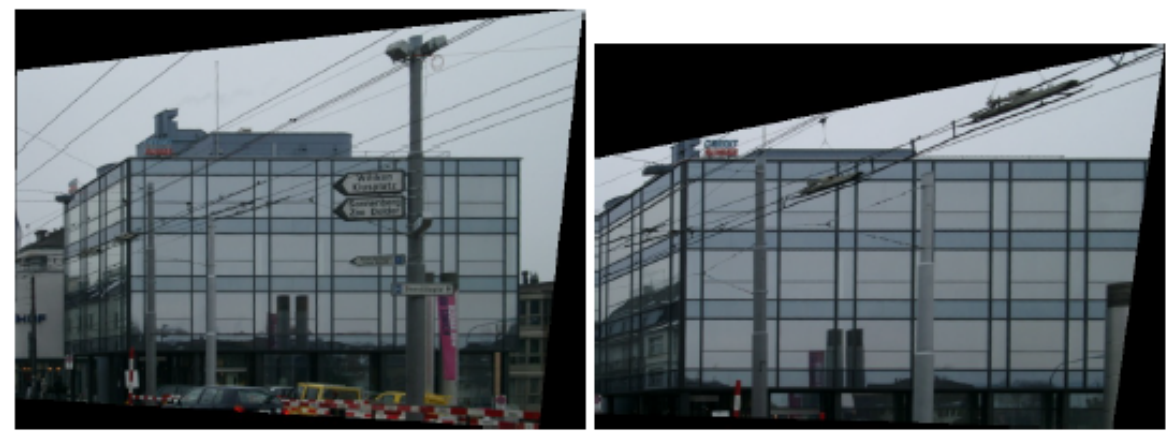

a.
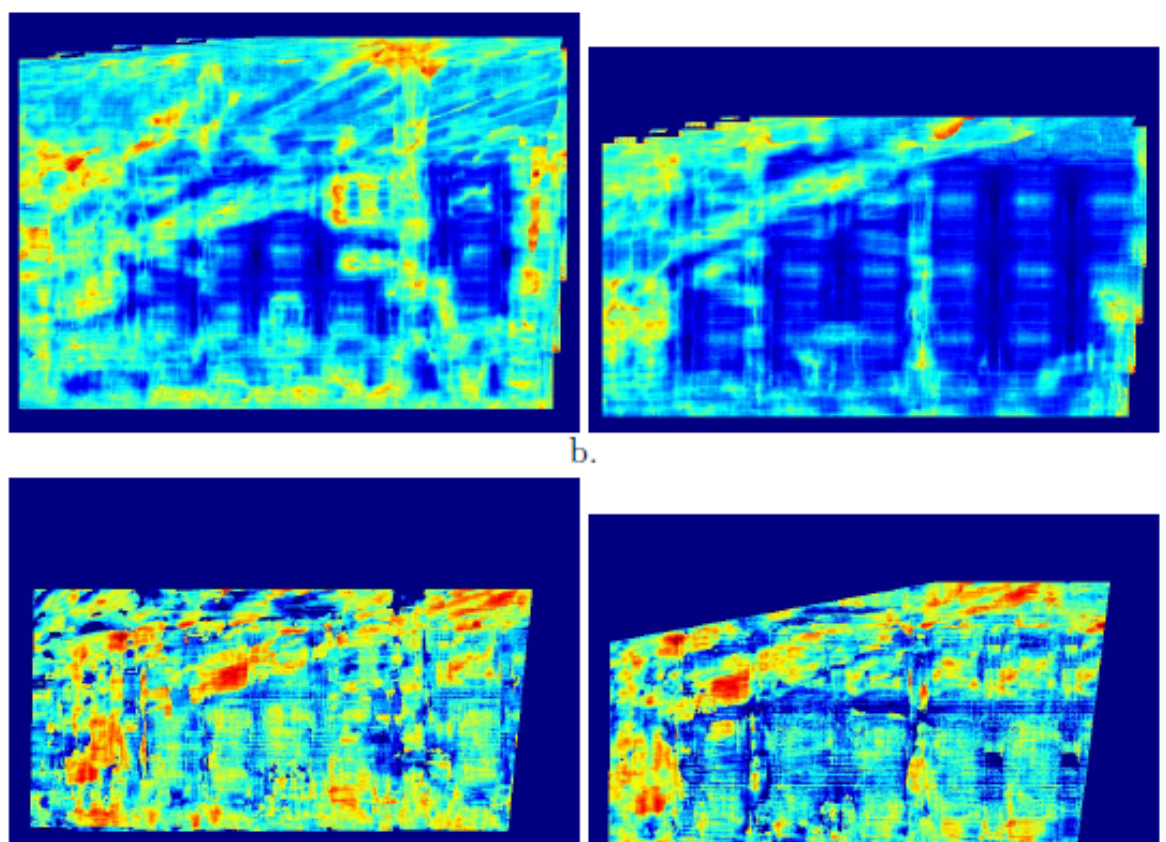

b.

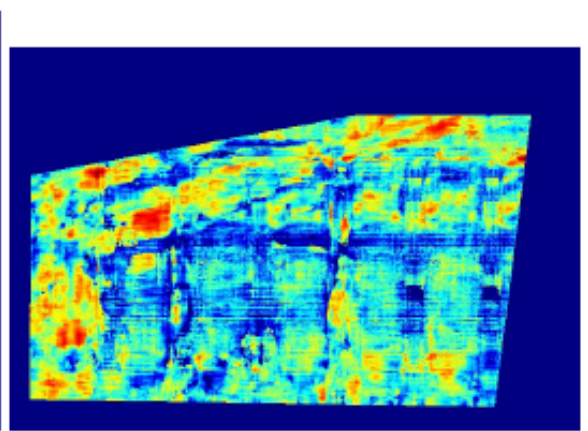

c.
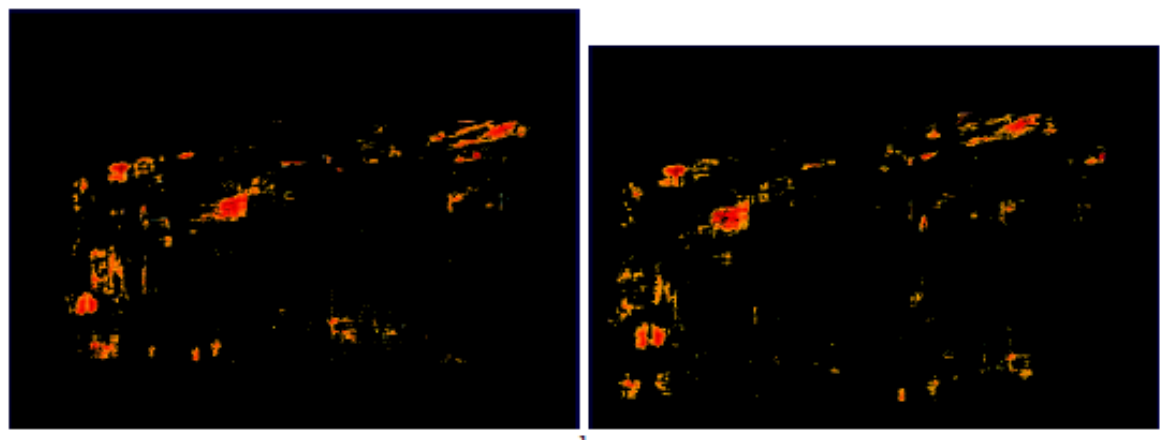

Figure 3.4: Visualization of match saliency equation. (a.) Original images being matched. (b.) Intra-image saliency map of each image. More salient areas appear in red and more repetitive areas appear in blue. (c.) Color fields of match saliency scores shown from the perspective of both images. (d.) Result after applying Otsu thresholding. All keypoint matches that are contained within the remaining colored regions are used to vote for a hortizontal alignment. 


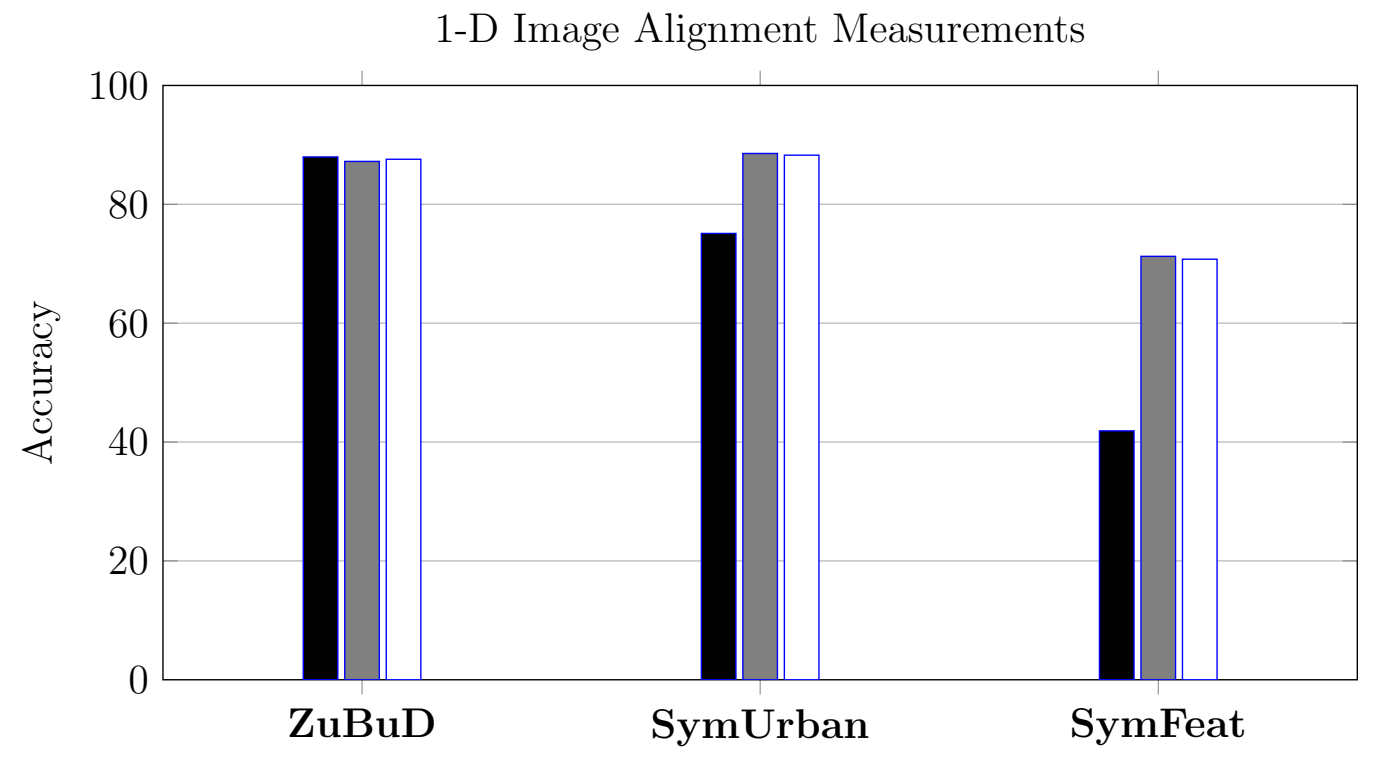

IITraditional SIFTL"1-D SIFTLu1-D Grouping

Figure 3.5: Accuracy measures for aligning images vertically, which is the first stage of our two-step pipeline. We show the results on our three datasets of using the traditional SIFT method, only the y-coordinates of the SIFT matches (1-D SIFT), and using our proposed method for vertical alignment (1-D Grouping). 


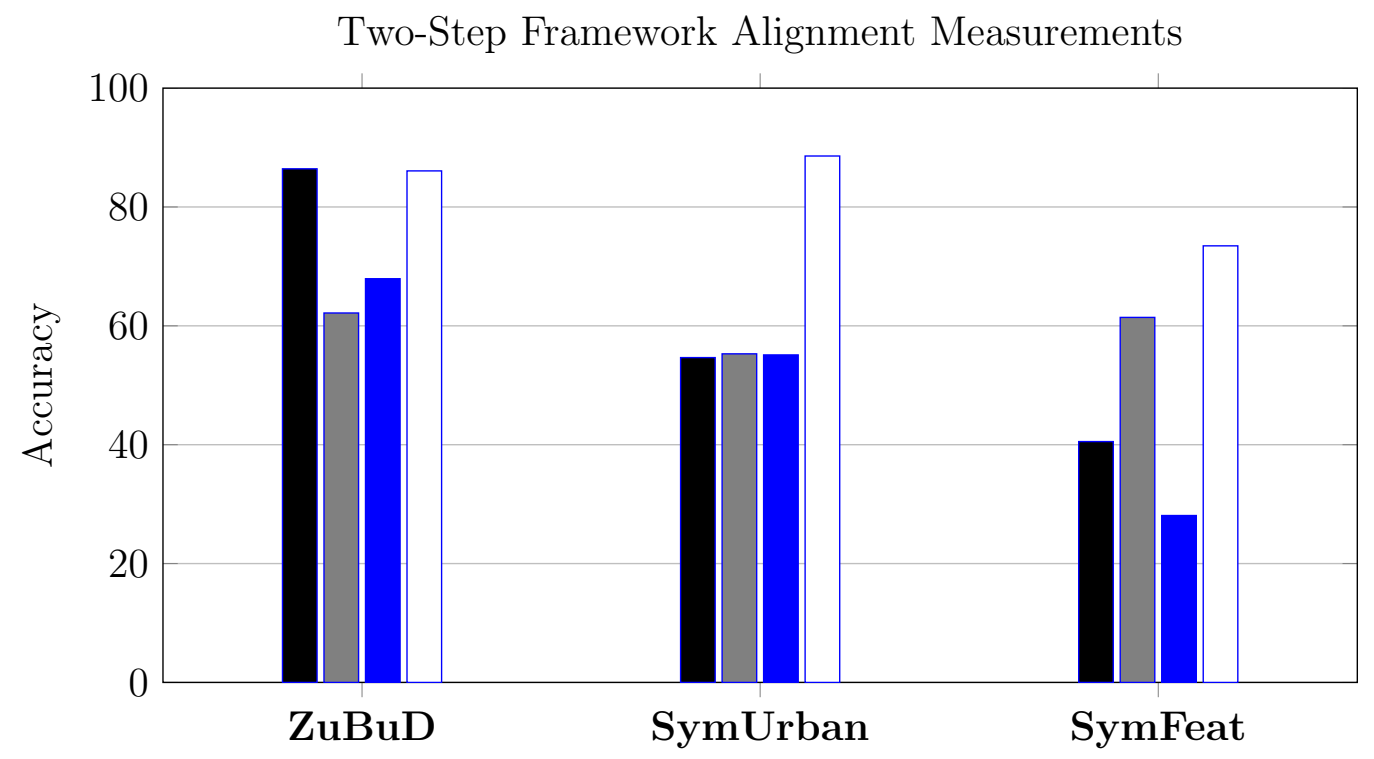

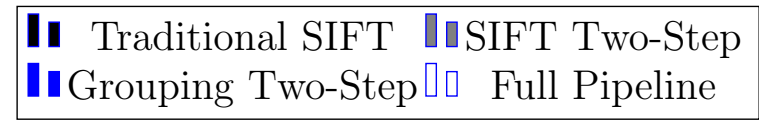

Figure 3.6: Accuracy measures for aligning images both vertically and horizontally. We show the results on our three datasets of using the traditional SIFT method, using SIFT matches to align the images first vertically, then horizontally (SIFT Two-Step), our proposed method for vertical then horizontal alignment (Grouping Two-Step), and our full pipeline.

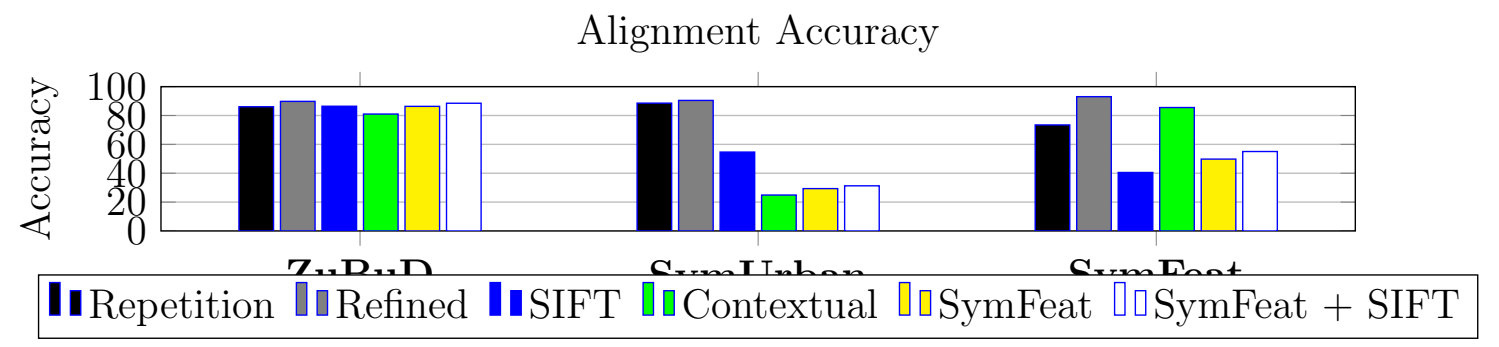

Figure 3.7: Accuracy measures for fully aligning images using our complete pipeline compared to state-of-the-art methods. We show the results on our three datasets of using our proposed method (Repetition), our proposed method with refinement (Refined), the traditional SIFT method, the contextual method described in [4], the two vartions of the symmetry-based features method described in [2]. 


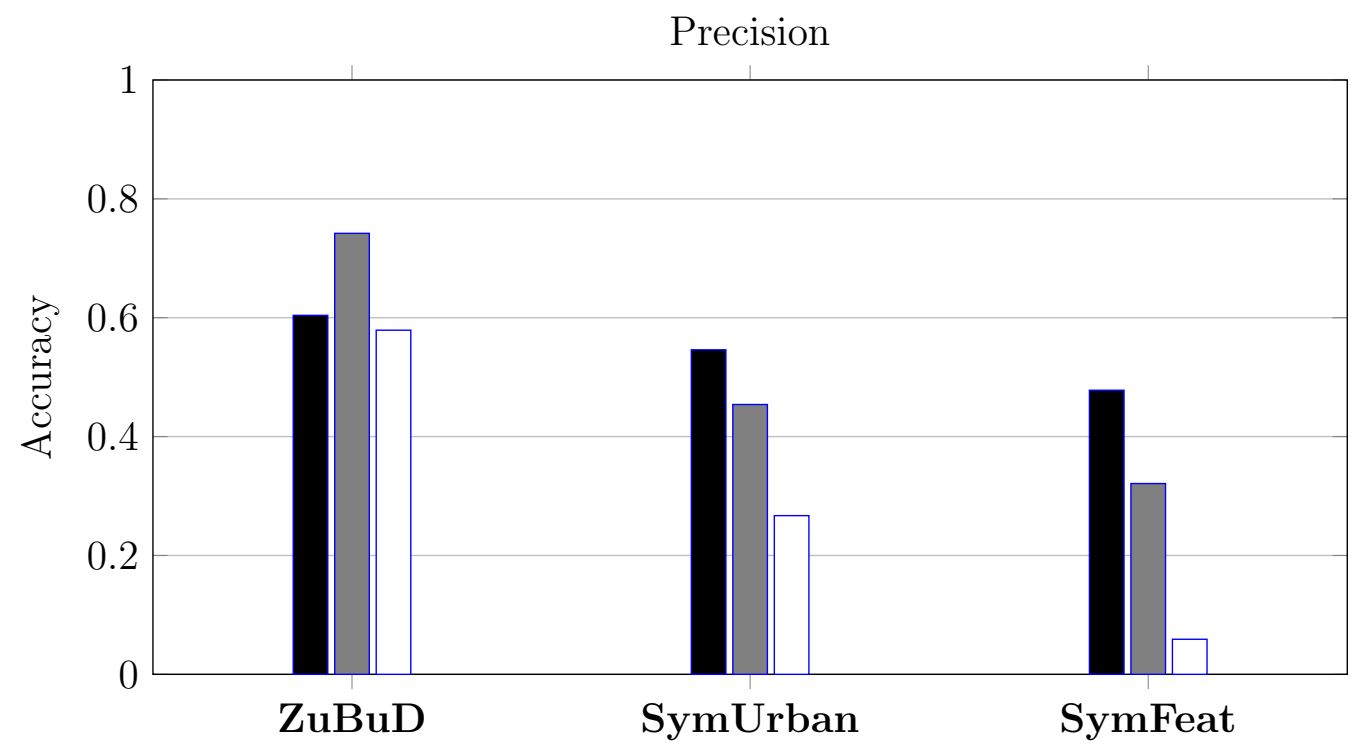

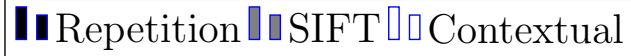

Figure 3.8: Precision measurements of matches found in the keypoint matching methods tested and shown in Figure 3.8. We show the results on our three datasets of using our proposed method (Repetition), the traditional SIFT method, and the contextual method described in [4].

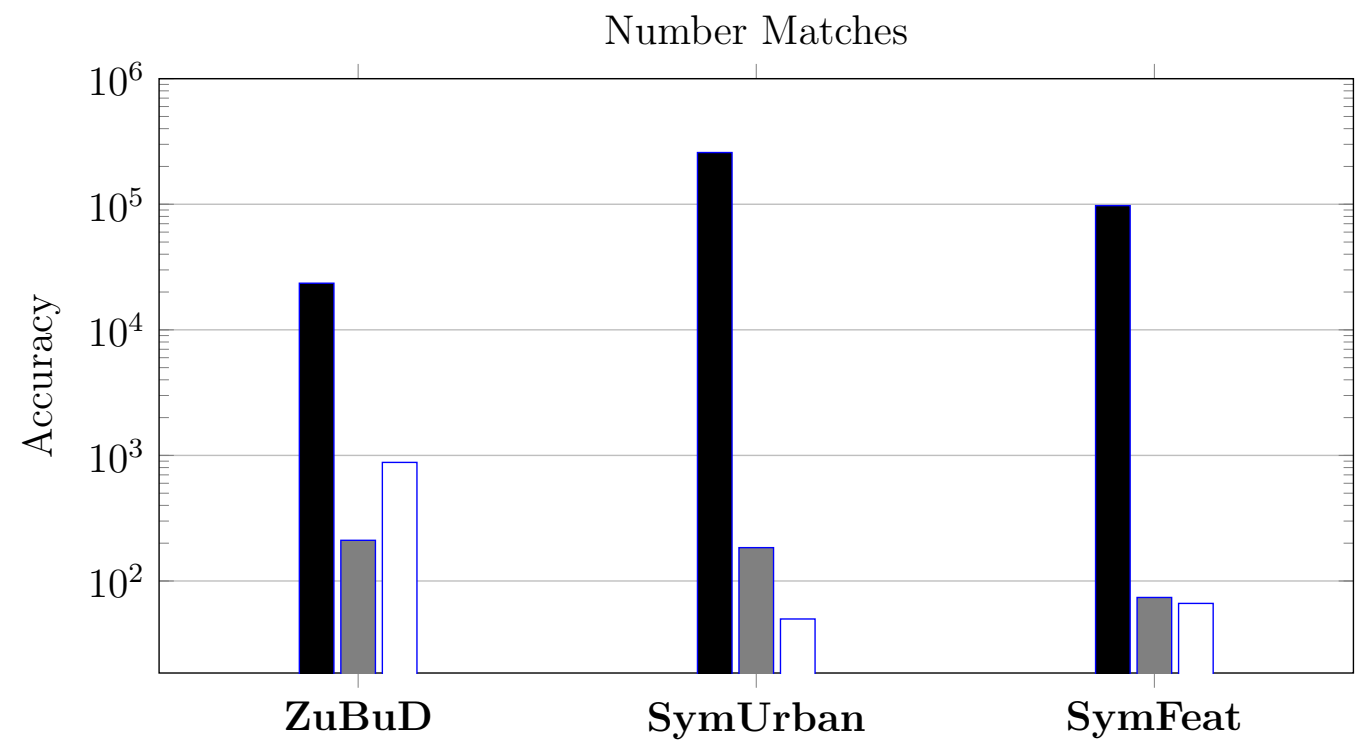

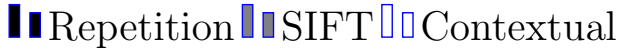

Figure 3.9: The number of matches found with the keypoint matching methods tested and shown in Figures 3.8 and 3.7. 

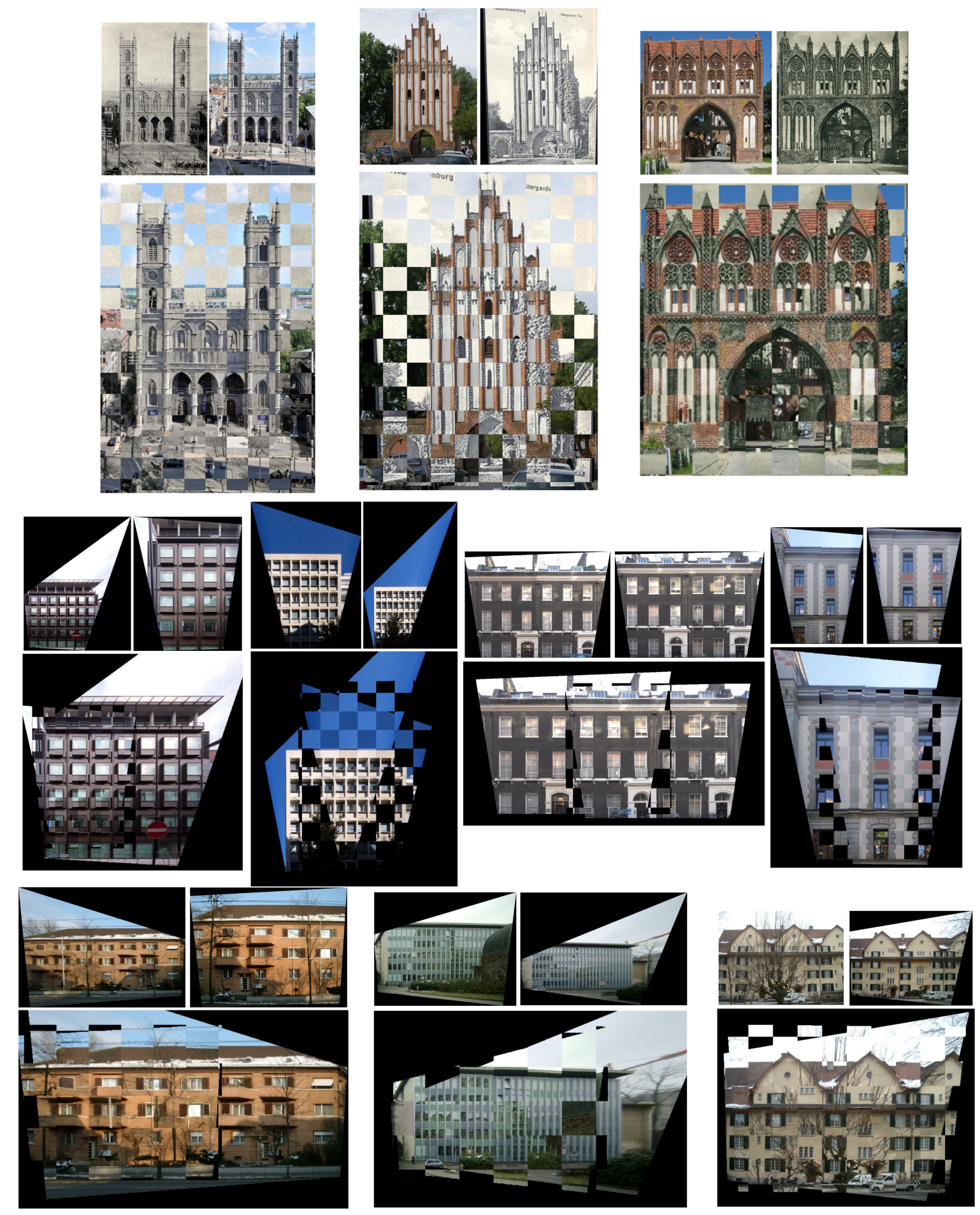

Figure 3.10: Visual alignment results. The original image pair is shown above each of the checkerboard views of the aligned result. These image pairs poses a variety of challenges to matching in addition to being repetitive such as different rendering styles, time changes, partial facade overlap, scale changes, and lighting changes. Image pairs shown are from datasets provided by [2], [5], and [6]. 


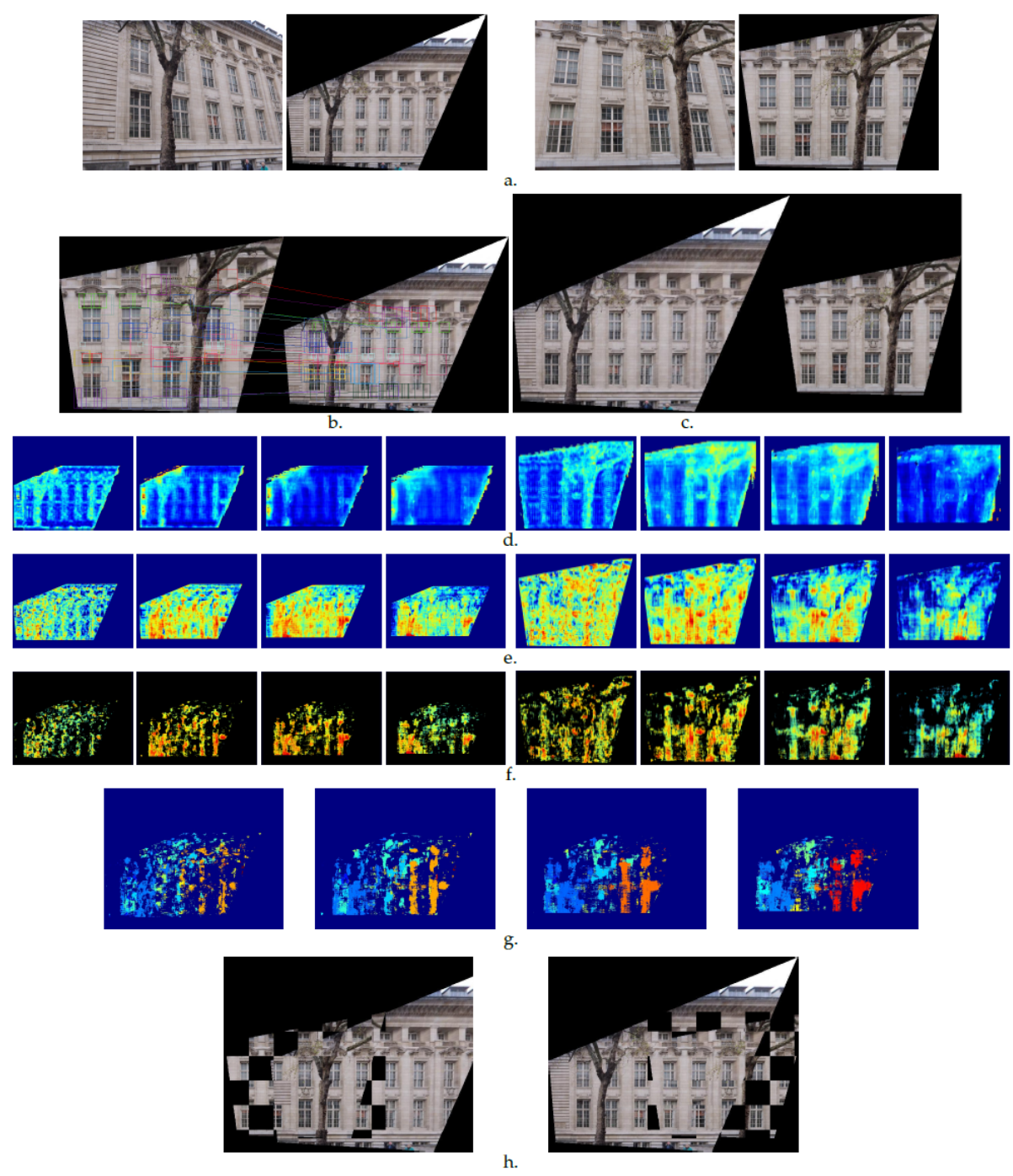

Figure 3.11: Alignment of images containing multiple planes. (a.) Original images, before and after rectification, that are being matched. (b.) Subset of the corresponding repetitive groups after computing a 1-D transformation (Section 3.3.1) are highlighted in matching colors. (c.) Images shown after they have been vertically aligned. (d.) Intra-image saliency map of each image. The four color fields for each image show the inner-image saliency computed at different scales. More salient areas appear in red and more repetitive areas appear in blue. (e.) Color fields of match saliency scores shown from the perspective of both images. (f.) Result after applying Otsu thresholding to match saliency score images. (g.) Displacement maps. Each match visualized in (f.) votes for an alignment. (h.) Result of aligning the image pair using the two different horizontal displacements chosen in (e.) The left result aligns the salient tree that is in front of (and on a different plane than) the building of interest. The right result uses the salient matches on the building facade to correctly align the region of interest. 


\section{Chapter 4}

\section{Image Super-Resolution with Deeply Supervised Nets and Incremental Learning}

\subsection{Introduction}

Single image super-resolution (SISR) refers to the task of reconstructing a HR image from a single low-resolution (LR) image. It is a well-known ill-posed problem due to the lack of additional information provided.

Recently, Kim et al. [60] proposed a residual convolutional neural network (VDSR) by concatenating small identical filters many times in a deep network structure to learn a nonlinear LR-to-HR mapping. This network outperforms SRCNN [61] in terms of accuracy and performance thanks to the use of many more layers and skipconnections. The accuracy of the network, however, starts to stall when the depth of the network is larger than 20. To overcome the difficulty of training when the depth is large, in this paper, we proposed SRDSN, a deeply supervised network for image super-resolution, which progressively predicts multiple intermediate HR images before being fused together as the final output. We show that fusing multiple intermediate 
HR images allows the network to learn better results in term of accuracy.

Our proposed SRDSN is related to HED [62] and DSN [63]. Since its inception, DSN has been applied to the task of boundary contour detection [62], but it has not been used for SISR.

The main contributions of this paper are: 1) we are the first group that applies that concept of DSN for SISR; 2) we proposed the idea of incremental training which can greatly reduce the training difficulty of deeper nets.

\subsection{Related Works}

The image super-resolution methods can be classified into two prominent categories: non-deep learning and deep learning methods. According to [64], the non-deep learning methods can be further categorized into four groups: prediction methods, edge based methods, image statistical methods and example-based methods. Most of the example-based and deep learning methods are currently widely used to model the mapping from LR to HR image patches.

\subsubsection{Non-deep learning methods}

Prediction methods: The algorithms in this group generate HR images from LR images through mathematical prediction models. Interpolation-based methods (bilinear, bicubic), inferring HR images by weighted averaging neighboring LR pixel values, tend to generate overly smooth images with artifacts along edges and high-frequency regions [65]. Irani et al. [66] proposed a method to interactively generate a LR image through a predefined downsampling model and compensated the difference map in LR back to the HR image. The contrast along edges is recovered better than the results generated by other interpolation algorithms.

Edge-Based methods: The ultimate goal of SISR is to preserve edges and 
high-frequency regions. Several SISR algorithms have been proposed to learn priors from edge features for reconstructing HR images. Dai et al. [67] modeled the local patches using the background and foreground descriptors and reconstructed the sharp discontinuity between them. Sun et al. [68] explored the gradient profile prior for local image structures and applied it to super-resolution. Since the priors are primarily learned from edges, the reconstructed HR images have high-quality edges with proper sharpness and limited artifacts. However, edge priors are less effective for modeling other high-frequency structures such as textures.

Image Statistical methods: Unlike the edge-based approaches, statistical algorithms relate the super-resolution (SR) reconstruction steps stochastically toward optimal reconstruction. Various algorithms have been proposed to exploit image properties as prior to predict HR images from LR images. Shan et al. [69] utilized the heavy-tailed gradient distribution [70] for SISR. Kim et al. [71] exploited the sparsity property of large gradients to reduce the computational load whereas Zhang et al. [72] used total variation as a regularization term for generating HR images.

Example-based methods: The non-linearity mapping which defines the relationship between the LR and HR images could be learned from a representative set of low and high-resolution image pairs, and then the mapping model is applied to a new LR image to obtain the most likely HR output [61,73-75]. These methods either exploit internal similarities of the same image [76-79] or learn mapping functions from external training image pairs $[71,73,75,80-85]$. Glasner et al. [78] proposed a method to combine example-based SR constraints and classical SR constraints in a unified framework which allows for inter-patch search, the parent of searched result is copied to an appropriate location in the high-resolution image. Freedman et al. [77] proposed a method to adopts a local search by using the multi-step coarse-to-fine algorithm. Since the extracted patches from multiple scale image may not always be sufficiently expressive to cover the textural appearance variations, Huang et al. [79] 
extended the self-similarity based SR method by allowing geometric variations in patch searching scheme. The sparse-coding-based methods $[75,80]$ are ones of the representative external example-based SR methods. In [75], Yang et al. proposed a super-resolution algorithm that learns a pair of over-complete dictionaries with the assumption that both the input and output patches have the same mixing coefficients of their corresponding dictionary.

\subsubsection{Deep-learning methods}

Recently, convolutional neural networks (CNNs) have demonstrated superior performance in the image SR thanks to their ability to exploit the contextual information through their receptive field to recover missing high-frequency components. Inspired from the traditional sparse coding-based SR [75], Dong et al. [86] proposed a shallow SRCNN consisting of three layers which correspond to patch representation, nonlinear mapping, and reconstruction. Kim et al. [60] proposed a residual network structure with 20 layers (VDSR) that significantly improves over SRCNN. Wang et al. [87] trained a cascade network (SCN) to upsample images to the desired scale factor progressively based on the knowledge of sparse coding. Kim et al. [88] proposed a deeply recursive convolutional neural network (DRCN) that utilizes a very large image context compared to previous SR methods with a single recursive layer.

To achieve real-time performance, Shi et al. [89] presented the first CNN (ESPCN) where feature map extraction is performed in LR space, and an efficient sub-pixel convolution is used to upscale the final LR feature maps to the HR image. Improving over the SRCNN, Dong et al. [90] introduced a fast version of SRCNN, named FSRCNN, which could reach up to 43 frames per second(fps) with a generic GPU. The drawback of this approach is that the upscale parameter needs to be determined in advance and different networks need to be trained for different scale factors. If we want to extend an existing network to a new upscale parameter, it needs to be re-trained $[89,90]$. 


\subsubsection{Deep-learning methods}

Recently, convolutional neural networks (CNNs) have demonstrated superior performance in the image SR thanks to their ability to exploit the contextual information through their receptive field to recover missing high-frequency components. Inspired from the traditional sparse coding-based SR [75], Dong et al. [86] proposed a shallow SRCNN consisting of three layers which correspond to patch representation, nonlinear mapping, and reconstruction. Kim et al. [60] proposed a residual network structure with 20 layers (VDSR) that significantly improves over SRCNN. Wang et al. [87] trained a cascade network (SCN) to upsample images to the desired scale factor progressively based on the knowledge of sparse coding. Kim et al. [88] proposed a deeply recursive convolutional neural network (DRCN) that utilizes a very large image context compared to previous SR methods with a single recursive layer.

To achieve real-time performance, Shi et al. [89] presented the first CNN (ESPCN) where feature map extraction is performed in LR space, and an efficient sub-pixel convolution is used to upscale the final LR feature maps to the HR image. Improving over the SRCNN, Dong et al. [90] introduced a fast version of SRCNN, named FSRCNN, which could reach up to 43 frames per second(fps) with a generic GPU. The drawback of this approach is that the upscale parameter needs to be determined in advance and different networks need to be trained for different scale factors. If we want to extend an existing network to a new upscale parameter, it needs to be re-trained $[89,90]$. 


\subsection{Deeply Supervised Nets with Incremental Learn- ing for Image Super-Resolution}

\subsubsection{Mathematical formulation}

Given an input of low-resolution image $x$, in SISR, the ultimate goal is to estimate a high-resolution image $\hat{y}=f(x)$ where $f$ is a predictor function. In this paper, we consider multiple predictors $\left(f_{1}, f_{2}, \ldots, f_{M}\right)$ which progressively produce $M$ intermediate outputs $\hat{y}_{m}, m=1,2, \ldots, M$, from input LR image $x$.

$$
\begin{aligned}
\hat{y}_{1} & =f_{1}(x) \\
\hat{y}_{m} & =f_{m}\left(\hat{y}_{m-1}\right)=f_{m}\left(f_{m-1}(\ldots(x))\right), \quad \text { for } \quad m=2 \rightarrow M
\end{aligned}
$$

The final HR image is averaged over all the intermediate outputs $\hat{y}=\sum_{i=1}^{M} \alpha_{i} \hat{y}_{m}$ with $\alpha_{i}$ indicates the relative importance of the intermediate output, e.g. setting $\alpha_{m}=0,1 \leq m<M$ makes the model turn into a predictor with only a single output at the top. For simplicity and consistency, we denote $g(x)=\sum_{i=1}^{M} \alpha_{i} \hat{y}_{m}$ as the final HR output.

In the context of deeply supervised nets (DSN), we consider the objective function that performs deep supervision for guiding to obtain an early high-resolution result.

$$
\mathcal{L}_{\text {loss }}=\sum_{m=1}^{M} \alpha_{m} l^{m}\left(\hat{y}_{m}, y\right)
$$

where $y$ is the ground truth image and $l_{m}$ is the mean square error (MSE) of the $m^{\text {th }}$ intermediate output $\hat{y}_{m}$, a common loss function for super-resolution problem. 

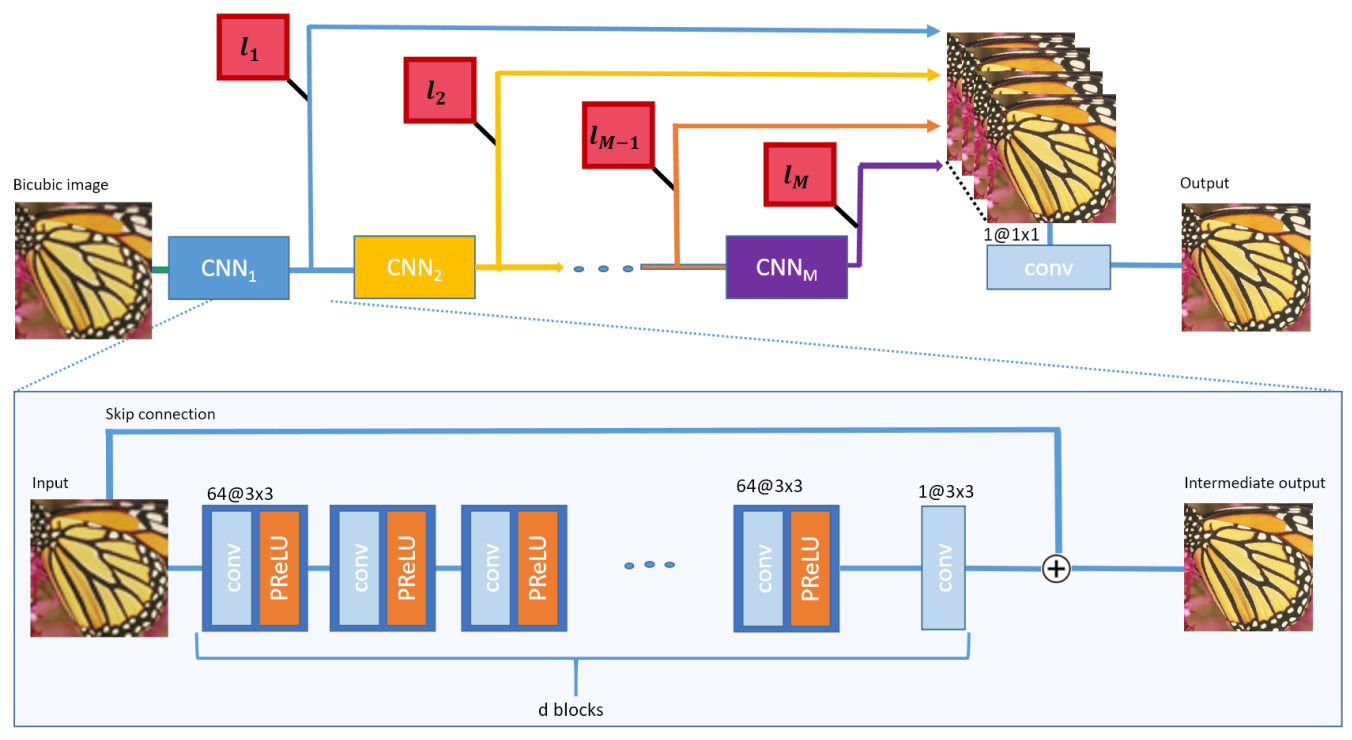

Figure 4.1: Our proposed network architecture: (Top) Multiple network blocks with deep supervision, every intermediate result is penalized by a loss function $l$. (Bottom) The layout of each network block with a corresponding number of feature maps followed by kernel size indicated for each convolutional (e.g. 64@3x3 stands for 64 $3 x 3$ kernels). Our final model consists of four blocks $(M=4)$ and 20 layers $(d=20)$ within each block.

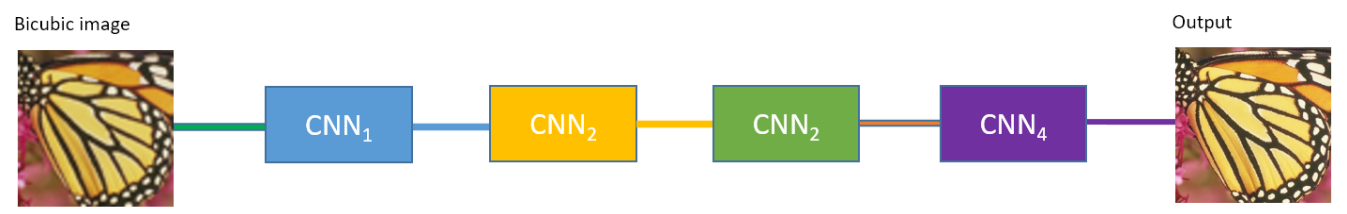

Figure 4.2: A simple stacked model with four network blocks. 


\subsubsection{Network architecture}

Our network structure is illustrated in Fig. 4.1. Each block represents an intermediate predictor function as described in the previous section. The network takes an upscaled version (e.g. bicubic) of the LR image as input and progressively predicts intermediate HR images. We supervise all the intermediate results to alleviate the effect of vanishing/exploding gradients. Our network contains two parts: (i) cascade network and (ii) deeply supervised network.

Cascade network: The network has multiple similar structure blocks. Each block, consisting of $d$ convolutional layers followed by PReLU except for the last one, takes an image ( 1 or 3 channels) to produce an intermediate HR image. This intermediate result is the input to the next block and is supervised by deeply supervised network.

Deeply supervised network: We supervise all the outputs of the network blocks using a DSN structure. The principle behind DSN can be considered as a network regularization that informs intermediate layers about the final objective, rather than relying on the final layer to back-propagate the information to its predecessors. The similar idea of supervising intermediate layers for a convolutional neural network can be found in HED [62] and DRCN [88].

Network properties: The proposed network looks similar to a recursive neural network with non-shared weights. For that reason, it has more power to model the non-linear mapping between LR and HR images since the number of parameters is more than that of a single block. However, it also tends to be exposed to over-fitting and is very hard to train due to the explosion of the parameters. In this paper, we propose a novel incremental learning technique which can greatly alleviate this issue. 


\subsubsection{Incremental Learning with DSN}

We denote our input training data set by $S=\left\{\left(x^{(n)}, y^{(n)}\right\}_{n=1}^{N}\right.$, where $x^{(n)}$ is the input LR image and $y^{(n)}$, only available during training, is the corresponding high-resolution ground truth image. Our goal is to estimate the learning function $f=\left(f_{1}, \ldots, f_{M}, g\right)$ that maps an input image $x$ in LR space to outputs in HR space $f(x)=\left(\hat{y}_{1}, \ldots, \hat{y}_{M}, \hat{y}\right)$. In the context of DSN, $\hat{y}_{n}$ is the intermediate result playing a role as a guidance for convergence and $\hat{y}$ is the estimation of the ground truth $y$.

Each intermediate output $\hat{y}_{m}$ is associated with a predictor $f_{m}$, in which the corresponding weights are denoted as $W=\left(W_{1}, \ldots, W_{m}\right)$. The intermediate losses are determined as mean square errors of the intermediate results and ground truth:

$$
l^{m}\left(\hat{Y}_{m}, Y, W_{m}\right)=\frac{1}{N} \sum_{i=1}^{N}\left\|{\hat{y_{m}}}^{(i)}-y^{(i)}\right\|^{2}
$$

We feed all the training data through the network to minimize the following objective function

$$
\mathcal{L}_{\text {loss }}(\hat{Y}, Y, W)=\sum_{m=1}^{M} \alpha_{m} l^{m}\left(\hat{Y}_{m}, Y, W_{m}\right)
$$

It is worth mentioning that the relative importance factors $\alpha_{m}, m=1, \ldots, M$ can also be learned during training.

Incremental Learning: Obviously, the network has higher complexity when the number of network blocks increases. For example, the parameter number of the 2block network is approximately two times larger than that of the 1-block network. Although multi-block network has more power to model the LR-HR relationship, training such a deep network is not trivial due to the larger complexity. Our idea of using incremental learning for training is very simple: (1) start by training one block first which is relatively easier to train, (2) incrementally add addition blocks and fine 
train the network for each training stage.

It is worth noting that the objective function needs to be updated when a network block is added. To update the objective function during the training process, we modify the objective function at equation 4.4 by introducing auxiliary variables $\Pi=$ $\left(\pi_{1}, \ldots, \pi_{M}\right)$ which take binary values $\{0,1\}$. Each $\pi_{i}$ is either on or off to adapt to each training stage.

$$
\mathcal{L}_{\text {total }}(\hat{Y}, Y, W)=\sum_{m=1}^{M} \frac{\pi_{m}}{\# n} \alpha_{m} l^{m}\left(\hat{Y}_{m}, Y, W_{m}\right)
$$

where $\# n$ is number of non-zero elements of $\Pi$.

For simplicity, we use the term "model capacity" to infer to the number of the model parameters. Let denote $n_{1}, \ldots, n_{M}$ as the number of parameters from the first block to the $M^{\text {th }}$ one, respectively. At the first stage of training, we only train the first block of the network by keeping all the auxiliary variables are 0's except the first one. The model capacity is only $n_{1}$ at this stage. At the second stage of training, we set $\pi_{2}=1$ and initialize $W_{2}=W_{1}^{(1)}$ and the model capacity is $n_{1}+n_{2}+2$ and so on. At the final stage of training, all the auxiliary variables are 1's; the objective function becomes our defined loss function as described in (4.4). Although the model capacity at the final stage is still the same with the original objective function, the proposed network does not suffer from common training difficulty of deep nets thanks to good initialization obtained from the previous stage. Table 4.3 provides more detail of incremental learning for the case of $M=4$.

\subsection{Experiments}

In this section, we evaluate the performance of our method on several datasets. We first describe datasets used for training and testing our method. Next, parameters 
Table 4.1: Incremental Learning with $M=4$

\begin{tabular}{|r|r|r|r|r|}
\hline Stage & Objective function & Model capacity & Weight initialization & Solutions \\
\hline \hline 1 & $\alpha_{1} l^{m}\left(\hat{y}_{1}, y\right)$ & $n_{1}$ & $W_{1}=W_{1}^{(0)}$ as described in $[90]$ & $\left(W_{1}^{(1)}, 1.0\right)$ \\
\hline 2 & $\sum_{m=1}^{2} \frac{\alpha_{i}}{2} l^{m}\left(\hat{y}_{m}, y\right)$ & $\sum_{m=1}^{2} n_{m}+2$ & $\left(W_{m}, \alpha_{m}\right)= \begin{cases}\left(W_{m}^{(1)}, 1\right), & m=1 \\
\left(W_{1}^{(1)}, 1\right), & m=2\end{cases}$ & $\left\{\left(W_{m}^{(2)}, \alpha_{m}^{(2)}\right), m=\overline{1,2}\right\}$ \\
\hline 3 & $\sum_{m=1}^{3} \frac{\alpha_{i}}{3} l^{m}\left(\hat{y}_{m}, y\right)$ & $\sum_{m=1}^{3} n_{m}+3$ & $\left(W_{m}, \alpha_{m}\right)= \begin{cases}\left(W_{m}^{(2)}, \alpha_{m}^{(2)}\right), & m \leq 2 \\
\left(W_{2}^{(3)}, 1\right), & m=3\end{cases}$ & $\left\{\left(W_{m}^{(3)}, \alpha_{m}^{(3)}\right), m=\overline{1,3}\right\}$ \\
\hline 4 & $\sum_{m=1}^{4} \frac{\alpha_{i}}{4} l^{m}\left(\hat{y}_{m}, y\right)$ & $\sum_{m=1}^{4} n_{m}+4$ & $\left(W_{m}, \alpha_{m}\right)= \begin{cases}\left(W_{m}^{(3)}, \alpha_{m}^{(3)}\right), & m \leq 3 \\
\left(W_{3}^{(3)}, 1\right), & m=4\end{cases}$ & $\left\{\left(W_{m}^{(4)}, \alpha_{m}^{(4)}\right), m=\overline{1,4}\right\}$ \\
\hline
\end{tabular}

necessary for training are given. After outlining our experimental setup, we compare our method with several state-of-the-art SISR methods.

\subsubsection{Datasets}

For training, following [60], we train our model on 291 images, where 91 images from Yang et al. [75] and other 200 images from Berkeley Segmentation Dataset [91]. For testing, we use some standard benchmark datasets including Set5 [92], Set14 [93], BSD100 [93] and Urban100 [79], which consists of 5, 14, 100 and 100 images respectively.

\subsubsection{Implementation details}

Data samples: To prepare the training data, we first downsample the original training images by the desired scaling factor to obtain the LR images, then we upscale the LR images to desired size images that have the same sizes as the HR images. Both of the downsample and upsample methods are based on bicubic interpolation. We sample 400K patches from the upscaled LR images with a stride of 20 . The corresponding HR patches are also cropped from the ground truth images. These LR and HR patch pairs are the primary training data.

Data augmentation: Inspired by [94], we augment the training data in two ways: (i) Rotation: rotate images by $90^{\circ}, 180^{\circ}$ and $270^{\circ}$; (ii) Flipping: flip images 
Table 4.2: Benchmark results. Average PSNR/SSIMs for scale factor $\times 2, \times 3$ and $\times 4$ on datasets Set5, Set14, B100 and Urban100. Red color indicates the best performance and blue color refers the second best

\begin{tabular}{|c|c|c|c|c|c|c|c|c|c|}
\hline Dataset & Scale & $\begin{array}{c}\text { Bicubic } \\
\text { PSNR/SSIM }\end{array}$ & $\begin{array}{c}\mathrm{A}+[84] \\
\mathrm{PSNR} / \mathrm{SSIM}\end{array}$ & $\begin{array}{c}\text { RFL [82] } \\
\text { PSNR/SSIM }\end{array}$ & $\begin{array}{c}\text { SelfEx [79] } \\
\text { PSNR/SSIM }\end{array}$ & $\begin{array}{l}\text { SRCNN [86] } \\
\text { PSNR/SSIM }\end{array}$ & $\begin{array}{c}\text { VDSR [60] } \\
\text { PSNR/SSIM }\end{array}$ & $\begin{array}{c}\text { DRCN [88] } \\
\text { PSNR/SSIM }\end{array}$ & $\begin{array}{l}\text { SRDSN(ours) } \\
\text { PSNR/SSIM }\end{array}$ \\
\hline \multirow{3}{*}{ Set5 } & & $33.66 / 0.9299$ & $36.54 / 0.9544$ & $36.54 / 0.9537$ & $36.49 / 0.9537$ & $36.66 / 0.9542$ & $37.53 / 0.9587$ & $37.63 / 0.9588$ & $37.67 / 0.9593$ \\
\hline & & $0.39 / 0.8682$ & $32.58 / 0.9088$ & $32.43 / 0.9057$ & $32.58 / 0.9093$ & $32.75 / 0.9090$ & $33.66 / 0.9213$ & $33.82 / 0.9226$ & $33.91 / 0.9231$ \\
\hline & & $28.42 / 0.8104$ & $30.28 / 0.8603$ & $30.14 / 0.8548$ & $30.31 / 0.8619$ & $30.48 / 0.8628$ & $31.35 / 0.8838$ & $31.53 / 0.8854$ & $31.68 / 0.8887$ \\
\hline \multirow{3}{*}{ Set14 } & $\overline{\times 1 \times 2}$ & $30.24 / 0.8688$ & $32.28 / 0.9056$ & $32.26 / 0.9040$ & $32.22 / 0.9034$ & $32.42 / 0.9063$ & $33.03 / 0.9124$ & $33.04 / 0.9118$ & $33.26 / 0.9142$ \\
\hline & & $27.55 / 0.7742$ & $29.13 / 0.8188$ & $29.05 / 0.8164$ & $29.16 / 0.8196$ & $29.28 / 0.8209$ & $29.77 / 0.8314$ & $29.76 / 0.8311$ & $30.05 / 0.8350$ \\
\hline & $\times 4$ & $26.00 / 0.7027$ & $27.32 / 0.7491$ & $27.24 / 0.7451$ & $27.40 / 0.7518$ & $27.49 / 0.7503$ & $28.01 / 0.7674$ & $28.02 / 0.7670$ & $28.38 / 0.7750$ \\
\hline \multirow{3}{*}{ B100 } & $\overline{\mathrm{x} 2}$ & $29.56 / 0.8431$ & $31.21 / 0$. & $\overline{31.16 / 0.8}$ & $31.18 / 0.8855$ & $31.36 / 0$ & (31.90/0.8960 & $31.85 / 0.8942$ & 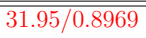 \\
\hline & & $27.21 / 0.7385$ & $28.29 / 0.7835$ & $28.22 / 0.7806$ & $28.29 / 0.7$ & $28.41 / 0.7863$ & $28.82 / 0.7976$ & $28.80 / 0.7963$ & $28.90 / 0.7989$ \\
\hline & $\times 4$ & $25.96 / 0.6675$ & $26.82 / 0.7087$ & $26.75 / 0.7054$ & $26.84 / 0.7106$ & $26.90 / 0.7101$ & $27.29 / 0.7251$ & $27.23 / 0.7233$ & $27.40 / 0.7286$ \\
\hline \multirow{3}{*}{ Urban100 } & $\bar{x}$ & $26.88 / 0.8403$ & $29.20 / 0.8938$ & $29.11 / 0.8904$ & $29.54 / 0.8967$ & $29.50 / 0.8946$ & $30.76 / 0.9140$ & $30.75 / 0.9133$ & $31.07 / 0.9173$ \\
\hline & & $24.46 / 0.7349$ & $26.03 / 0.7973$ & $25.86 / 0.7900$ & $26.44 / 0.8088$ & $26.24 / 0.7989$ & $27.14 / 0.8279$ & $27.15 / 0.8276$ & $27.38 / 0.8329$ \\
\hline & & $23.14 / 0.6577$ & $24.32 / 0.7183$ & $24.19 / 0.7096$ & $24.79 / 0.7374$ & $24.52 / 0.7221$ & $25.18 / 0.7524$ & $25.14 / 0.7510$ & $25.54 / 0.7641$ \\
\hline
\end{tabular}

horizontally or vertically. These together lead to an augmented training set that is a factor of 8 times larger than the original data set.

Training details: We implement our network using public deep learning framework Lasagne/Theano [95,96]. For simplicity, we represent our proposed SRDSN network as $\operatorname{SRDSN}(d, M)$ where $d$ is a number of convolutional layers in a block and $M$ is a number of the blocks in the model. Empirically, we choose the following hyper-parameters: minibatch size (32), convolutional filters and bias randomly initialized as described in [90]. We adopt the adjustable gradient clipping [60] to boost the convergence rate while suppressing exploding gradients. Specifically, the gradients are clipped to $\left[-\frac{\theta}{\gamma} ; \frac{\theta}{\gamma}\right]$, where $\gamma$ is the current learning rate and $\theta=0.01$ is the gradient clipping parameter. Furthermore, we use Adam optimizer [97] with initial learning rate $10^{-4}$. We train the network in 40 epochs with four training stages. Each training stage is performed in 10 epochs. Training takes four days on a machine using a Nvidia TitanX GPU.

\subsubsection{Investigation of Different Settings}

To test the property of the SRDSN structure, we design a set of controlled experiments with different values of the two sensitive parameters the number of the convolutional 
layer within a block $d$ and the number of blocks $M$. Specifically, we choose $d=$ $10,15,20,25$ and $m=1,2,3,4$, thus we conduct a total of 16 experiments with different combinations. The trend of the average PSNR $(\times 4)$ values on the Set14 dataset of these experiments are shown in Fig. 4.3a and Fig. 4.3b. First, we fix $d$ and examine the influence of $M$. Obviously, $M=4$ leads to better results than $M=2$ and $M=3$. Second, we fix $M$ and examine the influence of $d$. We also observe the same phenomenon when $d$ is increased. However, when $d$ is larger than 20, the network converges (Fig. 4.3b). From all the observed results, we find the best network configuration $\operatorname{SRDSN}(20,4)$, which achieves the highest accuracy.

We also evaluate the effect of deeply supervised nets and incremental learning strategy. We perform this evaluation on the networks which have the same blocks $(M=4)$ and same convolution layers within a block $(d=20)$. The first one is just a simple stacked model as shown in Fig. 4.2, the second one is the proposed network without incremental learning, and the final one is the full model SRDSN(20.4). As we can see in Fig. 4.3d, while both SRDSN without incremental learning and stacked model converge very fast and get flat after $100 \mathrm{~K}$ iterations, the complete model SRDSN improves significantly whenever the training process is moved to the next stage. More specifically, both $\operatorname{SRDSN}(20,4)$ without incremental learning and stacked model obtain PSNR of 28.04 and 28.06 respectively whereas the $\operatorname{SRDSN}(20,4)$ achieve PSNR of 28.37 .

\subsubsection{Single Model for Multiple Scales}

Similar to [60], we also evaluate the accuracy of the network with scale augmentation which is capable of performing SR at multiple scale factors. For example, to train the network with scale augmentation $s_{\text {train }}=(\times 2, \times 3, \times 4)$, we sequentially downsample an original image to scale factors of 2, 3, 4 to obtain three LR images, then we use bicubic interpolation to upscale those $\mathrm{LR}$ images back to the original image size. We 


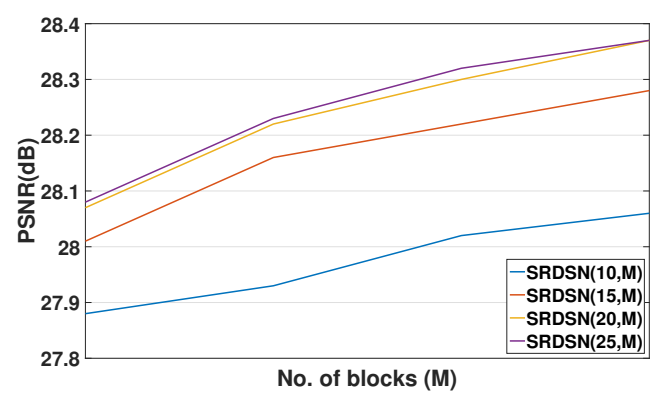

(a)

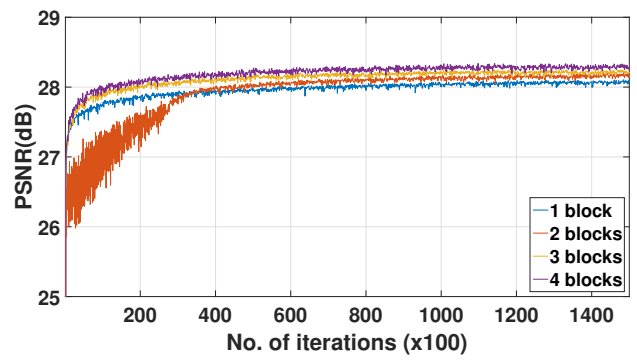

(c)

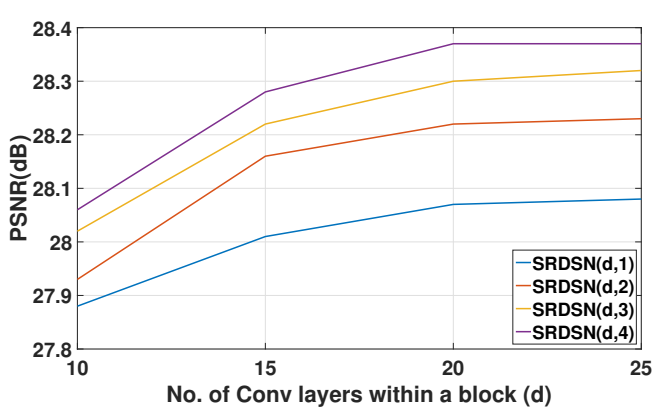

(b)

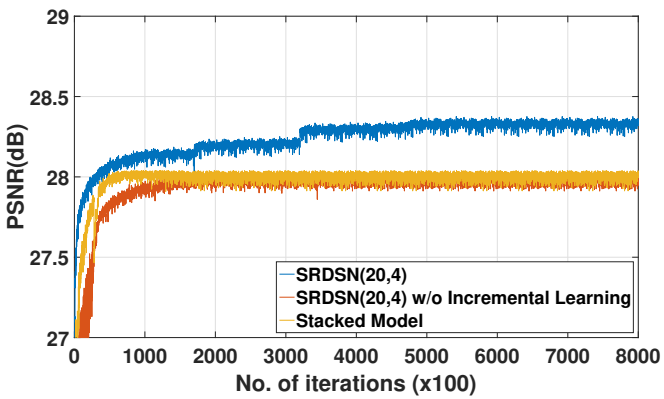

(d)

Figure 4.3: Evaluations performed on Set14 dataset. (a): Number of blocks versus accuracy for the scale factor $\times 4$. More blocks mean deeper network which leads to better accuracy. (b): Number of block's layers versus accuracy for the scale factor $\times 4$. More layers within a block yield deeper network which leads to better accuracy. (c): Learning curve of various SRDSN at different configurations. We fix the number of the convolutional layer for each block and change the number of blocks. More blocks yield better accuracy. (d) Learning curve of SRDSN, SRDSN w/o incremental learning, and stacked model. Both SRDSN w/o incremental learning and stacked model flat out very quickly while SRDSN improves significantly after stage training transitions. 
Table 4.3: PSNR on Set14(×4) with different network configurations

\begin{tabular}{|r|r|r|r|r|}
\hline No. & Network & No. of blocks & No. of layers & PSNR \\
\hline 1 & $\operatorname{SRDSN}(10,1)$ & 1 & 10 & 27.88 \\
2 & $\operatorname{SRDSN}(15,1)$ & 1 & 15 & 28.01 \\
3 & $\operatorname{SRDSN}(20,1)$ & 1 & 20 & 28.07 \\
4 & $\operatorname{SRDSN}(25,1)$ & 1 & 25 & 28.08 \\
\hline 5 & $\operatorname{SRDSN}(10,2)$ & 2 & 10 & 27.93 \\
6 & $\operatorname{SRDSN}(15,2)$ & 2 & 15 & 28.16 \\
7 & $\operatorname{SRDSN}(20,2)$ & 2 & 20 & 28.22 \\
8 & $\operatorname{SRDSN}(25,2)$ & 2 & 25 & 28.23 \\
\hline 9 & $\operatorname{SRDSN}(10,3)$ & 3 & 10 & 28.02 \\
10 & $\operatorname{SRDSN}(15,3)$ & 3 & 15 & 28.22 \\
11 & $\operatorname{SRDSN}(20,3)$ & 3 & 20 & 28.30 \\
12 & $\operatorname{SRDSN}(25,3)$ & 3 & 25 & 28.32 \\
\hline 13 & $\operatorname{SRDSN}(10,4)$ & 4 & 10 & 28.06 \\
14 & $\operatorname{SRDSN}(15,4)$ & 4 & 15 & 28.28 \\
15 & $\operatorname{SRDSN}(20,4)$ & 4 & 20 & 28.37 \\
16 & $\operatorname{SRDSN}(25,4)$ & 4 & 25 & 28.37 \\
\hline
\end{tabular}

Table 4.4: PSNR on Set14 $(\times 4)$ with scale augmentations. Red color indicates the best performance and blue color refers the second best.

\begin{tabular}{|r|r|r|r|r|r|r|r|}
\hline Test/Train & $\times 2$ & $\times 3$ & $\times 4$ & $\times 2,3$ & $\times 2,4$ & $\times 3,4$ & $\times 2,3,4$ \\
\hline$\times 2$ & 33.26 & 30.20 & 27.63 & 33.24 & 33.24 & 28.01 & 30.23 \\
$\times 3$ & 27.01 & 30.05 & 27.63 & 30.03 & 27.86 & 30.04 & 30.02 \\
$\times 4$ & 24.22 & 25.29 & 28.38 & 24.56 & 28.30 & 28.28 & 28.32 \\
\hline
\end{tabular}

perform multiple scale combinations for scale augmentation. Experimental results are summarized in Table 4.4 with testing results evaluated on Set14 dataset. We observe that the network trained over single scale data is not capable of handling other scales. For scale factor 4 , the model trained with factor 4 gives PSNR of 28.38 (in $\mathrm{dB}$ ), while models trained with factor 2 and 3 give 25.43 and 22.32, respectively. In contrast, the network trained with multiple scale factors, $s_{\text {train }}=(\times 2, \times 3, \times 4)$, its PSNR for each scale is comparable to those obtained from the corresponding result of single scale network: 33.23 vs. $33.26(\times 2), 30.02$ vs. $30.05(\times 3), 28.32$ vs $28.38(\times 4)$. 


\subsubsection{Comparisons with State-of-the-Art Methods}

We provide quantitative and qualitative comparisons. For benchmark, we use public code for A+ [84], SRCNN [86], RFL [82] and [79], VDSR [60] and DRCN [88]. Similar to all other state-of-the-art algorithms, we only deal with an illumination component and crop pixels to the same amount for a fair comparison. In Table 4.2, we provide a summary of quantitative evaluation on several datasets. Our method outperforms all existing methods in all datasets and scale factors (both PSNR and SSIM). In Fig. 4.4, example images are given. Our method produces relatively sharp edges respective to patterns. In contrast, edges in other images are blurred. Our method takes a second to process a $256 \times 256$ image on a GPU Titan X.

\subsection{Conclusion}

In this work, we propose a single image super-resolution with deeply supervision nets and incremental learning. Our model progressively predicts intermediate highresolution images and combine them to obtain the final high-resolution result. By cascading multiple similar structure blocks and optimizing the network with an incremental learning strategy, our proposed SRDSN alleviates the common difficulty of training a very deep network. Quantitative evaluations on benchmark datasets demonstrate that the proposed model performs favorably against the state-of-the-art SR algorithms in terms of visual quality.

\subsection{Limitation and Future Work}

SRDSN uses bicubic interpolation image as its input, which has some trade-offs. The network can support multiple scale factors as shown in Section 4.4.4. However, it is not efficient due to the fact that working on HR space requires more memory and 


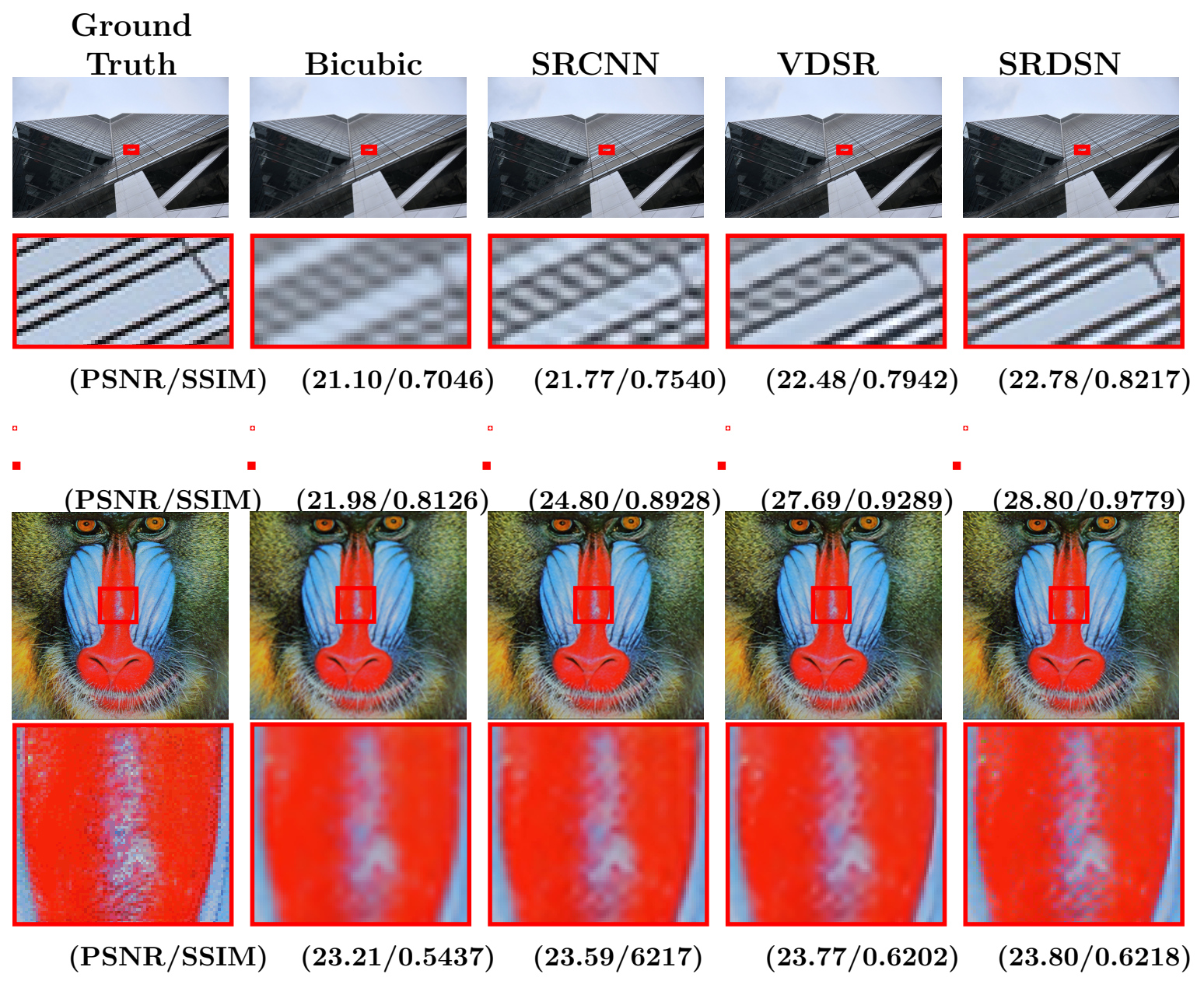

Figure 4.4: Comparisons with other methods $(\times 3)$. Each image row is followed by a zoom-in image row marked with red rectangles. (1) Super-resolution results of "img059" (Urban100) with scale factor x3. SRDSN recovers sharp lines. (2) The second example shows image "ppt3" (Set14 with scale factor x3). Texts in SRDSN are sharp, while others are blurry. (3) The third row shows image "baboon" (Set15 with scale factor $\mathrm{x} 3$ ). SRDSN recovers high frequency regions, while others all give blurry results. 


\section{Ground}
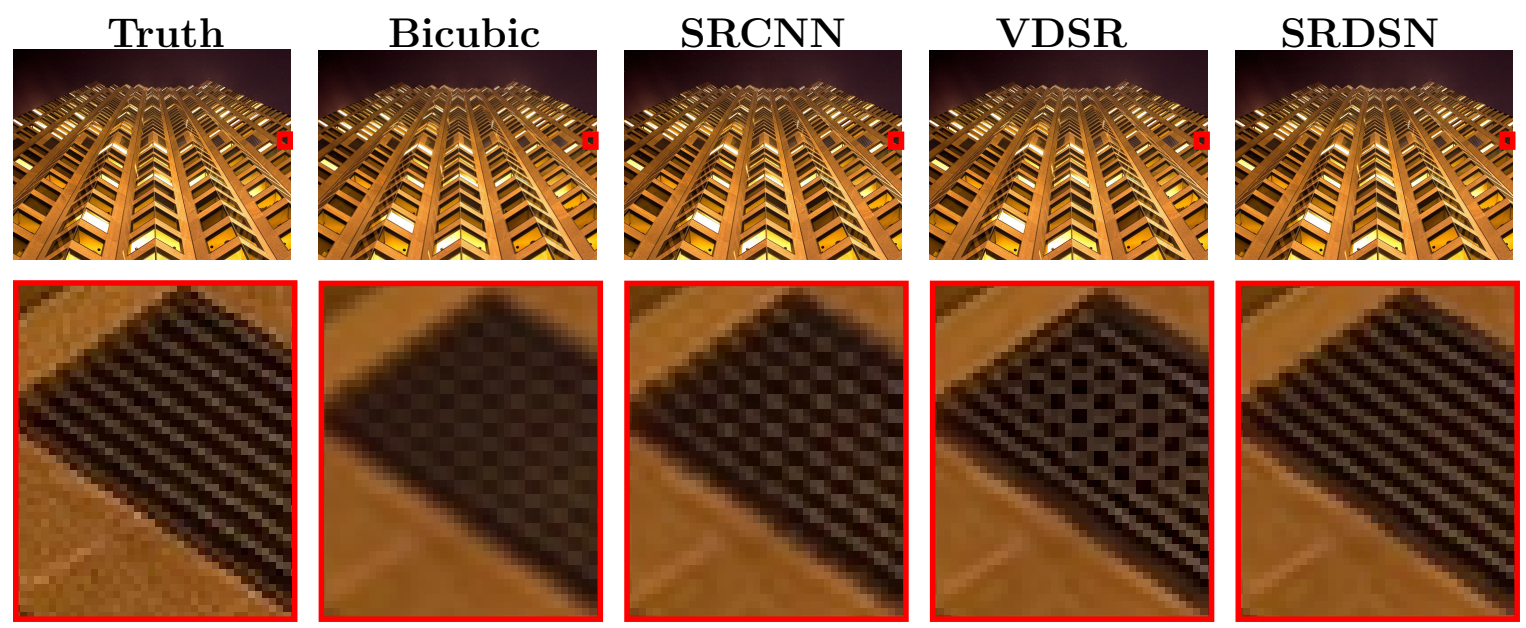

(PSNR/SSIM)

$(25.88 / 0.7635)$

$(28.52 / 0.8277)$

$(29.82 / 0.8473)$
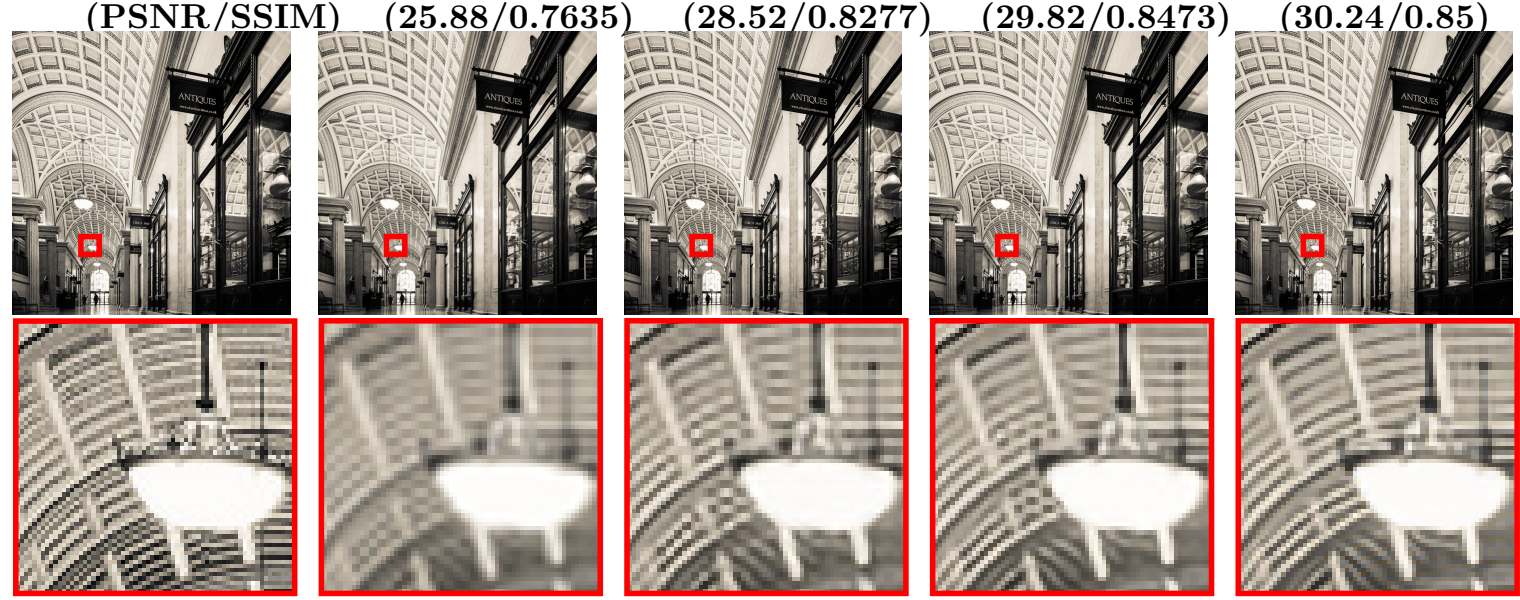

(PSNR/SSIM)

$(23.45 / 0.7809)$

$(25.24 / 0.8558)$

$(26.09 / 0.8849)$

$(26.25 / 0.890858)$

Figure 4.5: Comparisons with other methods $(\times 2)$. Each image row is followed by a zoom-in image row marked with red rectangles: (1) Super-resolution results of "img015" (Urban100) with scale factor x2. SRDSN recovers sharp lines. (2) The second example shows image "img083" (Urban100 with scale factor x2). SRDSN recovers the pattern. 


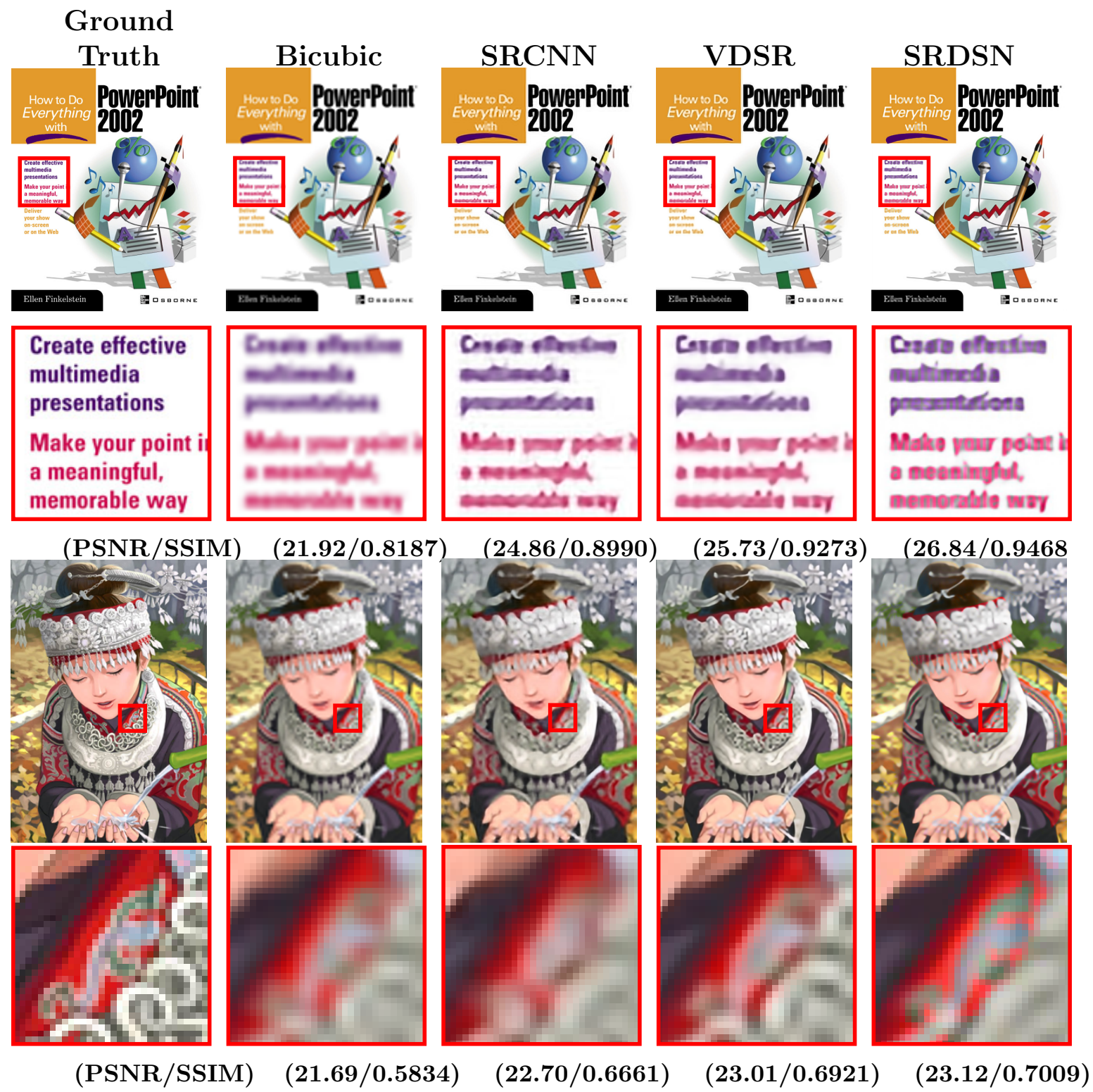

Figure 4.6: Comparisons with other methods $(\times 4)$. Each image row is followed by a zoom-in image row marked with red rectangles: (1) Super-resolution results of "ppt3" (Set14) with scale factor $\mathrm{x} 4$. SRDSN recovers sharper text. (2) The second example shows image "comic" (Set14 with scale factor x4) SRDSN recovers high-frequency regions, while others all give blurry results. 
computation. In the future work, we plan to investigate a network which works directly on a LR image instead of an interpolation image and uses upsampling operators in a later stage. 


\section{Chapter 5}

\section{Point Based Rendering Enhancement via Deep Learning}

\subsection{Overview}

Large scale 3D scene modeling and visualization are fundamental building blocks for active research fields such as Virtual Reality, Augmented Reality, Autonomous Driving, etc. Despite decades of researches in this area, how to model the real world efficiently and accurately remains a very challenging task.

The advancement of 3D depth sensors, such as LIDAR (Light Detection And Ranging) scanners, has provided an effective alternative to traditional CAD-based and image-based approaches for 3D modeling. The output of these 3D depth sensors is generally 3D point clouds with color information. However, current state-of-art point rendering techniques such as splat rendering generally require a very high resolution of point clouds in order to create a high quality photo realistic rendering which could be very time consuming to acquire and often also requires high end expensive scanners. We proposes a novel deep learning based approach that can generate high resolution photo realistic point renderings from low resolution point clouds. The pro- 
posed method is inspired by recent works on image super resolution (SR) via deep learning techniques.

\section{$5.2 \quad$ Related Works}

Point-based splat rendering is a technique for rendering a smooth surface with approximated linear piece-wise splats. It was originally proposed by Zwicker et al. [98]. In order to cover the gaps between the points, a circular disc is assigned to each sample point with a normal vector $n_{i}$ and radius $r_{i}$ which are computed based on local geometry. The surface splats serve as linear approximation to a smooth surface if constructed properly. In order to remove the sharp edges between splats, Botsch et al. introduced a multi-pass rendering approach which averages colors and normals of overlapping splats [99]. However, this approach may generate blurred images due to gradient suppression. Recently, Sibbing et al. [100] proposed two post-processing methods to further improve the results of splat rendering. Intensity Completion preserves the intensities of the non-gap pixels, and automatically finds color transitions between the non-gap and gap pixels while Gradient Completion requires additional gradient information from intermediate images to reconstruct image more faithfully. Moreover, when additional texture images are available along with projection matrices, they also proposed Texture Splat Rendering which improves the rendered image by propagating as much information as possible from a set of texture images. That is to say, instead of using a single color for a whole splat, they used a projection matrix to project the fragments of the splat into a texture image and obtain the color information. However, obtaining the projection matrices of texture images requires non-trivial 2D-3D registration, and storing all the textures images together with a large point cloud requires a lot of memory. Similar to Intensity Completion and Gradient Completion of [100], we also propose to conduct post-processing image 
enhancement based on the 3-pass splat rendering results of [99]. Compared with Intensity Completion and Gradient Completion methods, our results are much better. Compared with Texture Splat Rendering, our method does not need to store extra texture images while still generate better image quality. Fig. 5.1 shows a side-by-side comparisons between the proposed method and the photograph, 3-pass rendering [99], Intensity Completion, Gradient Completion, Texture Splat Rendering. More examples are shown in Fig. 5.4 and 5.5.

Image Super Resolution (SR) is a set of methods to reconstruct a high-resolution image from either a single or multiple images. The image super-resolution methods can be classified into 3 categories: interpolation-based method, reconstruction-based method, and example-based method. According to [64], example-based methods show superior performance by modeling the mapping from low-resolution (LR) to highresolution (HR) image patches, and then applying this map to a new LR image to obtain the most likely HR output [61,73-75]. These methods either exploit internal similarities of the same image [76-79] or learn mapping functions from external training image pairs [71,73,75,80-85]. Glasner et al. [78] proposed a method to combine example-based SR constraints and classical SR constraints in a unified framework which allows for inter-patch search. The parent of the search results is copied to an appropriate location in the high-resolution image. Freedman et al. [77] proposed a method to adopt a local search by using the multi-step coarse-to-fine algorithm. Since the extracted patches from multiple scale images may not always be sufficiently expressive to cover the textural appearance variations, Huang et al. [79] extended the self-similarity based SR method by allowing geometric variations in patch searching scheme. The sparse-coding-based methods $[75,80]$ are the representative external example-based SR methods. Yang et al. [75] proposed a super-resolution algorithm that learns a pair of over-complete dictionaries with the assumption that both the input and output patches have the same mixing coefficients of their corresponding 
dictionary.

Besides traditional super-resolution methods [61,73-75,77,78,80], convolutional neural networks (CNNs) have recently demonstrated remarkable performance in the image SR field thanks to their ability to exploit the contextual information through their receptive field to recover missing high frequency components. Inspired by traditional sparse coding based SR [75], Dong et al. [86] proposed a shallow SRCNN consisting of three layers which correspond to patch representation, non-linear mapping, and reconstruction. Kim et al. [88] proposed a deeply recursive CNN that utilizes a very large image context compared to previous SR methods with a single recursive layer. Kim et al. [60] proposed a residual network structure with 16 layers by concatenating $643 \times 3$ kernels. For these approaches, the SR methods work directly on the high-resolution (HR) space by first applying a simple interpolation method (e.g. bicubic interpolation) to upsample the LR image to the desired size, then feeding it through a deep neural network to obtain a visually satisfying result. While most of the deep learning based super image super resolution (SISR) methods work directly on the HR space, other methods cope with the LR space and only go back to the HR space at the very last several layers. Bishop et al. [89] presented the first CNN in which extracted feature maps are performed in LR space and an efficient sub-pixel convolution is used to upscale the final LR feature maps to the HR image. Improving over the SRCNN, Dong et al. [90] introduced a fast version of SRCNN, named FSRCNN, which can reach up to 43 fps with a generic GPU. Ledig et al. [101] proposed SRResNet by employing the ResNet architecture from He at al. [102] that successfully solves time and memory issues with good performance. By removing the batch normalization layers from SRResNet, Lim et al. [103] showed superior performance over the state-of-the-art methods and won the NTIRE2017 Super-Resolution Challenge [104].

Recently, there has been a lot of interest in Generative Adversarial Networks 89 


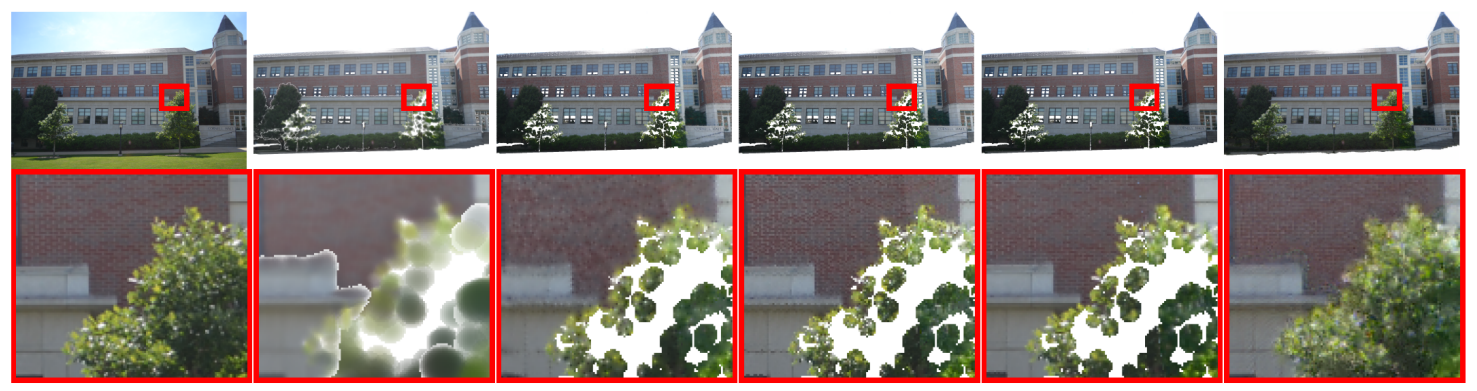

Figure 5.1: Different rendering techniques: (1) Photograph. (2) Splat rendering. (3) Intensity completion. (4) Gradient completion. (5) Texture splat rendering. (6) (Ours) Deep-Learning based rendering with splat rendering of (2) as input.

(GANs) [105] which can learn to produce samples that imitate the dataset according to the discriminator network. Mathieu et al. [106] supplemented a squared error loss with both GAN and image gradient-based similarity to improve image sharpness of video prediction. The ability to produce high-quality images of GANs also demonstrated in the works of Denton et al. [107] and Radford et al. [108]. In the work of Johnson et al. [109] and Ledig et al. [101], the authors proposed a conceptual loss function based on the VGG network [110] obtained the results that are more convincing than the ones obtained with traditional low level mean square error (MSE). In this work, we employ the GAN network for point based rendering enhancement, which will be explained in more details in the following section.

\subsection{Proposed Method}

Fig. 5.2 shows the overview of our proposed method. The algorithm takes a splat image as input and feeds it through a neural network to obtain a high quality image. As shown in Fig. 5.1, the deep learning result is almost indistinguishable from the photograph taken by cameras. 


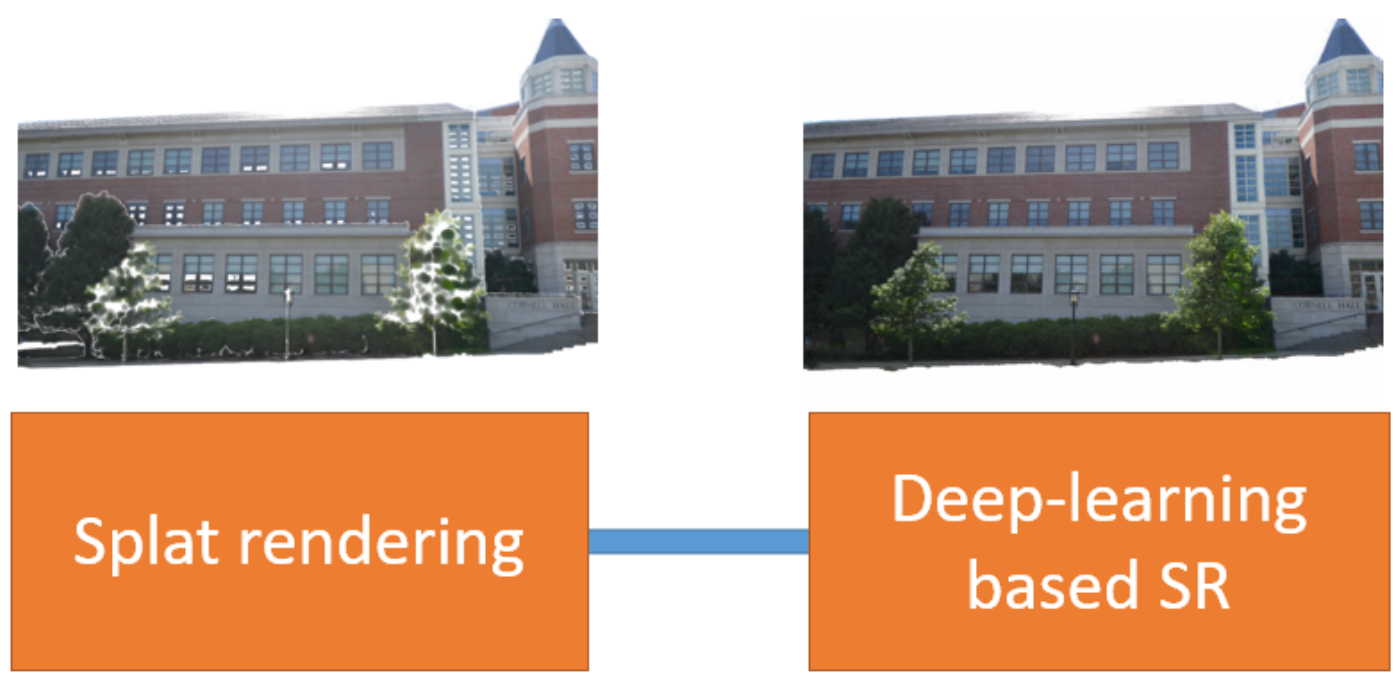

Figure 5.2: Proposed method pipeline.

\subsubsection{Input data}

There are two common ways to obtain a color point cloud of a 3D reconstructed scene. A traditional approach is to use SfM [111] to generate a point cloud from a collection of densely sampled images [112]. Another common approach is to use LIDAR laser scanners that produce highly accurate coordinate measurements. The RGB-color information can either come from imagery collected at the time of the LIDAR survey. By placing a laser scanner at several sample locations, a large-scale 3D color point cloud can be obtained with relative ease.

\subsubsection{Splat rendering}

Point-based splat rendering has been proven to be a flexible and efficient technique for rendering $3 \mathrm{D}$ objects due to its simplicity. The key idea is to approximate a surface by piece-wise planar ellipses, or splats, in the object space and render them to the image space. This technique is widely used in the literature and often needs an associated normal, a color, and a radius $r_{i}$ for each point $p_{i}$ to render the points as small discs. That information can be obtained from neighbors around pixels. 
There are two common approaches to obtain this information, radius search and $k$ nearest neighbors search (KNN). Both can be efficiently retrieved using a $k$-d tree. However, radius search is not suitable for the case of LIDAR data due to its nonuniform distribution. The points tend to be very dense on surfaces close to the scanning location and become sparse on ones further away. In contrast to radius search, KNN is a naturally adaptive method better suited for this situation. Once the neighborhood information is obtained, the point cloud's normals can be approximated by Principal Component Analysis (PCA) which selects an eigenvector corresponding to the smallest eigenvalue of the covariance matrix [113].

Next, we estimate a radius for each point. Since the point normal is assigned to that of the plane it belongs to, we still have to determine the radius $r$ so that we can render a gap-free surface. We do not want it to be too big as this will likely require many overlapping regions be rendered which is computationally inefficient. We propose a heuristic approach which is similar to [100]. First, for each sample point $\left(p_{i}, n_{i}\right)$, we determine the $k$ nearest neighbors using KNN and define $R$ as the furthest distance from a neighbor to the sample point. Therefore, the formed circle at $p_{i}$ with radius $R$ covers the whole $k$ nearest neighbors. In the second step, we project all neighbors whose normals are consistent to that of the sample point into the circle. Lastly, we divide the circle of projected points into 12 sectors and for each non-empty sector, we only keep the closest projection point. We set the splat radii $r$ to be the scale factor of 0.7 of the maximum distance of those points. Although computing the splat radius depends on how many of the nearest neighbors are considered, we have found that it is not sensitive to parameter $k$. Empirically we set $k=50$ for all models in the dataset.

Since each splat is a solid color (determined from its center point), sharp edges may exist between splats. Following [100], we implement a 3-pass rendering technique to blend the color and normal between neighboring splats. In the first pass called 
visibility splatting, the splats are rendered without color information in order to fill the depth buffer. In the second blending pass, after the object is slightly shifted towards the viewer by $\epsilon$, a simple depth test is used to remove all the splats that are far behind the visible surfaces. For each virtual image pixel, the second pass sums up the colors and normals of the splats that lie in the proximity of the visible surface and perspectively project onto the respective pixel. Finally, the normalization pass normalizes the sum-up colors and normals by dividing them by the number of splats projecting onto this pixel. Although the 3-pass rendering technique can give visually pleasant rendering images, it smooths out the gradient information and hence blurs the image due to the use of blending techniques. In what follows, we will describe the use of a deep-learning based approach as a post-processing step to alleviate these unwanted effects.

\subsubsection{Deep-Learning based Super-Resolution with Genera- tive Adversarial Network}

Following Goodfellow et al. [105], in the context of generative adverisial networks, we solve the adversarial min-max problem:

$$
\begin{aligned}
\min _{\theta_{G}} \max _{\theta_{D}} \quad E_{I}^{H R} \sim p_{\text {train }}\left(I^{H R}\right)\left[\log D_{\theta_{D}}\left(I^{H R}\right)\right]+ \\
E_{I}^{L R} \sim p_{\text {train }}\left(I^{L R}\right)\left[\log \left(1-D_{\theta_{D}}\left(G_{\theta_{G}}\left(I^{L R}\right)\right)\right]\right.
\end{aligned}
$$

where $G_{\theta_{G}}$ and $D_{\theta_{D}}$ are generator and discriminator networks which are parameterized by $\theta_{G}$ and $\theta_{D}$ respectively. In our problem, the generator network $G$ is the network to predict a HR image given a LR image. The general idea behind this formulation is that it allows one to train a generative model $\mathrm{G}$ with the goal of fooling a differentiable discriminator $D$ that is trained to distinguish super-resolved images from real images. That is to say, with this approach our generator can learn to predict outputs that are 
highly similar to real images and thus difficult to classify by $D$.

\section{Generator network structure}

Our network structure, named deep network for super-resolution with deeply supervised nets $(S R D S N)$, is illustrated in Fig. 5.3. The network takes a splat rendering of the LR image as input and progressively predicts the intermediate HR image. We supervise all the intermediate results to alleviate the effect of vanishing/exploding gradients. Our network contains two parts: (i) cascade network and (ii) deeply supervised network.

Cascade network: The network has multiple similar structure blocks. Each block, consisting of $d$ convolutional layers followed by a PReLU layer except for the last one, takes an image ( 1 or 3 channels) to produce an intermediate HR image. This intermediate result is the input to the next block and is supervised by a deeply supervised network.

Deeply supervised network: We supervise all the outputs of the network blocks using a deeply supervised nets (DSN) structure [63]. The DSN can be considered as a network regularization that informs intermediate layers about the final objective, rather than relying on the final layer to back-propagate the information to its predecessors. A similar idea of supervising intermediate layers for a convolutional neural network can be found in [62] and [88].

Generator output: Denote $x$ as the input of low-resolution image, and $\hat{y}_{m}, m=$ $1,2, \ldots, M$, as predicted $M$ intermediate outputs. The output of the generator net-

work is averaged over all the intermediate outputs $\hat{y}=\sum_{i=1}^{M} \alpha_{i} \hat{y}_{m}$ with $\alpha_{i}$ indicates the relative importance of the intermediate output, e.g. setting $\alpha_{m}=0,1 \leq m<M$ makes the model turn into a predictor with only a single output at the top. 


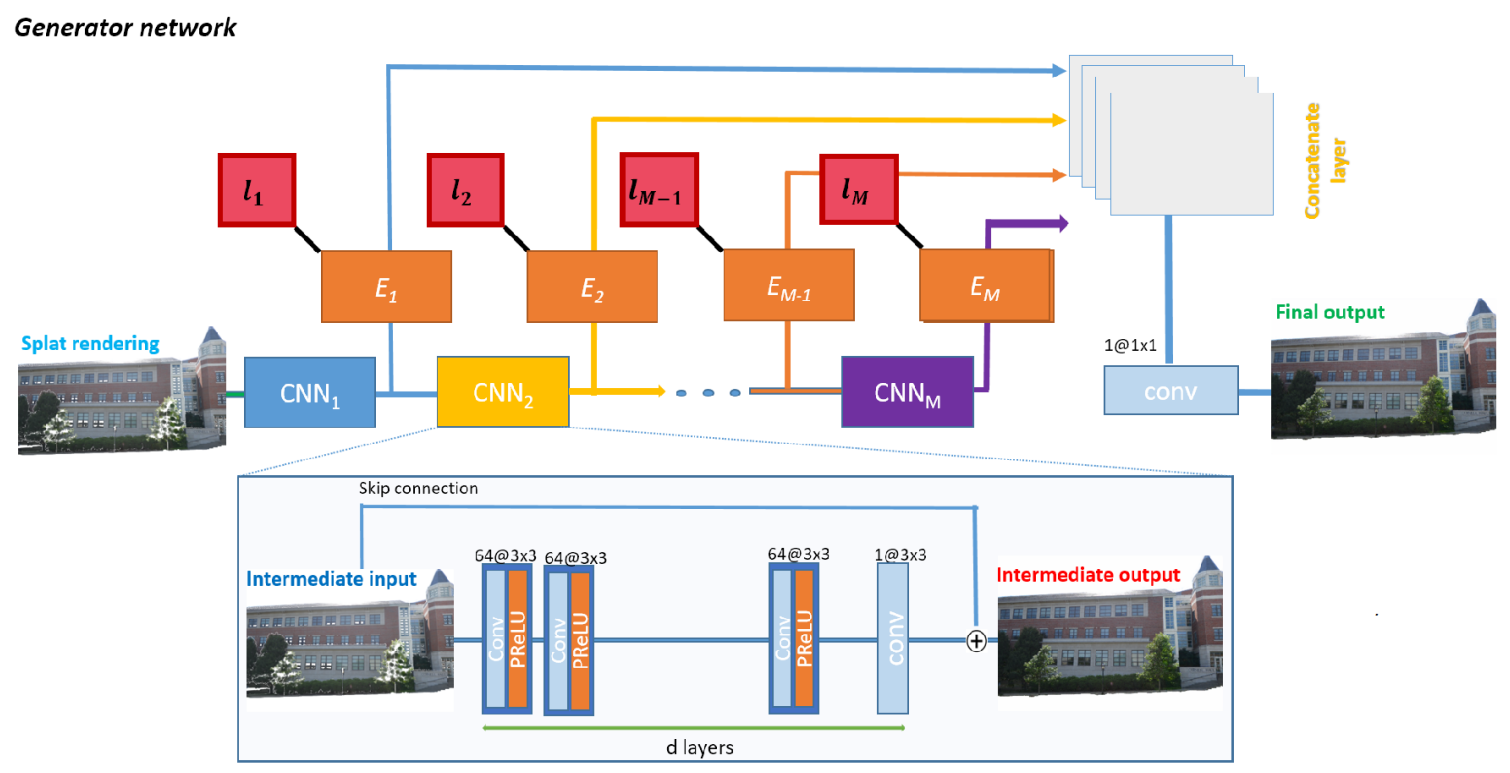

Discriminator network

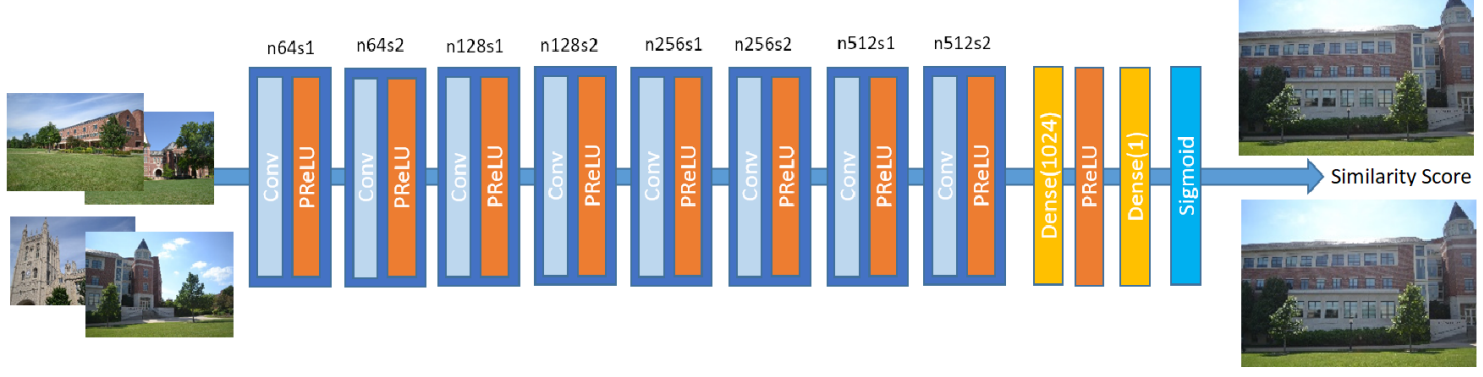

Figure 5.3: (Top) Generator Network Structure. The network consists of multiple blocks with deep supervision, every intermediate result is penalized by a Euclidean loss function $l$. The layout of each network block has a corresponding number of feature maps followed by kernel size indicated for each convolutional (e.g. 64@3x3 stands for $643 \times 3$ kernels). Our final network consists of four blocks $(M=4)$ and 20 layers $(d=20)$ within each block. (Bottom) Discriminator Network Structure with corresponding number of feature maps (n) and stride (s). The network takes a set of natural images to measure the similarity of the generator output image and ground truth image. 


\section{Adversarial Network Architecture}

For the discriminator network, we modify the network proposed by Ledig [101] to simplify the network architecture. We remove the batch normalization layers from the network as Lim et al. [103] presented in their work. It is better to remove them since batch normalization layers get rid of range flexibility from networks by normalizing the features. We also replace the LeakyRELU layers by PReLU layers to allow the network to adaptively learn the coefficients of negative parts. The network consists of 8 convolution layers with increasing feature depths with factors of 2 and decreasing the feature resolutions each time the feature depth is doubled. The discriminator network is trained to solve for the min-max optimization problem described in Equation 5.1.

\section{Loss function}

Given training pair images $\left\{\left(x_{n}, y_{n}\right)\right\}_{n=1}^{N}$, our goal is to optimize both the generator network $G_{\theta_{G}}$ and the discriminator network $D_{\theta_{D}}$ simultaneously. Similar to [101], our loss function consists of a mean square error (MSE) loss (Eq. 5.2), VGG [110] loss (Eq. 5.3) and adversarial loss components (Eq. 5.4).

$$
\begin{gathered}
l_{M S E}\left(\theta_{G}\right)=\frac{1}{N} \sum_{n=1}^{N}\left\|G_{\theta_{G}}\left(x_{n}\right)-y_{n}\right\|^{2} \\
l_{V G G / i, j}\left(\theta_{G}\right)=\frac{1}{N} \sum_{n=1}^{N}\left\|v g g_{i, j}\left(y_{n}\right)-v g g_{i, j}\left(G_{\theta_{G}}\left(x_{n}\right)\right)\right\|^{2}
\end{gathered}
$$

where $\operatorname{vgg}_{i, j}($.$) is the feature map obtained by the j-t h$ convolution before the $i-t h$ max-pooling layer within the VGG network.

$$
l_{\text {Gen }}\left(\theta_{D}\right)=\sum_{n=1}^{N}-\log D_{\theta_{D}}\left(G_{\theta_{G}}\left(x_{n}\right)\right)
$$


The final objective function needs to be minimized is

$$
\mathcal{L}\left(\theta_{G}, \theta_{D}\right)=\alpha_{1} l_{M S E}\left(\theta_{G}\right)+\alpha_{2} l_{V G G / i, j}\left(\theta_{G}\right)+\alpha_{3} l_{G e n}\left(\theta_{D}\right)
$$

where $\alpha_{i}(i=1,2,3)$ are given weighting parameters. Empirically, we choose $\alpha_{1}=1$, $\alpha_{2}=1.0 e-5$ and $\alpha_{3}=1.0 e-3$.

\subsection{Experiments}

\subsubsection{Training Dataset}

Deep learning techniques usually require many pairs of LR and HR images for training. In our problem, the LR images are 3-pass splat rendering (splat rendering for short) images whereas the HR images are texture mesh images. We synthesize the LIDAR scans on the 3D mesh models where we can control the location, rotation angle and focus of the LIDAR and camera. In this work, we use 78 mesh models from Google SketchUp. To simulate the LIDAR scan, we first normalize a model so that it can be fit in an unit sphere and set a point on that sphere and in front of the main facet of a building as the virtual LIDAR scanner's position. We create a grid of cells by sub-dividing the space based on the polar and azimuthal angles of the spherical coordinates ${ }^{1}$ where rays are cast from the LIDAR's position. We keep the first intersection between the rays and the mesh model as a 3D point along with color.

In addition to using the synthetic dataset, we also evaluate the method on a real LIDAR dataset. We used a Leica laser scanner to capture a large scene along with photographs on a university campus.

\footnotetext{
${ }^{1}$ Note the sub-dividing in the spherical coordinates (which is used in real LIDAR) is the primary reason for the produced scattered point clouds because there is a higher density in the center region than in the outer region.
} 
Next, we need to generate a set of pairs of LR-HR images which are 3-pass renderings and mesh images respectively. For the synthetic LIDAR data, we use a technique similar to [114] which is used to generate camera locations along with parameters around the 3D model. For each location, we render the point model with splats and keep its rendering as the LR image. We then render the corresponding mesh model and keep its rendering as the corresponding HR image. For the real data, we use the natural images as HR images, and generate splat images with the same camera parameters used in the HR image. In total, we created a dataset consisting of 2000 image pairs of synthetic data and 200 image pairs of real data. Both synthetic data and real data are used for training but we keep two types of data for testing. Set 1 contains 100 synthetic image pairs and Set2 contains 20 real image pairs. All the images have the same size of $768 \times 1024$.

\subsubsection{Implementation details}

Data samples: To prepare the training data, we sample 400K patches from splat rendering images with a stride of 10 and crop the corresponding HR patches from the ground truth images. For a synthetic dataset, it is trivial to crop a corresponding HR patch of a LR patch since we can control the camera parameters of virtual views. However, we can not do the same for real datasets due to the numerical error involved when recovering the camera's intrinsic and extrinsic parameters of natural images. To overcome this issue, for each image, we recover the rotation and translation, and then generate the splat and texture splat images. Since the texture splat rendering has high quality, we can do dense-SIFT matching between the texture splat image and the natural image. We extract all the patches at the matched SIFT feature locations with SIFT's orientations and scales in both the splat images and the natural images to form image pairs for training.

Data augmentation: Inspired by $\left[\begin{array}{c}94 \\ 98\end{array}\right]$, we augment the training data in two 
ways: (i) Rotation: rotate images by $90^{\circ}, 180^{\circ}$ and $270^{\circ}$; (ii) Flipping: flip images horizontally or vertically. These steps together lead to an augmented training set that is a factor of 8 times larger than the original data set.

Training details: We implement our network using the deep learning framework TensorFlow [115]. For simplicity, we represent our proposed $S R D S N$ network as $\operatorname{SRDSN}(d, M)$ where $d$ is the number of convolutional layers in a block and $M$ is the number of blocks in the model. We first train the SRDSN with MSE loss. Empirically, we choose the following hyper-parameters: batch size (64), patch size (48), convolutional filters and bias randomly initialized as described in [90]. We adopt the adjustable gradient clipping [60] to boost the convergence rate while suppressing

exploding gradients. Specifically, the gradients are clipped to $\left[-\frac{\theta}{\gamma} ; \frac{\theta}{\gamma}\right]$, where $\gamma$ is the current learning rate and $\theta=0.01$ is the gradient clipping parameter. Furthermore, we use the Adam optimizer [97] with an initial learning rate of $10^{-4}$. Next, we train SRDSN-GAN. We increase the patch size to 244 and reduce the batch size to 8 due to the GPU memory limitation. All the other parameters are kept the same as the SRDSN. Training takes four days on a PC using a Nvidia TitanX GPU.

\subsubsection{Comparison with state-of-the-art rendering techniques}

For quantitative comparison, we use the peak signal to noise (PSNR) and the structural similarity (SSIM) indices. As mentioned in Section 5.4.1, it is trivial to compute those indices for the synthetic testing set for comparison. For real datasets, we follow the same methodology of generating real training datasets. To compute $P S N R$ and SSIM of a rendered image with its corresponding natural image, we perform DenseSIFT feature matching. We extract the patches on both of the images at matched locations and compute their PSNR and SSIM respectively. The PSNR and SSIM of the two images are the average of all PSNR and SSIM at patch level. Table 5.1 shows PSNR and SSIM indices on the designed testing sets. For synthetic testing 
Figure 5.4: Comparisons with other methods on synthetic testing set (Set1) for the three examples. From left to right and top to bottom: OpenGL Point Rendering, Texture Mesh, Intensity Completion, Gradient Completion, Splat Rendering, Texture Splat, SRDSN, and SRDSN-GAN.

set Set1, texture splat rendering obtains an average PSNR of 33.18 and an average SSIM of 0.96, SRDSN 33.35 and 0.97 whereas SRDSN-GAN 33,57 and 0.97. For the real testing set $S e t 2$, although the texture splat image can render quite well at high texture regions, its performs worse than $S R D S N-G A N$ in terms of $P S N R$ and SSIM (26.24 and 0.86) because it cannot fill the holes in the data. Both the SRDSN and $S R D S N-G A N$ can fill holes in the image since they learn missing information from the training data. The average PSNR and SSIM of SRDSN-GAN on Set2 are 27.32 and 0.89 whereas ones of $S R D S N$ are 25.44 and 0.84 . Some visual examples of the two testing sets are shown in Fig. 5.4 and Fig. 5.5.

Table 5.1: Quantitative comparison of the approaches on the testing sets.

\begin{tabular}{|r|r|r|r|r|}
\hline Method & \multicolumn{2}{|c}{ Set1 } & \multicolumn{2}{c|}{ Set2 } \\
& PSNR & SSIM & PSNR & SSIM \\
\hline Splat rendering & 23.5 & 0.84 & 22.98 & 0.78 \\
IC [100] & 24.78 & 0.89 & 22.72 & 0.78 \\
GC [100] & 28.56 & 0.96 & 25.12 & 0.84 \\
Texture splat rendering & 33.18 & 0.96 & 26.24 & 0.86 \\
SRDSN & 33.35 & 0.97 & 25.44 & 0.84 \\
SRDSN-GAN & 33.37 & 0.97 & 27.32 & 0.89 \\
\hline
\end{tabular}


Figure 5.5: Comparisons with other methods on real testing set (Set2) for the three examples. From left to right and top to bottom: OpenGL Point Rendering, Photograph, Intensity Completion, Gradient Completion, Splat Rendering, Texture Splat, $S R D S N$, and SRDSN-GAN.

\subsubsection{Interactive 3D Scene Navigation}

The proposed method can be used to support interactive navigation of large scale $3 \mathrm{D}$ scene represented by colored point clouds. Since the generator network (SRDSN) is quite deep (up to 80 layers), it cannot render every frame in real-time. We have designed a novel rendering pipeline to avoid this issue. Imagine that we are navigating a very large point cloud such as an urban scene. Instead of doing super-resolution for every frame, we just do super-resolution on several synthetic views which cover the whole scene. The high-resolution versions of those synthetic views are kept as texture images along with the Model, View and Projection matrices (known as MVP). Using those matrices and texture images, we can perform texture splat rendering which can be done in real time using ordinary computers. We repeat this process every time users enter a new scene. Single frame super-resolution is very fast with modern GPU's as shown in Table 5.2. Moreover, this process can be done off-line which is transparent to users. Thus, the cost of rendering is reduced to the cost of texture splat rendering. Table 5.3 shows more details of rendering times for different sizes of point clouds.

Table 5.2: Running time of the proposed method with different image sizes

\begin{tabular}{|r|r|r|r|r|}
\hline & $480 \times 640$ & $768 \times 1024$ & $1080 \times 1920$ & $2016 \times 3940$ \\
\hline Running time $(\mathrm{ms})$ & 259 & 780 & 1356 & 4324 \\
\hline
\end{tabular}


Table 5.3: Rendering time

\begin{tabular}{|r|r|}
\hline Number of points & fps \\
\hline $500 \mathrm{~K}$ & 67 \\
$1000 \mathrm{~K}$ & 40 \\
$2000 \mathrm{~K}$ & 36 \\
$3000 \mathrm{~K}$ & 30 \\
$4000 \mathrm{~K}$ & 24 \\
\hline
\end{tabular}

\subsubsection{Image matching}

In this section, we demonstrate the ability to extract SIFT features [116] for matching from generated synthetic images. Since we have all the mesh images of all the SketchUp models, we choose 5 mesh images for each model to match with the synthetic images. In total, we have 390 mesh images for the synthetic data. For the real LIDAR data, we chose 10 images taken from a variety of devices: Nikon D90, Nikon D3100, and cell phones to form the real dataset.

Similar to [100], we measure the average numbers of SIFT features extracted from the images. In order to measure the repetitiveness and the distinctiveness of extracted features, we use the SIFT ratio test: two SIFT features $f_{1}, f_{2}$, one in a mesh image and one in a synthetic image, are considered as a match if their descriptors pass the SIFT ratio test $\left\|\operatorname{des}\left(f_{1}\right)-\operatorname{des}\left(f_{2}\right)\right\|<0.7 *\left\|\operatorname{des}\left(f_{1}\right)-\operatorname{des}\left(f_{2}^{\prime}\right)\right\|$ for all features $f_{2}^{\prime}$ also extracted in the synthetic image. We match each query photo against all synthetic views rendered from the same scene. For each feature on a synthetic view, we back project to the 3D point cloud. The resulting 2D-3D correspondences are then used to estimate the pose of each query image. The Avg. Matches of Table 5.4 describe the average percentage of matches and the Avg. Inliers columns describe the average percentage of SIFT features that are used to compute the camera pose position. 
Table 5.4: Feature extraction evaluation

\begin{tabular}{|r|r|r|r|r|r|r|}
\hline \multirow{2}{*}{ Method } & \multicolumn{3}{|c|}{ Set1 } & \multicolumn{3}{c|}{ Set2 } \\
\cline { 2 - 7 } & $\begin{array}{r}\text { Avg. No } \\
\text { of SIFT }\end{array}$ & $\begin{array}{r}\text { Avg. } \\
\text { Matches }\end{array}$ & $\begin{array}{r}\text { Avg. } \\
\text { Inliners }\end{array}$ & $\begin{array}{r}\text { Avg. No } \\
\text { of SIFT }\end{array}$ & $\begin{array}{r}\text { Avg. } \\
\text { Matches }\end{array}$ & $\begin{array}{r}\text { Avg. } \\
\text { Inliners }\end{array}$ \\
\hline Splat rendering & 1224 & 28.26 & 10.62 & 2845 & 13.67 & 8.45 \\
IC [100] & 1135 & 31.36 & 10.38 & 2754 & 12.93 & 9.34 \\
GC [100] & 1345 & 43.40 & 19.41 & 3255 & 34.83 & 14.45 \\
Texture splat & 1456 & 61.91 & 21.35 & 3524 & 43.53 & 18.53 \\
SRDSN & 1554 & 64.74 & 23.49 & 3552 & 47.91 & 19.45 \\
SRDSN-GAN & 1573 & 66.24 & 23.94 & 3785 & 48.01 & 19.85 \\
\hline
\end{tabular}




\section{Chapter 6}

\section{Summary and concluding remarks}

\subsection{Future work}

Depending on the type of data used, our pipeline may face a few roadblocks. If only a small percentage of the identified ensemble features are actually inliers, it is very unlikely that we will find a correct image alignment using RANSAC. To address this in the future, we will explore using techniques that have expanded on RANSAC to work with extremely noisy data such as [117].

One of the reasons we may encounter this problem is that fact that, currently, our pipeline does not incorporate affine invariant descriptors and is limited to relatively small baselines. One option for addressing this is to include an affine invariant descriptor such as ASIFT [16] to expand our work. Any keypoint matching technique or combination of techniques can be inserted into this pipeline.

One of the main bottle necks of our pipeline is the requirement that the image be rectified. While this is an acceptable assumption for urban images, we cannot guarantee that the method we use will also be able to perfectly rectify each image. Other repetition matching pipelines use an initial estimation of the repetition to refine 
rectification $[50,51]$. We can apply a similar approach to our work. We also assume that the images do not have severe radial distortion. Images that do have this type of distortion may not be rectified correctly. A radial undistortion step could be applied in this case [38]. The effect that radial distortion has on our evaluation is discussed in greater detail in the Supplementary Material.

This pipeline can easily be expanded to handle images in which multiple buildings of a facade are visible. Multiple rectified versions of a photograph that are each arranged to have a different building plane be front facing can be matched to a query image. We can also alternate between collapsing an image along the horizontal direction to collapsing it along the vertical direction and computing a 1-D horizontal transformation first. This may be a good option for data of high-rise buildings if the majority of repetitive features lay along columns instead of rows.

For SISR, our propoed network, SRDSN, uses bicubic interpolation image as its input, which has some trade-offs. The network can support multiple scale factors as shown in Section 4.4.4. However, it is not efficient due to the fact that working on HR space requires more memory and computation. In the future work, we plan to investigate a network which works directly on a LR image instead of an interpolation image and uses upsampling operators in a later stage.

\subsection{Conclusion}

In this thesis, we have presented a contextual framework for describing and matching keypoints neighborhoods for the purpose of aligning image pairs. Our framework can be used to build upon and complement local keypoint matching techniques, creating an ensemble feature, or can be used independently to describe and match keypoints. In addition to potentially incorporating raw local keypoint matches provided by existing techniques, we can use various keypoint detectors as anchors to study larger 
regions surrounding keypoints in terms of salient line segments and texture-rich HOG information. We have studied, tested, and presented results for using our framework in combination with several different keypoint descriptors, including our newly proposed Pseudo Corners, to demonstrate that it is very flexible and not dependent on any one method or type of information. By using both local and regional descriptors, we are able to identify small unique regions surrounded by clutter and to clear up potential ambiguity during feature matching by looking at a larger subset of the image. This pipeline has been designed to combine these various feature extraction and matching methods in such a way that it is robust under lighting, content, and rendering changes. We have shown quantitatively that using this information can increase the accuracy of identified matches and image alignment over using SIFT alone as well as can be used to accurately describe Harris-Laplacian corners and our proposed Pseudo Corners. Our technique can work on images containing a variety of architectural styles, man-made features, and natural content without making assumptions about the general structure of a scene and can handle different artistic representations of the same location.

We have also presented a method for registering images of architecture that contain repetitive features that have a tendency to create ambiguity for general keypoint matching methodologies. In contrast to several state-of-the-art approaches to registering urban data, our method does not try to fit an evenly-spaced grid to repetitive elements [2], considers both salient and repetitive regions simultaneously [17], and does not require that the same portion of a buildings facade be captured in different images [18]. Our method takes a twostep approach to align images one dimension at a time to reduce the search space during each matching stage. We have discussed how we perform a dimension reduction amongst unorganized repetitive features to further remove ambiguity from the search space when matching regions across images. We have also proposed equations for determining the saliency of matching regions between 
an image pair without requiring machine learning techniques to learn what elements are salient on a large dataset [25]. We believe that by developing a pipeline without these constraints, our method is more robust than many other proposed registration approaches, allowing us to align challenging, ambiguous architectural photographs in a more general manner.

For SISR, we have proposed a single image super-resolution with deeply supervision nets and incremental learning. Our model progressively predicts intermediate high-resolution images and combine them to obtain the final high-resolution result. By cascading multiple similar structure blocks and optimizing the network with an incremental learning strategy, our proposed SRDSN alleviates the common difficulty of training a very deep network. Quantitative evaluations on benchmark datasets demonstrate that the proposed model performs favorably against the state-of-the-art SR algorithms in terms of visual quality.

For point-based rendering, we have proposed a novel framework for pointbased rendering by incorporating the deep learning based superresolution technique with point rendering methods. The network takes a splat rendering image as input and generates the corresponding high-qualtiy image. Our network which is based on the deeply supervised stacked model with generative adverisial network has demonstrated superior performance in terms of accuracy compared to other methods. To generate a photo realistic rendering of the real 3D world is a holy grail in computer graphics and remains a challenge even after several decades of research. Our proposed method is a small step in this direction. In the future we would like to build on the idea proposed in this paper, i.e. using realistic high quality natural images as the ground truth data to train the neural network to learn to generate rendering images indistinguishable from the real images. Currently, the proposed method is working in the 2D image space. We would like to extend the proposed network to work directly in 3D and be able to generate high resolution 3D point clouds using low resolution colored point clouds 
and single and/or multiple high quality images as input. That would be very useful for $3 \mathrm{D}$ scene modeling. Another direction we would like to pursue is to investigate shallower network that can achieve similar results but with a much faster speed so that it can generate the output in real-time. 
Appendix A

Supplementary Material for An Ensemble Approach to Image Matching Using Contextual Features 


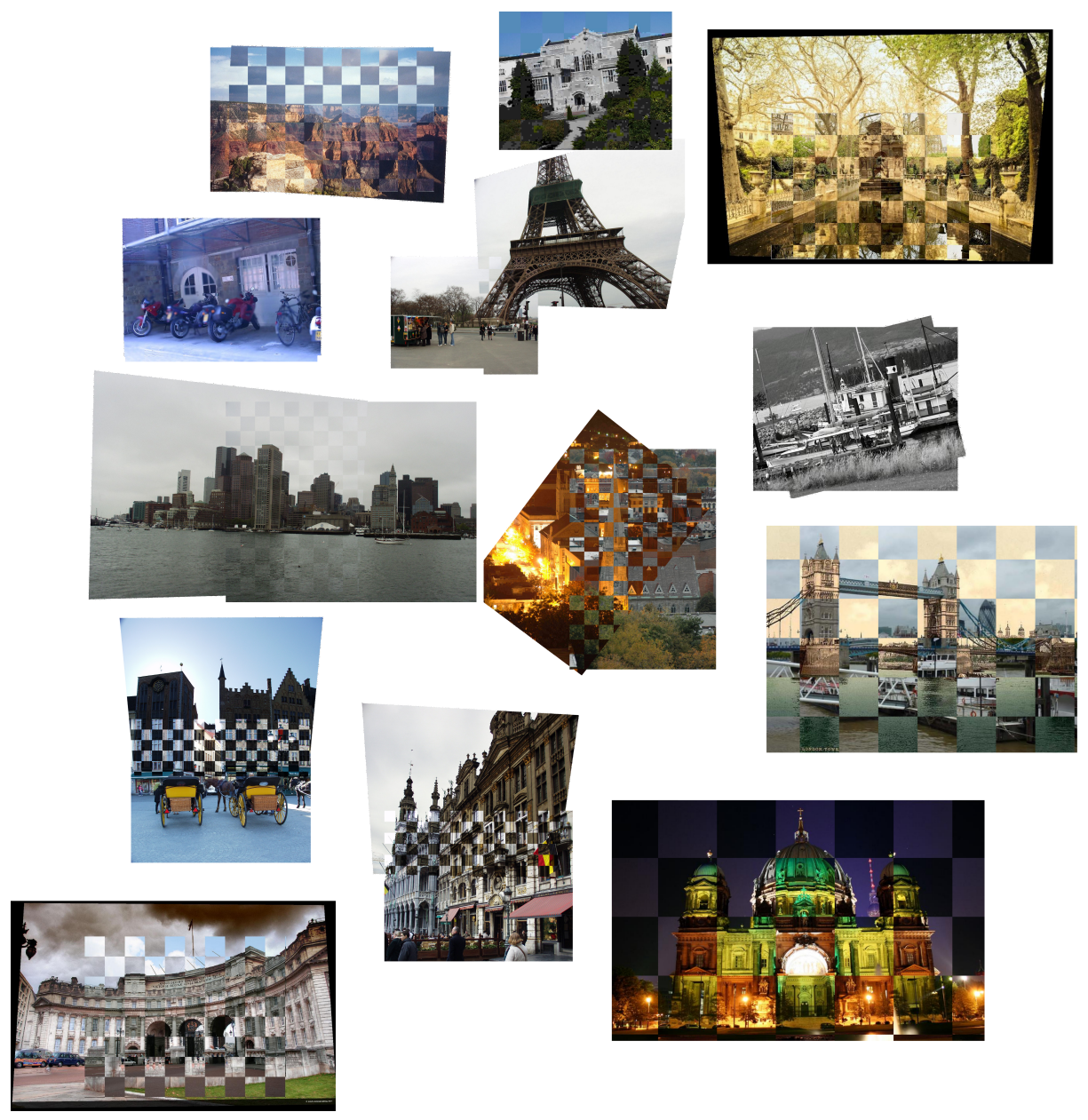

Figure A.1: Registration results after applying our pipeline to several public dataset image pairs presenting challenges including small image overlap, camera rotation, lighting changes, and .JPEG compression. Images provided courtesy of [3], [7], [1] and $[2]$.

\section{A.1 Comparison with Other Methods}

We compared each of our three matching techniques to other matching methods including MSER with SIFT descriptors, SIFT Flow [9], and local symmetry features [2] on the symmetry dataset. Following the local symmetry paper, we tried matching the images using only symmetry descriptors and symmetry and SIFT descriptors concatenated. These results are shown in Figure A.4. A few visual examples from this test are displayed in Figure A.3. 


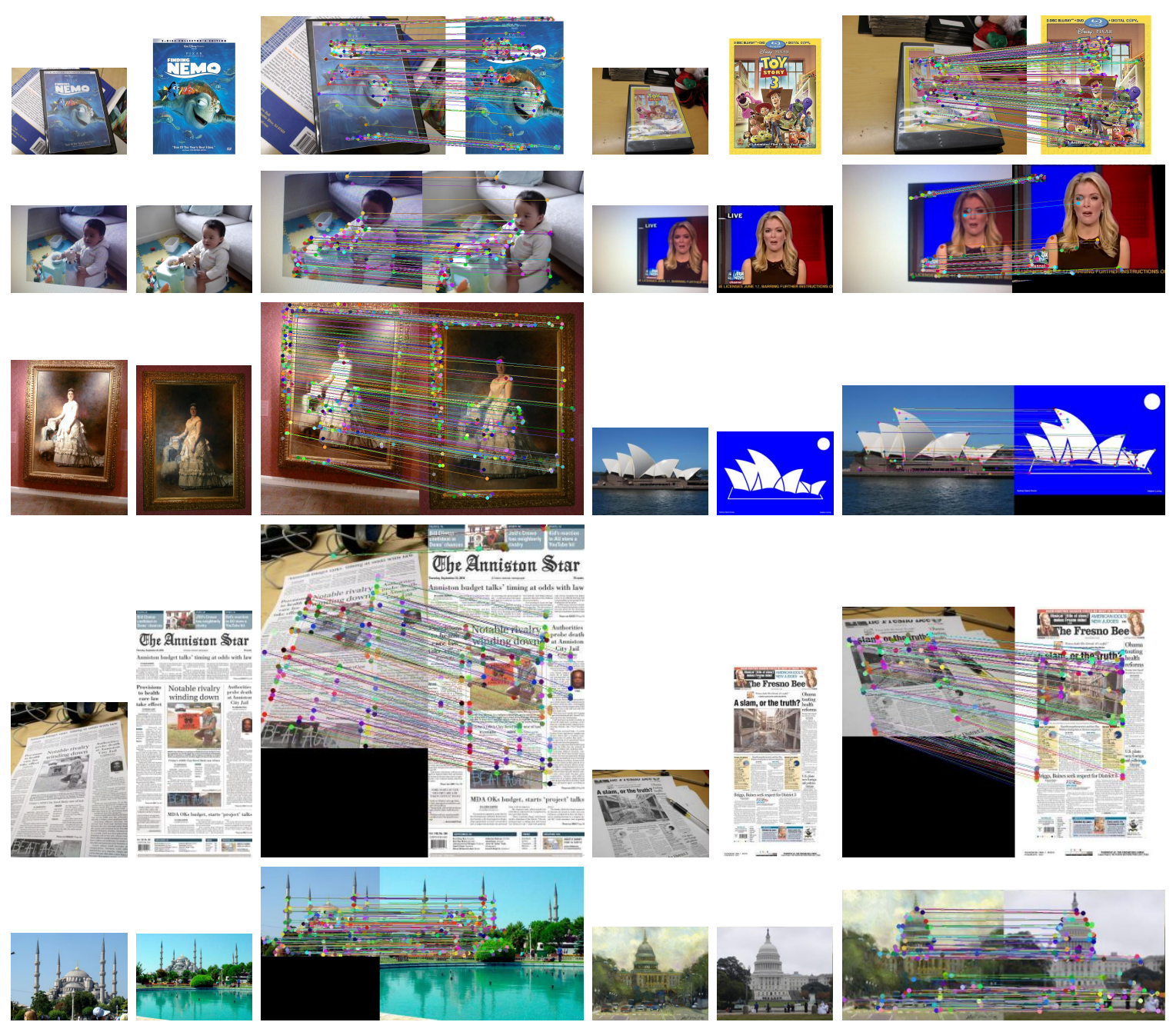

Figure A.2: Keypoint matches for images from Stanford Mobile Visual Search dataset [8] and the dataset provided in [1].

\section{A.2 True Positive/False Positive Rates}

We created several non-matching image pairs from various categories of the Stanford Mobile Visual Search dataset [8] to test the true positive and false postive rates of our methods. We used images from the museum paintings, dvd covers, print, landmarks, and video frames sub-datasets. We chose two pairs of query and reference images from each dataset and matched each query image to the ten selected reference images. If the most geometrically verified matches (using a solved homography) were found between the correctly matching query and reference pair, the image was marked 111 

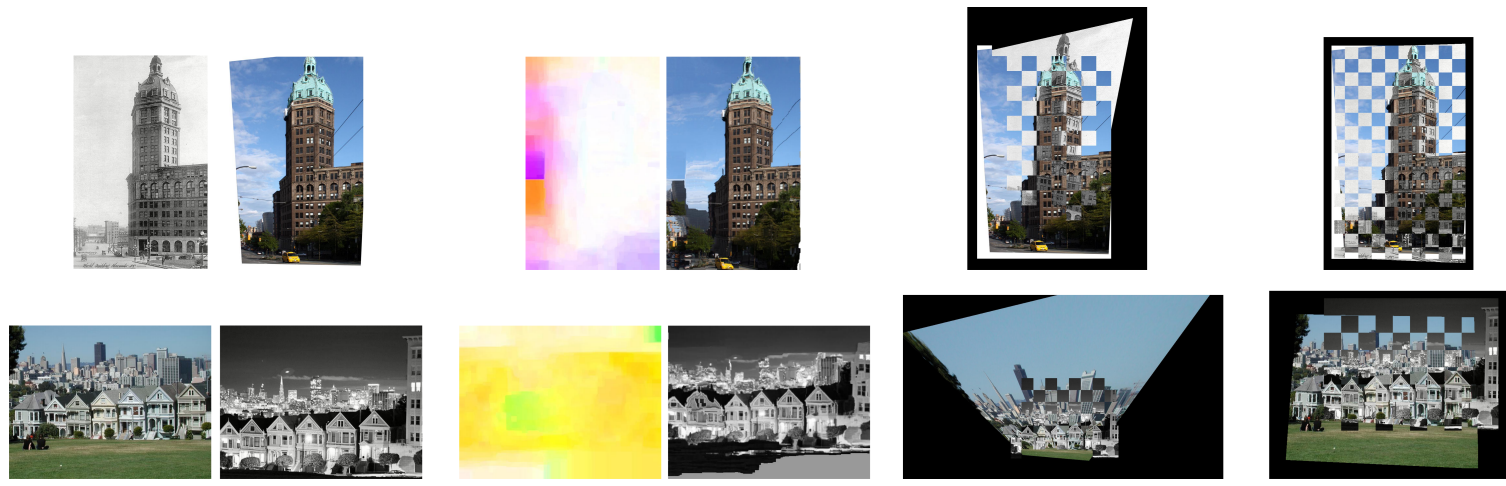

Figure A.3: Registration results after applying our pipeline to several symmetry database pairs. Images provided courtesy of [2]. Top: worldbuilding, Bottom: paintedladies18. From left to right: The original image pair, SIFT Flow results, stitched images using the SIFT+Symmetry method described in [2], and stitched images using our pipeline. For the SIFT Flow results, we show the colored pixel displacement map on the left and the $I_{2}$ warped to $I_{1}$ 's image plane on the right.

as being a true positive. If a non-matching pair had more matches than a correct matching pair with the same query image, it was marked as a false positive. The results of testing our contextual methods in this manner are shown in Table A.1. We repeated this test focusing on architectural buildings from the MPEG CDVS Landmark Dataset. We chose 60 pairs of images from the the Oxford Buildings Dataset [118], the Zurich Building Image Dataset [119], and the Stanford Mobile Visual Search dataset [8]. Thumbnails of the query and reference image pairs we tested are provided in Figure A.5. These images present obstacles for matching including changes in lighting, perspective, scale, rendering style, and image blurring. Table A.2 shows the the true and false positive rates we obtained after matching each of the 60 selected query images to each of the 60 reference images using our three different contextual approaches.

Table A.1: True Positive/True Negative Rates of Ensemble Methods on Stanford Mobile Visual Search Dataset

\begin{tabular}{c|c|c}
- & True Positive & False Positive \\
\hline Ensemble SIFT & 1.0 & 0.0 \\
Cont. Harris-Lap. & 0.7 & 0.111 \\
Cont. Psu. Corners & 0.8 & 0.1
\end{tabular}




\section{Comparing Different Methods}

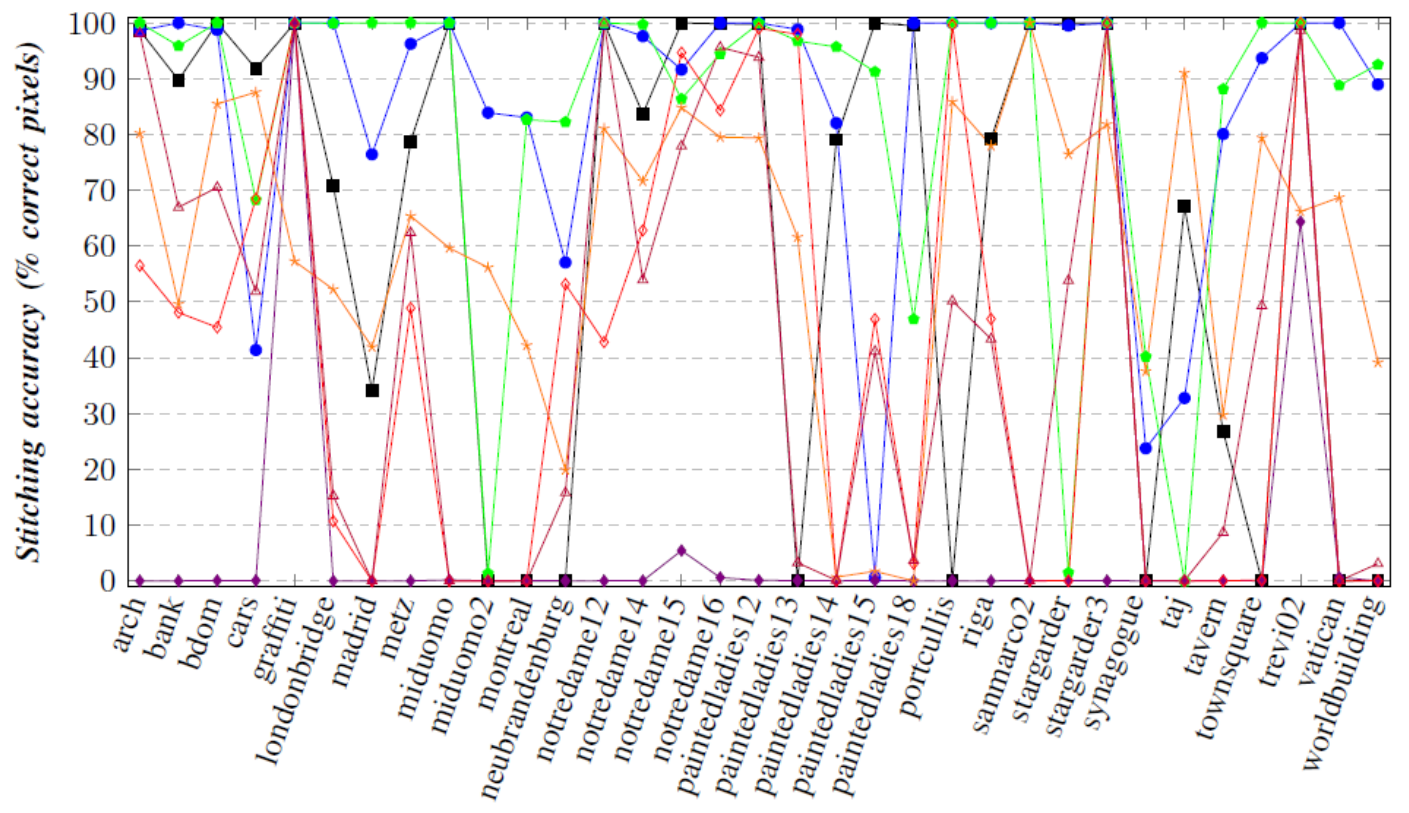

\section{Symmetry Image Pair Name}

$$
\begin{array}{|lc}
-- & \text { Ens. SIFT } \\
-- & \text { Cont. Harris } \\
-- & \text { Cont. Psu. Corners } \\
\rightarrow- & \text { MSER } \\
- & \text { SIFT Flow } \\
- & \text { Symmetry } \\
\rightarrow & \text { SIFT+Symmetry } \\
\hline
\end{array}
$$

Figure A.4: Comparing our ensemble methods to other matching techniques. We show in this chart accuracy measures for aligned image pairs using Ensemble SIFTbased features, Contextual Harris Laplacian features, Contextual Pseudo Corners, MSER with SIFT descriptors, SIFT Flow [9], and Local Symmetry Features [2]. For the Local Symmetry Features method, we tested using just the symmetry descriptor and the symmetry descriptor concatenated with each feature point's SIFT descriptor as described in [2]. 
Table A.2: True Positive/False Positive Rates of Ensemble Methods on Landmark Dataset

\begin{tabular}{c|c|c}
- & True Positive & False Positive \\
\hline Ensemble SIFT & 0.883 & 0.027 \\
Cont. Harris-Lap. & 0.833 & 0.056 \\
Cont. Psu. Corners & 0.833 & 0.084
\end{tabular}




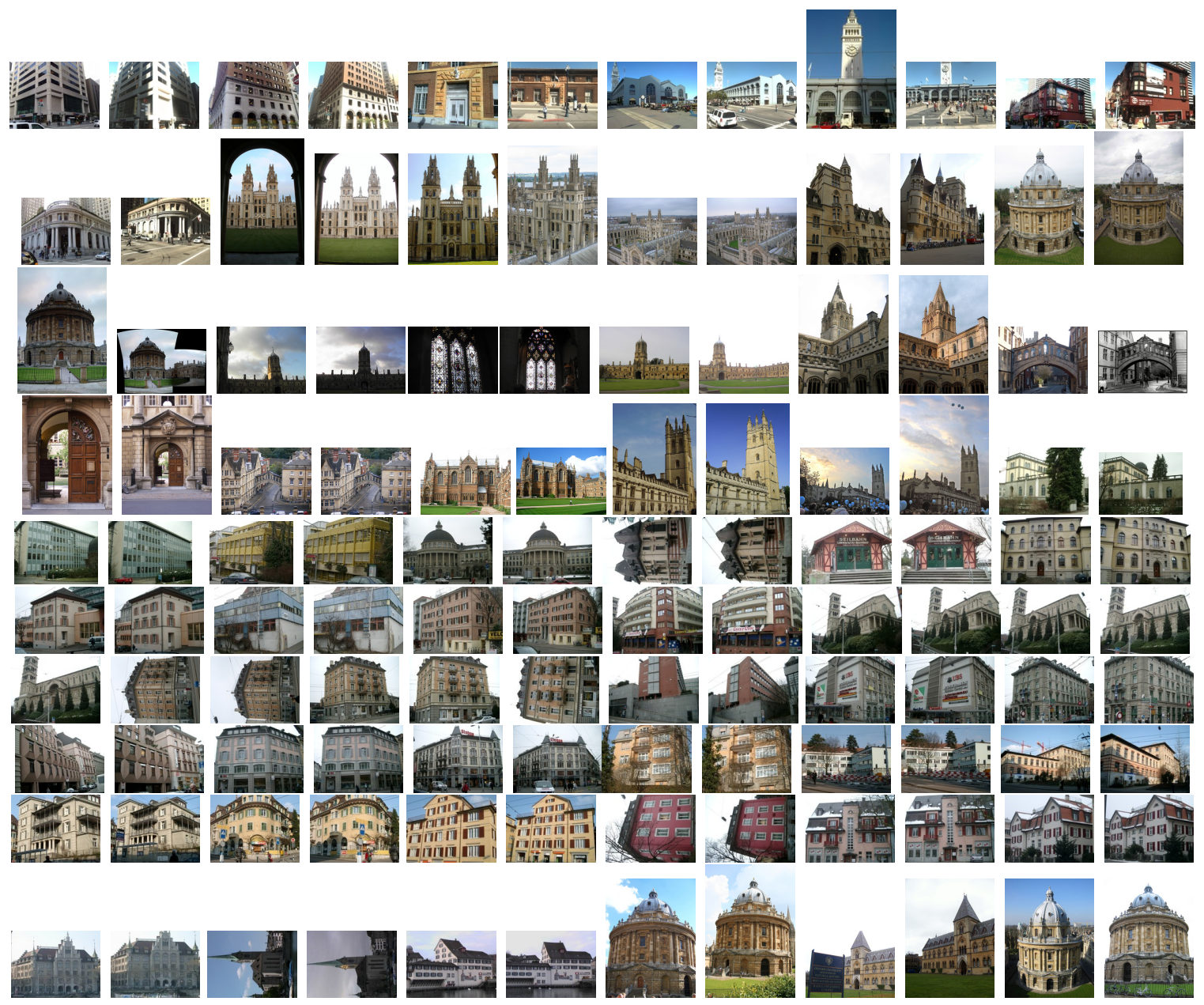

Figure A.5: Image pairs from Landmark dataset that are used for True Positive/False Positive tests in Table A.2. 


\section{A.3 Complexity}

In Table A.3 we have outlined the complexity of different stages of our contextual matching algorithm. We present the complexity in terms appropriate for each stage whether they be number of pixels, lines, features, or matches involved in the method. The complexity of assigning pixels to MSER-based neighborhoods is linear in terms of the number of pixels in the image as each is assigned to a region in addition to the same complexity of actually extracting MSER's [120]. Each pixel is visited once to choose from which level of the Gaussian Pyramid it should choose an MSER. The complexity of extracting line segments using LSD is proportional to the number of pixels in the image as described in [34]. When we extract Pseudo Corners using line segments, we check the intersection of each pair of line segments within a defined distance from each other. The complexity of doing this is at most $O\left(l^{2}\right)$ in terms of the number of lines. To assign line segments to nearby keypoints to create contextual linear descriptors we must compute the distance of each line $l$ to each keypoint feature f. Matching contextual linear descriptors is that of Hungarian Graph Matching which is $O\left(l^{3}\right)$. To construct contextual HOG descriptors, we need to traverse every pixel within a defined neighborhood surrounding each keypoint. The complexity of these descriptors depends on the approach being used for matching. For Ensemble SIFT, contextual descriptors are compared for each potential SIFT match that falls within a specified ratio range, making the matching linear in terms of the number of SIFT matches. For our contextual corner methods, each corner feature $f$ in $I_{1}$ is compared to each corner feature $g$ in $I_{2}$, giving us a complexity of $O(f * g)$. For our ICP refinement, each feature in $I_{1}$ is compared to every feature in $I_{2}$ within a specified ratio, giving this stage, at worst, also a complexity of $O(f * g)$. For our global Levenberg-Marquardt refinement, we check the gradient magnitude at each pixel in $I_{2}$ that has a corresponding line feature in $I_{1}$, making this stage linear in terms of the number of features whose gradients are checked. 
Many of these stages are dependent on each other. For instance, the number of Pseudo Corners we extract for Contextual Pseudo Corner matching and the time it takes is related to the number of line segments we have identified in an image pair. The time for extracting line segments, and likely the number of line segments we have for finding corners, is guided by the number of pixels in, or resolution of, the images.

As the resolution of the image increases, it is likely that we will find more line segments and more keypoints for all three of our Contextual Matching approaches and for image alignment refinement. Since the main stages of our algorithm are dependent on the number of pixels and features in an image, it will generally take our program longer to perform matching on higher resolution images. This increase in runtime and number of features with higher resolution images can be observed in Table A.4. Since higher resolution images tend to contain more features and finer details, our matching pipeline has more information to perform accurate matching than it generally does with lower resolution images.

Table A.3: Complexity of Different Stages of Our Pipeline

\begin{tabular}{|c|c|c|}
\hline Stage & Paper Section with Explanation & Complexity \\
\hline Assigning pixels to MSER-based neighborhoods & II. A. & $O(p)+O(p \log \log p)$ \\
\hline Extracting line segments (LSD) & II. B. and II. F. & $O(p)$ \\
\hline Extracting pseudo-corners & II. F. & $O\left(l^{2}\right)$ \\
\hline Creating linear contextual descriptors & II. B. & $O(f * l)$ \\
\hline Matching linear contextual descriptors (Ensemble SIFT) & II. B. & $O\left(l^{3}\right)$ \\
\hline Creating HOG contextual descriptors & II. C. & $O(f * q)$ \\
\hline Matching HOG contextual descriptors (Ensemble SIFT) & II. D. & $O(m)$ \\
\hline Matching contextual descriptors (Contextual Corners) & II E. and II. F. & $O(f * g)$ \\
\hline Refining with ICP & III. & $O(f * g)$ \\
\hline Refining with Levenberg-Marquardt & III. & $O(f)$ \\
\hline
\end{tabular}

Notation: p - number of pixels in image, q - number of pixels in local neighborhood, 1 - lines, f features (in image 1), $\mathrm{g}$ - features (in image 2), $\mathrm{m}$ - SIFT matches 


\section{A.4 Runtimes}

Table A.4 shows the runtimes of various components of our pipeline. All tests were performed using a Dell Optiplex 7010 desktop with 7.7 GB of memory and a $3.2 \mathrm{GHz}$ Intel Core i5-3470 processor running the Ubuntu 12.04 64-bit operating system. We ran all of our contextual methods on the Symmetry Dataset [2]. The timings for each method and stage in Table A.4 are the average runtime in seconds to complete the stage on all of the images in the dataset. The resolutions of these images range from $640 \times 350$ to $889 \times 1221$. The top left quadrant of Table A.4 shows the timings of stages of our pipeline that are used regardless of the descriptor and matching technique used. The top right shows timings for stages that are used specifically by our Ensemble SIFT method, and the bottom two quadrants show the timings for our Contextual Harris-Laplacian and Pseudo Corner methods. In our Ensemble SIFT method, we compare contextual descriptors of all potential SIFT matches within a specified distinctiveness ratio. The contextual descriptors for a keypoint are only compared to the contextual descriptors for its potentially matching keypoint. For this reason, in the Ensemble SIFT section of the table, we report the average pairwise times to compare two linear contextual descriptors with each other and two HOG contextual descriptors with each other. We also include the total average time to match all of the SIFT features using SIFT descriptors and contextual descriptors according to our ensemble approach. For the Contextual Corner methods, each corner in $I_{1}$ is compared to all the corners in $I_{2}$. For both Contextual Harris-Laplacian and Contextual Pseudo Corners, we have included the average time to find the closest match for a single keypoint in $I_{1}$ in $I_{2}$. We have also documented the total time it takes to complete the matching, including matching all corners in $I_{1}$ to all corners in $I_{2}$. Notice the average times vary between the two Contextual Corner methods in relation to how many corners are identified. We use the OpenCV library [121] to extract SIFT features and to perform matching and compute homographies for all 
Table A.4: Average Run-times in Seconds and Number of Features for Different Stages of Our Pipeline on Symmetry Dataset

\begin{tabular}{|c|c|c|c|}
\hline General Steps & - & Ensemble SIFT & - \\
\hline MSER Neighborhood Assignment & 8.188724 & SIFT Feature Detection & 0.403183 \\
\hline LSD Extraction & 0.827009 & Number of SIFT Features & 4574 \\
\hline Number of Lines & 1128 & SIFT Feature Matching & 1.104771 \\
\hline Individual Linear Descriptor Construction & 0.003683 & Pair-wise Linear Descriptor Comparison & 0.000004 \\
\hline Individual HOG Descriptor Construction & 0.004568 & Pair-wise HOG Descriptor Comparison & 0.004069 \\
\hline ICP Optimization & 447.459381 & Total Ensemble SIFT Matching & 54.007973 \\
\hline LM Optimization & 34.251392 & Homography Estimation & 0.021649 \\
\hline Contextual Harris-Laplacian & - & Contextual Pseudo Corners & - \\
\hline Harris-Laplacian Corner Extraction & 2.413574 & Pseudo Corner Extraction & 0.826131 \\
\hline Number of Harris-Laplacian Corners & 1310 & Number of Pseudo Corners & 625 \\
\hline HOG Descriptor Matching & 2.381177 & HOG Descriptor Matching & 0.450085 \\
\hline Linear Descriptor Matching & 31.772003 & Linear Descriptor Matching & 6.271157 \\
\hline Total HOG Contextual Harris-Laplacian Corner Matching & 6.870990 & Total HOG Contextual Pseudo Corner Matching & 2.647096 \\
\hline Total Linear Contextual Harris-Laplacian Corner Matching & 76.265503 & Total Linear Contextual Pseudo Corner Matching & 28.250336 \\
\hline Homography Estimation & 0.072110 & Homography Estimation & 0.067457 \\
\hline
\end{tabular}

three versions of our contextual work. To extract Harris-Corners, we use the VLFeat library [122]. We have also included the runtimes of publicly available code for several of the methods we compare our work to throughout this section in the supplementary material.

Table A.5: Average Runtimes in Seconds and Number of Features for Other Methods on Symmetry Dataset

\begin{tabular}{r|c} 
SIFTFlow & - \\
\hline Dense SIFT & 0.250315 \\
SIFT Flow Matching & 18.869501 \\
\hline \hline Symmetry & - \\
\hline Number of Intensity-Based Features & 6.924392 \\
Gradient-Based Detector & 14.648718 \\
Number Gradient-Based of Features & 309 \\
Symmetry Descriptor Constructor & 11.240939
\end{tabular}


Table ?? shows runtimes and the number of features found for individual image pairs in the Symmetry dataset. This is a more detailed version of the average runtimes presented in the above table and correspond to the same set of tests. Table A.5 shows the average runtimes for the same set of images using publicly available code for some of the methods we compared our work to in the evaluation section of the main paper. 


\section{A.5 Supplemental Quantitative Registration Re-}

sults 


\section{Keypoint Matching Measurements}

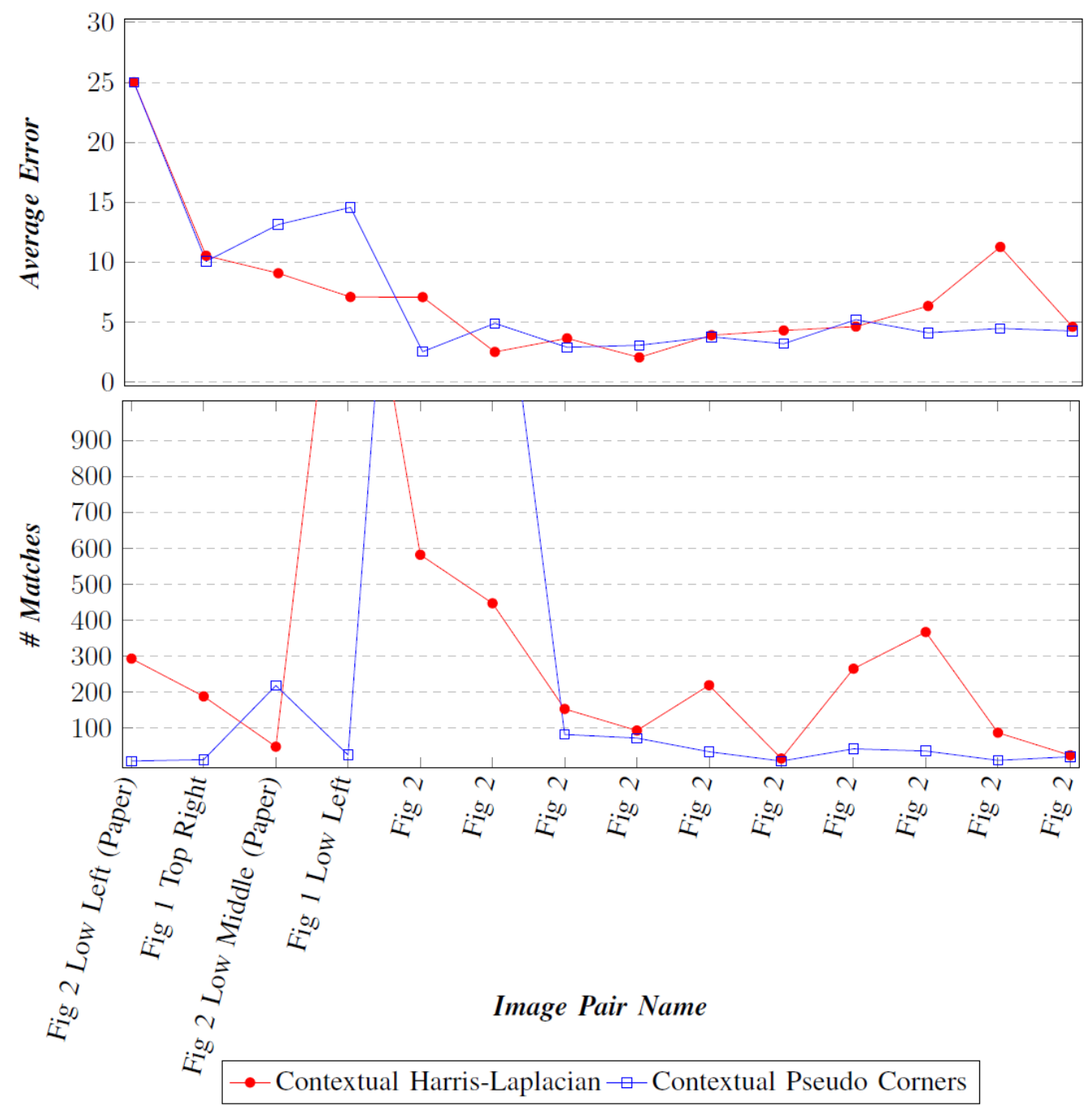

Figure A.6: Image pair alignment accuracy and number of matches found using Contextual Harris-Laplacian and Contextual Pseudo Corners on various datasets. The results for Figure 13 correspond to the images shown in Figure 13 in the submitted paper from left to right and come from the dataset provided by [1]. The results for Figure A.2 correspond to the images in Figure A.2 in this document from left to right and from top to bottom. These images come from the datasets provided by [1] and [8]. 
Keypoint Matching Measurements
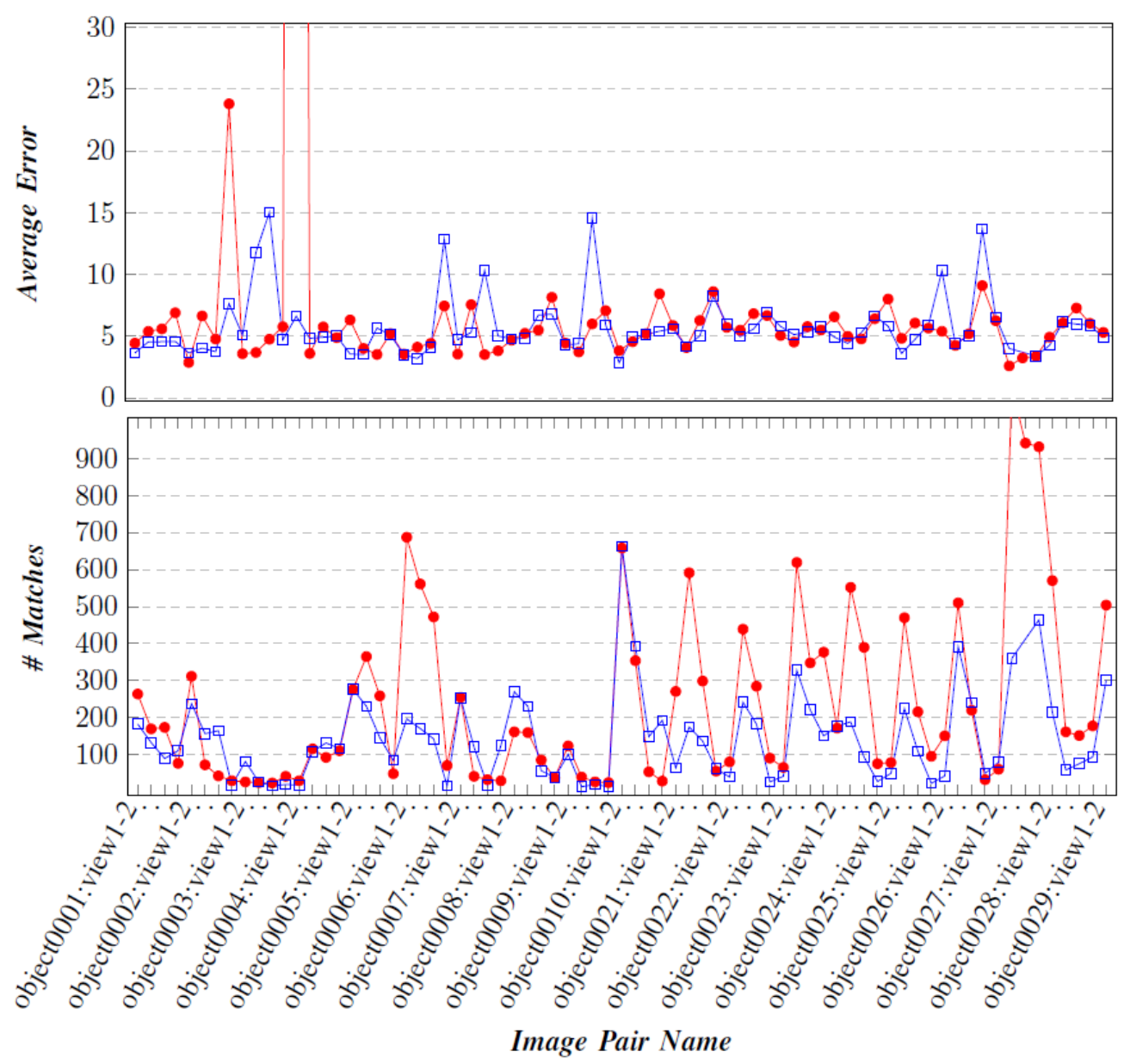

- - Contextual Harris-Laplacian $-\square$-Contextual Pseudo Corners

$\mathrm{m}$

Figure A.7: Image pair alignment accuracy and number of matches found using Contextual Harris-Laplacian and Contextual Pseudo Corners on images from the png$\mathrm{ZuBuD}$ dataset [6]. Images object0001-view1:2 through object0029-view1:5 are images of buildings in Zurich from various view points. Each building is assigned an object number and there are five views of each building. We matched view 1 to view 2, view 1 to view 3, and so for each building set for objects 1-10 and 21-29 in this dataset. 
Appendix B

Supplementary Material for 2D Matching Using Repetitive and Salient Features in Architectural Images 


\section{1-D Image Alignment Measurements}

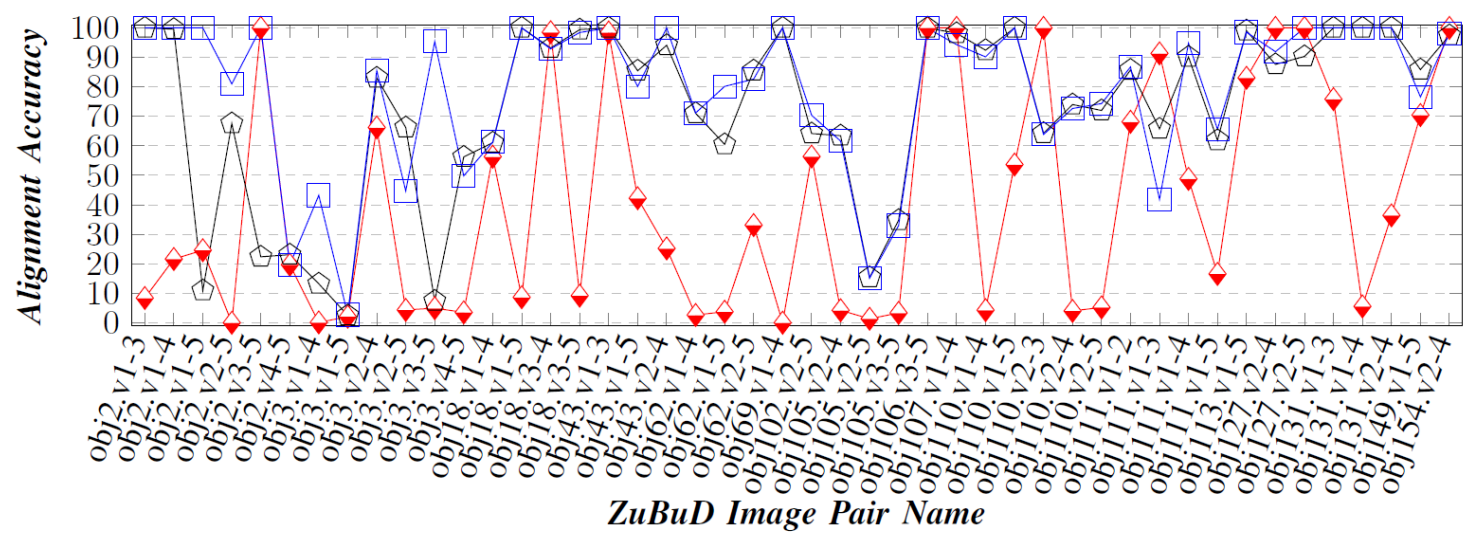

- Traditional SIFT $\triangle$ 1-D SIFT $\square$ 1-D Grouping

Figure B.1: Test of our proposed dimension reduction on the $\mathrm{ZuBuD}$ dataset. Here we compare the 1-dimensional accuracy (along the y-axis) of different methods. The first is to using traditional SIFT matches with the 8-point algorithm to compute a full 8 degree of freedom homography. The second approach is to use traditional SIFT matches, but compute a 1-D, 2 degree of freedom transformation using just the y-coordinates of the SIFT matches. The third approach is the grouping method described in this paper to compute a 1-D vertical transformation

1-D Image Alignment Measurements

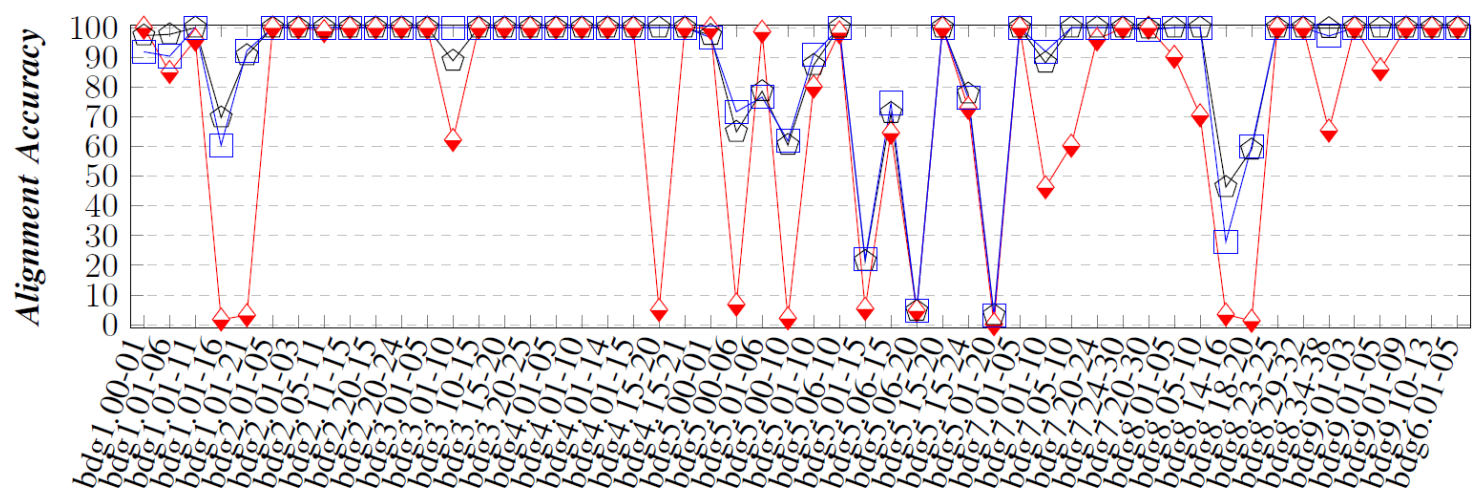

SymUrban Image Pair Name

$\triangleleft$ Traditional SIFT $\bullet$ 1-D SIFT $\square$ 1-D Grouping

Figure B.2: Test of our proposed dimension reduction. Here we compare the 1dimensional accuracy (along the y-axis) of different methods. The first is to using traditional SIFT matches with the 8-point algorithm to compute a full 8 degree of freedom homography. The second approach is to use traditional SIFT matches, but compute a 1-D, 2 degree of freedom transformation (as described in the main paper) using just the y-coordinates of the SIFT matches. The third approach is the grouping method described in this paper to compute a 1-D vertical transformation. 
1-D Image Alignment Measurements

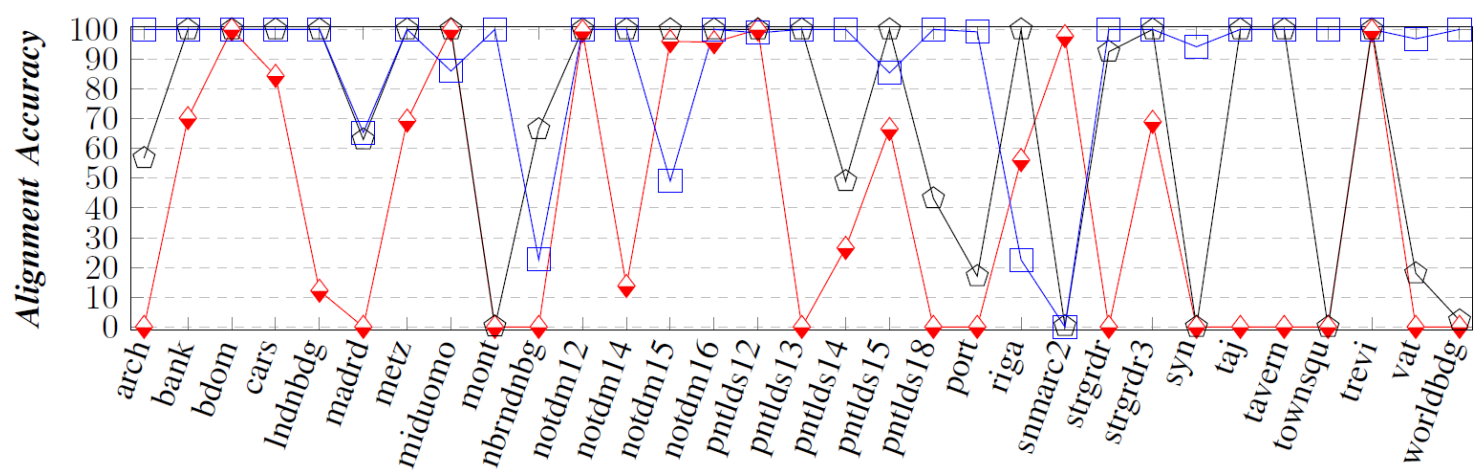

SymFeat Pair Name

$\diamond$ Traditional SIFT $\bullet$ 1-D SIFT $\square 1-\mathrm{D}$ Grouping

Figure B.3: Test of our proposed dimension reduction. Here we compare the 1dimensional accuracy (along the y-axis) of different methods. The first is to using traditional SIFT matches with the 8-point algorithm to compute a full 8 degree of freedom homography. The second approach is to use traditional SIFT matches, but compute a 1-D, 2 degree of freedom transformation (as described in the main paper) using just the y-coordinates of the SIFT matches. The third approach is the grouping method described in this paper to compute a 1-D vertical transformation. 
Two-Step Framework Alignment Measurements

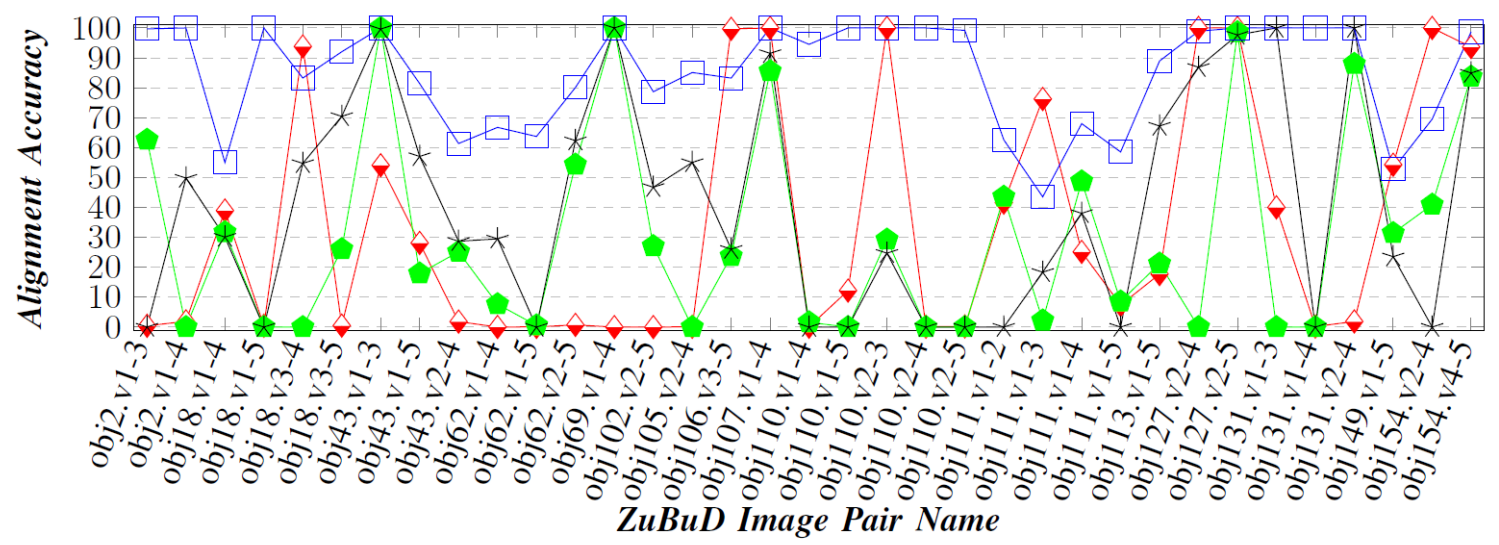

$\triangleleft$ Traditional SIFT - SIFT Two-Step $*$ Grouping Two-Step $\square$ Full Pipeline

Figure B.4: Test of our proposed two-step on the $\mathrm{ZuBuD}$ dataset. As a baseline, we show the accuracy of using traditional SIFT matching to compute a standard 8 DOF homography (Traditional SIFT). We then show tests for using 1) SIFT features (SIFT Two-Step) and 2) grouping features (Grouping Two-Step) to compute a 1-D vertical transformation. For both of these tests, we using matched SIFT features along corresponding rows to vote for a horizontal 1-D transformation. To show the strength of our full pipeline, we have also included in this chart the results of using the two-step approach with the intra-image saliency and pairwise match saliency computations (Full Pipeline). 
Two-Step Alignment Measurements

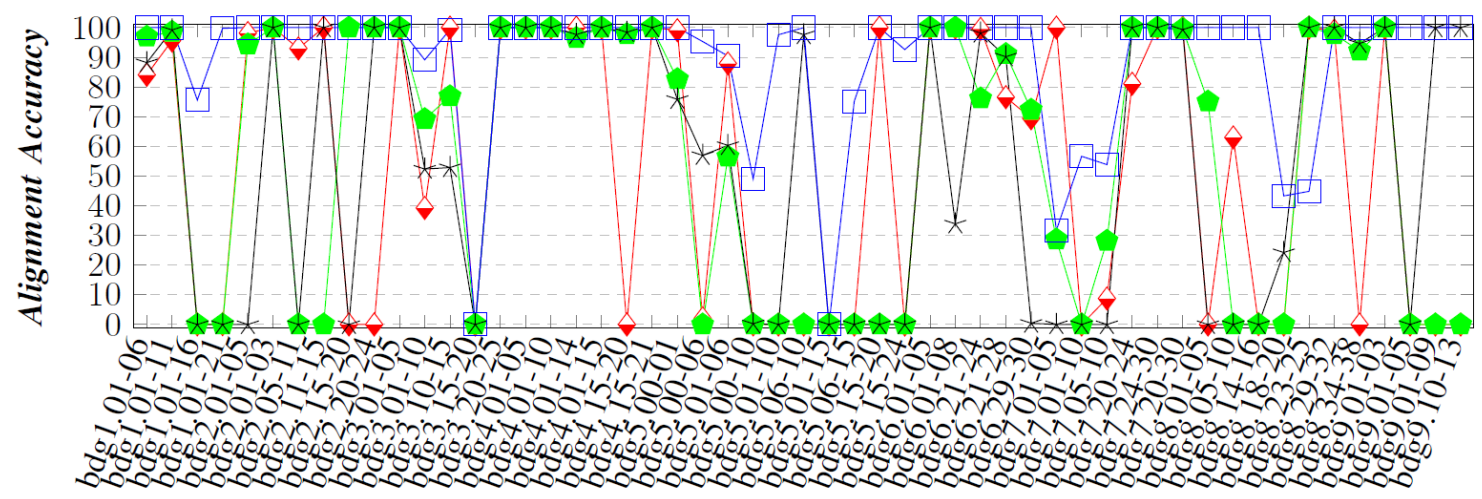

SymUrban Image Pair Name

- Traditional SIFT - SIFT Two-Step $*$ Grouping Two-Step $\square$ Full Pipeline

Figure B.5: Test of our proposed two-step on the SymUrban dataset. As a baseline, we show the accuracy of using traditional SIFT matching to compute a standard 8 DOF homography (Traditional SIFT). We then show tests for using 1) SIFT features (SIFT Two-Step) and 2) grouping features (Grouping Two-Step) to compute a 1-D vertical transformation. For both of these tests, we using matched SIFT features along corresponding rows to vote for a horizontal 1-D transformation. To show the strength of our full pipeline, we have also included in this chart the results of using the two-step approach with the intra-image saliency and pair-wise match saliency computations (Full Pipeline). 


\section{Bibliography}

[1] A. Shrivastava, T. Malisiewicz, A. Gupta, and A. A. Efros. Data-driven visual similarity for cross-domain image matching. In ACM Trans. on Graphics, volume 30, page 154. ACM, 2011.

[2] D. Hauagge and N. Snavely. Image matching using local symmetry features. In IEEE Comp. Vis. and Pattern Recongnit., pages 206-213. IEEE, 2012.

[3] K. Mikolajczyk, T. Tuytelaars, C. Schmid, A. Zisserman, J. Matas, F. Schaffalitzky, T. Kadir, and L. Van Gool. A comparison of affine region detectors. Int. Journ. of Comp. Vis., 65(1-2):43-72, 2005.

[4] B. Morago, G. Bui, and Y. Duan. An ensemble approach to image matching using contextual features. IEEE Trans. on Image Processing, 2015.

[5] D. Ceylan, N. J. Mitra, Y. Zheng, and M. Pauly. Coupled structure-frommotion and 3d symmetry detection for urban facades. Transactions on Graphics, 33(1):2, 2014.

[6] H. Shao, T. Svoboda, and L. Van Gool. Zubud-zurich buildings database for image based recognition. Comp. Vis. Lab, Swiss Federal Institute of Technology, Switzerland, Tech. Rep, 260, 2003. 
[7] G. Yang, C. V. Stewart, M. Sofka, and C. Tsai. Registration of challenging image pairs: Initialization, estimation, and decision. IEEE Trans. on Pattern Anal. and Mach. Intell., 29(11):1973-1989, 2007.

[8] V. R. Chandrasekhar, D. M. Chen, S. S. Tsai, N. Cheung, H. Chen, G. Takacs, Y. Reznik, R. Vedantham, R. Grzeszczuk, and J. Bach. The stanford mobile visual search data set. In ACM Multimedia systems, pages 117-122. ACM, 2011.

[9] C. Liu, J. Yuen, and A. Torralba. Sift flow: Dense correspondence across scenes and its applications. IEEE Pattern Anal. and Mach. Intell., 33(5):978-994, 2011.

[10] K. Mikolajczyk and C. Schmid. Scale \& affine invariant interest point detectors. Int. Journ. of Comp. Vis., 60(1):63-86, 2004.

[11] D. G. Lowe. Distinctive image features from scale-invariant keypoints. Int. Journ. of Comp. Vis., 60(2):91-110, 2004.

[12] M. A. Fischler and R. C. Bolles. Random sample consensus: a paradigm for model fitting with applications to image analysis and automated cartography. Communications of the ACM, 24(6):381-395, 1981.

[13] T. Tuytelaars and K. Mikolajczyk. Local invariant feature detectors: a survey. Foundations and Trends(R) in Comp. Graphics and Vis., 3(3):177-280, 2008.

[14] C. Harris and M. Stephens. A combined corner and edge detector. In Proc. Alvey Vis. Conf., volume 15, page 50. Manchester, UK, 1988.

[15] K. Mikolajczyk and C. Schmid. Indexing based on scale invariant interest points. In IEEE Proc. Int. Conf. on Comp. Vis., volume 1, pages 525-531. IEEE, 2001.

[16] J. Morel and G. Yu. Asift: A new framework for fully affine invariant image comparison. SIAM Journ. on Imaging Sciences, 2(2):438-469, 2009. 
[17] H. Bay, T. Tuytelaars, and L. Van Gool. Surf: Speeded up robust features. In Euro. Conf. on Comp. Vis., pages 404-417. Springer, 2006.

[18] C. Wu, F. Fraundorfer J.-M., Frahm, and M. Pollefeys. 3d model search and pose estimation from single images using vip features. In IEEE Comp. Vis. and Pattern Recongnit. Workshops, pages 1-8. IEEE, 2008.

[19] Y. HaCohen, E. Shechtman, D. B. Goldman, and D. Lischinski. Non-rigid dense correspondence with applications for image enhancement. In ACM ACM Trans. on Graphics, volume 30, page 70. ACM, 2011.

[20] P. Forssen and D. G. Lowe. Shape descriptors for maximally stable extremal regions. In IEEE Int. Conf. on Comp. Vis., pages 1-8. IEEE, 2007.

[21] R. Kimmel, C. Zhang, A. M. Bronstein, and M. M. Bronstein. Are mser features really interesting? IEEE Trans. on Pattern Anal. and Mach. Intell., 33(11):2316-2320, 2011.

[22] S. Belongie, J. Malik, and J. Puzicha. Shape matching and object recognition using shape contexts. IEEE Trans. on Pattern Anal. and Mach. Intell., 24(4):509-522, 2002.

[23] Richard Szeliski. Image alignment and stitching: A tutorial. Foundations and Trends@ in Comp. Graphics and Vis., 2(1):1-104, 2006.

[24] N. Dalal and B. Triggs. Histograms of oriented gradients for human detection. In IEEE Comp. Vis. and Pattern Recongnit., volume 1, pages 886-893. IEEE, 2005.

[25] Grant Schindler, Panchapagesan Krishnamurthy, Roberto Lublinerman, Yanxi Liu, and Frank Dellaert. Detecting and matching repeated patterns for au- 
tomatic geo-tagging in urban environments. In Computer Vision and Pattern Recognition, 2008. CVPR 2008. IEEE Conference on, pages 1-7. IEEE, 2008.

[26] E. Shechtman and M. Irani. Matching local self-similarities across images and videos. In IEEE Comp. Vis. and Pattern Recongnit., pages 1-8. IEEE, 2007.

[27] S. Singh and A. Guptaand A. A. Efros. Unsupervised discovery of mid-level discriminative patches. In Euro. Conf. on Comp. Vis., pages 73-86. Springer, 2012 .

[28] M. Aubry, B. C. Russell, and J. Sivic. Painting-to-3d model alignment via discriminative visual elements. ACM Trans. on Graphics, 33(2):14, 2014.

[29] B. C. Russell, J. Sivic, J. Ponce, and H. Dessales. Automatic alignment of paintings and photographs depicting a $3 \mathrm{~d}$ scene. In IEEE Int. Conf. on Comp. Vis. Workshops, pages 545-552. IEEE, 2011.

[30] L. Wang, U. Neumann, and S. You. Wide-baseline image matching using line signatures. In IEEE Int. Conf. on Comp. Vis., pages 1311-1318. IEEE, 2009.

[31] B. Fan, F. Wu, and Z. Hu. Line matching leveraged by point correspondences. In IEEE Comp. Vis. and Pattern Recongnit., pages 390-397. IEEE, 2010.

[32] H. Bay, V. Ferrari, and L. Van Gool. Wide-baseline stereo matching with line segments. In IEEE Comp. Vis. and Pattern Recongnit., volume 1, pages 329336. IEEE, 2005.

[33] J. Matas, O. Chum, M. Urban, and T. Pajdla. Distinguished regions for widebaseline stereo. Center for Machine Perception-K333 CVUT, Praha, Tech. Rep., 2001.

[34] V. Gioi, R. Grompone, J. Jakubowicz, J. Morel, and G. Randall. Lsd: a line segment detector. Image Processing 132 L Line, 2012. 
[35] J. Sivic, B. C. Russell, A. A. Efros, A. Zisserman, and W. T. Freeman. Discovering objects and their location in images. In IEEE Int. Conf. on Comp. Vis., volume 1, pages 370-377. IEEE, 2005.

[36] Z. Wu, Q. Ke, M. Isard, and J. Sun. Bundling features for large scale partialduplicate web image search. In IEEE Comp. Vis. and Pattern Recongnit., pages 25-32. IEEE, 2009.

[37] Cyrill Stachniss. C implementation of the hungarian method. Last accessed October, 27:2015, 2004.

[38] R. Hartley and A. Zisserman. Multiple View Geometry. Cambridge University Press, Cambridge, United Kingdom, 2010.

[39] T. Vincent and R. Laganiére. Detecting planar homographies in an image pair. In Proc. of Image and Signal Processing and Anal., pages 182-187. IEEE, 2001.

[40] Friedrich Fraundorfer, Konrad Schindler, and Horst Bischof. Piecewise planar scene reconstruction from sparse correspondences. Image and vision computing, 24(4):395-406, 2006.

[41] D. Santosh Kumar and C. V. Jawahar. Robust homography-based control for camera positioning in piecewise planar environments. In Comp. Vis., Graphics and Image Processing, pages 906-918. Springer, 2006.

[42] W. Lin, S. Liu, Y. Matsushita, T. Ng, and L. Cheong. Smoothly varying affine stitching. In IEEE Comp. Vis. and Pattern Recongnit., pages 345-352. IEEE, 2011.

[43] J. Zaragoza, T. Chin, M. S. Brown, and D. Suter. As-projective-as-possible image stitching with moving dlt. In IEEE Comp. Vis. and Pattern Recongnit., pages 2339-2346. IEEE, 2013. 
[44] Y. Lipman, S. Yagev, R. Poranne, D. W. Jacobs, and R. Basri. Feature matching with bounded distortion. ACM Trans. on Graphics, 33(3):26, 2014.

[45] W. D. Lin, M. Cheng, J. Lu, H. Yang, M. N. Do, and P. Torr. Bilateral functions for global motion modeling. In Euro. Conf. on Comp. Vis., pages 341-356. Springer, 2014.

[46] H. Yang, W. Lin, and J. Lu. Daisy filter flow: a generalized discrete approach to dense correspondences. In IEEE Comp. Vis. and Pattern Recongnit., pages 3406-3413. IEEE, 2014.

[47] P. Besl and N. McKay. Method for registration of 3-d shapes. In Proc. SPIE Sensor Fusion IV: Control Paradigms and Data Structures, pages 586-606. Int. Society for Optics and Photonics, 1992.

[48] N. Snavely, S.M. Seitz, and R. Szeliski. Modeling the world from internet photo collections. Int. Journ. of Comp. Vis., 80(2):189-210, 2007.

[49] M. Park, S. Lee, P. Chen, S. Kashyap, A. Butt, and Y. Liu. Performance evaluation of state-of-the-art discrete symmetry detection algorithms. In Computer Vision and Pattern Recognition, pages 1-8. IEEE, 2008.

[50] C. Wu, J. Frahm, and M. Pollefeys. Detecting large repetitive structures with salient boundaries. In European Conference on Computer Vision, pages 142155. Springer, 2010.

[51] M. Kushnir and I. Shimshoni. Epipolar geometry estimation for urban scenes with repetitive structures. IEEE Transactions on Pattern Analysis and Machine Intelligence, 36(12):2381-2395, 2014. 
[52] Y. Chung, T. Han, and Z. He. Building recognition using sketch-based representations and spectral graph matching. In IEEE 12th International Conference on Computer Vision, pages 2014-2020, 2009.

[53] M. Bansal, K. Daniilidis, and H. Sawhney. Ultra-wide baseline facade matching for geo-localization. In European Conference on Computer Vision Workshops and Demonstrations, pages 175-186. Springer, 2012.

[54] C. Barnes, E. Shechtman, A. Finkelstein, and D. Goldman. Patchmatch: A randomized correspondence algorithm for structural image editing. ACM Transactions on Graphics, 28(3):24, 2009.

[55] C. Barnes, E. Shechtman, and D. Goldman. The generalized patchmatch correspondence algorithm. pages 29-43. Springer, 2010.

[56] S. Singh, A. Gupta, and A. Efros. Unsupervised discovery of mid-level discriminative patches. European Conference on Computer Vision, pages 73-86, 2012.

[57] N. Otsu. A threshold selection method from gray-level histograms. Automatica, 11(285-296):23-27, 1975.

[58] J. Lezama, R. Grompone von Gioi, G. Randall, and J. Morel. Finding vanishing points via point alignments in image primal and dual domains. In Computer Vision and Pattern Recognition, pages 509-515. IEEE, 2014.

[59] N. Snavely, S.M. Seitz, and R. Szeliski. Modeling the world from internet photo collections. International Journal of Computer Vision, 80(2):189-210, 2008.

[60] Jiwon Kim, Jung Kwon Lee, and Kyoung Mu Lee. Accurate image super-resolution using very deep convolutional networks. arXiv preprint arXiv:1511.04587, 2015. 
[61] Hong Chang, Dit-Yan Yeung, and Yimin Xiong. Super-resolution through neighbor embedding. In Computer Vision and Pattern Recognition, 2004. CVPR 2004. Proceedings of the 2004 IEEE Computer Society Conference on, volume 1, pages I-I. IEEE, 2004.

[62] Saining Xie and Zhuowen Tu. Holistically-nested edge detection. In Proceedings of the IEEE International Conference on Computer Vision, pages 1395-1403, 2015.

[63] Chen-Yu Lee, Saining Xie, Patrick Gallagher, Zhengyou Zhang, and Zhuowen Tu. Deeply-supervised nets. In Artificial Intelligence and Statistics, pages 562570,2015 .

[64] Chih-Yuan Yang, Chao Ma, and Ming-Hsuan Yang. Single-image superresolution: A benchmark. In European Conference on Computer Vision, pages 372-386. Springer, 2014.

[65] Simon Baker and Takeo Kanade. Limits on super-resolution and how to break them. IEEE Transactions on Pattern Analysis and Machine Intelligence, 24(9):1167-1183, 2002.

[66] Michal Irani and Shmuel Peleg. Improving resolution by image registration. CVGIP: Graphical models and image processing, 53(3):231-239, 1991.

[67] Shengyang Dai, Mei Han, Wei Xu, Ying Wu, and Yihong Gong. Soft edge smoothness prior for alpha channel super resolution. In Computer Vision and Pattern Recognition, 200\%. CVPR'0\%. IEEE Conference on, pages 1-8. IEEE, 2007.

[68] Jian Sun, Zongben Xu, and Heung-Yeung Shum. Image super-resolution using gradient profile prior. In Computer Vision and Pattern Recognition, 2008. CVPR 2008. IEEE Conference on, 136 ges 1-8. IEEE, 2008. 
[69] Qi Shan, Zhaorong Li, Jiaya Jia, and Chi-Keung Tang. Fast image/video upsampling. ACM Transactions on Graphics (TOG), 27(5):153, 2008.

[70] Jinggang Huang and David Mumford. Statistics of natural images and models. In Computer Vision and Pattern Recognition, 1999. IEEE Computer Society Conference On., volume 1, pages 541-547. IEEE, 1999.

[71] Kwang In Kim and Younghee Kwon. Single-image super-resolution using sparse regression and natural image prior. IEEE transactions on pattern analysis and machine intelligence, 32(6):1127-1133, 2010.

[72] Haichao Zhang, Jianchao Yang, Yanning Zhang, and Thomas Huang. Non-local kernel regression for image and video restoration. Computer Vision-ECCV 2010, pages 566-579, 2010.

[73] D Dai, R Timofte, and L Van Gool. Jointly optimized regressors for image super-resolution. In Computer Graphics Forum, volume 34, pages 95-104. Wiley Online Library, 2015.

[74] Jianchao Yang, John Wright, Thomas Huang, and Yi Ma. Image superresolution as sparse representation of raw image patches. In Computer Vision and Pattern Recognition, 2008. CVPR 2008. IEEE Conference on, pages 1-8. IEEE, 2008.

[75] Jianchao Yang, John Wright, Thomas S. Huang, and Yi Ma. Image superresolution via sparse representation. IEEE Transactions on Image Processing, 19(11):2861-2873, 112010.

[76] Zhen Cui, Hong Chang, Shiguang Shan, Bineng Zhong, and Xilin Chen. Deep network cascade for image super-resolution. In European Conference on Computer Vision, pages 49-64. Springer, 2014. 
[77] Gilad Freedman and Raanan Fattal. Image and video upscaling from local self-examples. ACM Transactions on Graphics (TOG), 30(2):12, 2011.

[78] Daniel Glasner, Shai Bagon, and Michal Irani. Super-resolution from a single image. In 2009 IEEE 12th International Conference on Computer Vision, pages 349-356. IEEE, 2009.

[79] Jia-Bin Huang, Abhishek Singh, and Narendra Ahuja. Single image superresolution from transformed self-exemplars. In 2015 IEEE Conference on Computer Vision and Pattern Recognition (CVPR), pages 5197-5206. IEEE, 2015.

[80] Chih-Yuan Yang, Jia-Bin Huang, and Ming-Hsuan Yang. Exploiting selfsimilarities for single frame super-resolution. In Proceedings of the Asian Conference on Computer Vision, pages 497-510, 2011.

[81] Kui Jia, Xiaogang Wang, and Xiaoou Tang. Image transformation based on learning dictionaries across image spaces. IEEE transactions on pattern analysis and machine intelligence, 35(2):367-380, 2013.

[82] Samuel Schulter, Christian Leistner, and Horst Bischof. Fast and accurate image upscaling with super-resolution forests. In Proceedings of the IEEE Conference on Computer Vision and Pattern Recognition, pages 3791-3799, 2015.

[83] Radu Timofte, Vincent De Smet, and Luc Van Gool. Anchored neighborhood regression for fast example-based super-resolution. In Proceedings of the IEEE International Conference on Computer Vision, pages 1920-1927, 2013.

[84] Radu Timofte, Vincent De Smet, and Luc Van Gool. A+: Adjusted anchored neighborhood regression for fast super-resolution. In Asian Conference on Computer Vision, pages 111-126. Springer, 2014. 
[85] Jianchao Yang, Zhaowen Wang, Zhe Lin, Scott Cohen, and Thomas Huang. Coupled dictionary training for image super-resolution. IEEE Transactions on Image Processing, 21(8):3467-3478, 2012.

[86] Chao Dong, Chen Change Loy, Kaiming He, and Xiaoou Tang. Image superresolution using deep convolutional networks. IEEE Transactions on Pattern Analysis and Machine Intelligence, pages 295-307, 52015.

[87] Zhaowen Wang, Ding Liu, Jianchao Yang, Wei Han, and Thomas Huang. Deep networks for image super-resolution with sparse prior. In Proceedings of the IEEE International Conference on Computer Vision, pages 370-378, 2015.

[88] Jiwon Kim, Jung Kwon Lee, and Kyoung Mu Lee. Deeply-recursive convolutional network for image super-resolution. arXiv preprint arXiv:1511.04491, 2015.

[89] Wenzhe Shi, Jose Caballero, Ferenc Huszár, Johannes Totz, Andrew P Aitken, Rob Bishop, Daniel Rueckert, and Zehan Wang. Real-time single image and video super-resolution using an efficient sub-pixel convolutional neural network. In Proceedings of the IEEE Conference on Computer Vision and Pattern Recognition, pages 1874-1883, 2016.

[90] Chao Dong, Chen Change Loy, and Xiaoou Tang. Accelerating the superresolution convolutional neural network. In European Conference on Computer Vision, pages 391-407. Springer, 2016.

[91] David Martin, Charless Fowlkes, Doron Tal, and Jitendra Malik. A database of human segmented natural images and its application to evaluating segmentation algorithms and measuring ecological statistics. In Computer Vision, 2001. ICCV 2001. Proceedings. Eighth IEEE International Conference on, volume 2, pages 416-423. IEEE, 2001. 
[92] Marco Bevilacqua, Aline Roumy, Christine Guillemot, and Marie Line AlberiMorel. Low-complexity single-image super-resolution based on nonnegative neighbor embedding. 2012.

[93] Roman Zeyde, Michael Elad, and Matan Protter. On single image scale-up using sparse-representations. In International conference on curves and surfaces, pages 711-730. Springer, 2010.

[94] Radu Timofte, Rasmus Rothe, and Luc Van Gool. Seven ways to improve example-based single image super resolution. In Proceedings of the IEEE Conference on Computer Vision and Pattern Recognition, pages 1865-1873, 2016.

[95] Sander Dieleman, Jan Schluter, Colin Raffel, Eben Olson, Soren Kaae Sonderby, Daniel Nouri, Daniel Maturana, Martin Thoma, Eric Battenberg, Jack Kelly, Jeffrey De Fauw, Michael Heilman, Diogo Moitinho de Almeida, Brian McFee, Hendrik Weideman, Gabor Takacs, Peter de Rivaz, Jon Crall, Gregory Sanders, Kashif Rasul, Cong Liu, Geoffrey French, and Jonas Degrave. Lasagne: First release, 82015 .

[96] James Bergstra, Olivier Breuleux, Frederic Bastien, Pascal Lamblin, Razvan Pascanu, Guillaume Desjardins, Joseph Turian, David Warde-Farley, and Yoshua Bengio. Theano: a cpu and gpu math expression compiler. In Scientific Computing with Python Conference, 62010.

[97] Diederik Kingma and Jimmy Ba. Adam: A method for stochastic optimization. arXiv preprint arXiv:1412.6980, 2014.

[98] Matthias Zwicker, Hanspeter Pfister, Jeroen Van Baar, and Markus Gross. Surface splatting. In Proceedings of the 28th annual conference on Computer graphics and interactive techniques, pages 371-378. ACM, 2001. 
[99] Mario Botsch, Alexander Hornung, Matthias Zwicker, and Leif Kobbelt. Highquality surface splatting on today's gpus. In Proceedings Eurographics/IEEE VGTC Symposium Point-Based Graphics, 2005., pages 17-141. IEEE, 2005.

[100] Dominik Sibbing, Torsten Sattler, Bastian Leibe, and Leif Kobbelt. Sift-realistic rendering. In International Conference on 3D Vision, pages 56-63, 72013.

[101] Christian Ledig, Lucas Theis, Ferenc Huszár, Jose Caballero, Andrew Cunningham, Alejandro Acosta, Andrew Aitken, Alykhan Tejani, Johannes Totz, Zehan Wang, et al. Photo-realistic single image super-resolution using a generative adversarial network. arXiv preprint, 2016.

[102] Kaiming He, Xiangyu Zhang, Shaoqing Ren, and Jian Sun. Deep residual learning for image recognition. In Proceedings of the IEEE conference on computer vision and pattern recognition, pages 770-778, 2016.

[103] Bee Lim, Sanghyun Son, Heewon Kim, Seungjun Nah, and Kyoung Mu Lee. Enhanced deep residual networks for single image super-resolution. In Computer Vision and Pattern Recognition Workshops (CVPRW), 2017 IEEE Conference on, pages 1132-1140. IEEE, 2017.

[104] Radu Timofte, Eirikur Agustsson, Luc Van Gool, Ming-Hsuan Yang, Lei Zhang, Bee Lim, Sanghyun Son, Heewon Kim, Seungjun Nah, Kyoung Mu Lee, et al. Ntire 2017 challenge on single image super-resolution: Methods and results. In Computer Vision and Pattern Recognition Workshops (CVPRW), 2017 IEEE Conference on, pages 1110-1121. IEEE, 2017.

[105] Ian Goodfellow, Jean Pouget-Abadie, Mehdi Mirza, Bing Xu, David WardeFarley, Sherjil Ozair, Aaron Courville, and Yoshua Bengio. Generative adversarial nets. In Advances in neural information processing systems, pages 2672-2680, 2014. 
[106] Michael Mathieu, Camille Couprie, and Yann LeCun. Deep multi-scale video prediction beyond mean square error. arXiv preprint arXiv:1511.05440, 2015.

[107] Emily L Denton, Soumith Chintala, Rob Fergus, et al. Deep generative image models using a laplacian pyramid of adversarial networks. In Advances in neural information processing systems, pages 1486-1494, 2015.

[108] Alec Radford, Luke Metz, and Soumith Chintala. Unsupervised representation learning with deep convolutional generative adversarial networks. arXiv preprint arXiv:1511.06434, 2015.

[109] Justin Johnson, Alexandre Alahi, and Li Fei-Fei. Perceptual losses for realtime style transfer and super-resolution. In European Conference on Computer Vision, pages 694-711. Springer, 2016.

[110] K. Simonyan and A. Zisserman. Very deep convolutional networks for largescale image recognition. CoRR, abs/1409.1556, 2014.

[111] Noah Snavely, Steven M Seitz, and Richard Szeliski. Photo tourism: exploring photo collections in 3d. In ACM transactions on graphics (TOG), volume 25, pages 835-846. ACM, 2006.

[112] Yunpeng Li, Noah Snavely, and Daniel P Huttenlocher. Location recognition using prioritized feature matching. In European conference on computer vision, pages 791-804. Springer, 2010.

[113] Leif Kobbelt and Mario Botsch. A survey of point-based techniques in computer graphics. Computers $\& 3$ Graphics, 28(6):801-814, 2004.

[114] Arnold Irschara, Christopher Zach, Jan-Michael Frahm, and Horst Bischof. From structure-from-motion point clouds to fast location recognition. In Com- 
puter Vision and Pattern Recognition, 2009. CVPR 2009. IEEE Conference on, pages 2599-2606. IEEE, 2009.

[115] Martın Abadi, Ashish Agarwal, Paul Barham, Eugene Brevdo, Zhifeng Chen, Craig Citro, Greg S Corrado, Andy Davis, Jeffrey Dean, Matthieu Devin, et al. Tensorflow: Large-scale machine learning on heterogeneous systems, 2015. url $\mathrm{h}$ ttp. tensorflow. org/. So ftware available from tensorflow. org.

[116] David G Lowe. Distinctive image features from scale-invariant keypoints. International journal of computer vision, 60(2):91-110, 2004.

[117] L. Moisan, P. Moulon, and P. Monasse. Automatic homographic registration of a pair of images, with a contrario elimination of outliers. Image Processing On Line, 10, 2012.

[118] J. Philbin, O. Chum, M. Isard, J. Sivic, and A. Zisserman. Object retrieval with large vocabularies and fast spatial matching. In Comp. Vis. and Pattern Recongnit. IEEE, 2007.

[119] H. Shao, T. Svoboda, and L. Van Gool. Zubud-zurich buildings database for image based recognition. Comp. Vis. Lab, Swiss Federal Institute of Technology, Switzerland, Tech. Rep, 260, 2003.

[120] J. Matas, O. Chum, M. Urban, and T. Pajdla. Robust wide-baseline stereo from maximally stable extremal regions. Image and vision computing, 22(10):761$767,2004$.

[121] G. Bradski. Opencv library. Dr. Dobb's Journ. of Software Tools, 2000.

[122] Andrea Vedaldi and Brian Fulkerson. Vlfeat: An open and portable library of computer vision algorithms. In Proceedings of the 18th ACM international conference on Multimedia, pages 1469-1472. ACM, 2010. 


\section{VITA}

Giang Bui was born in Ha Tay, Vietnam. He attended the Hanoi National University where she graduated with a B.S. Degree in Applied Mathematics and Informatics in 2004. He also got a M.S Degree in Information Technology in 2008. Hw will be completing her Ph.D. in Computer Science at the University of Missouri. In 2011, Giang began working with Ye Duan on computer vision-related research with his main project $2 \mathrm{D}$ image matching and image super-resolution via deep-learning. 